\title{
Light trapping in thin-film silicon solar cells via plasmonic metal nanoparticles
}

\author{
by \\ Ryan J. Veenkamp, B.Sc. Engineering \\ A thesis submitted to the \\ Faculty of Graduate and Postdoctoral Affairs \\ in partial fulfillment of the requirements for the degree of
}

Master of Applied Science in Electrical and Computer Engineering

Ottawa-Carleton Institute for Electrical and Computer Engineering

Department of Electronics

Carleton University

Ottawa, Ontario

September, 2014

(C)Copyright

Ryan J. Veenkamp, 2014 
The undersigned hereby recommends to the

Faculty of Graduate and Postdoctoral Affairs

acceptance of the thesis

\title{
Light trapping in thin-film silicon solar cells via plasmonic metal nanoparticles
}

\author{
submitted by Ryan J. Veenkamp, B.Sc. Engineering \\ in partial fulfillment of the requirements for the degree of
}

Master of Applied Science in Electrical and Computer Engineering

Professor Winnie N. Ye, Thesis Supervisor

Professor Niall Tait, Chair,

Department of Electrical and Computer Engineering

Ottawa-Carleton Institute for Electrical and Computer Engineering

Department of Electronics

Carleton University

September, 2014 


\section{Abstract}

Cost cutting for solar cells in grid power applications is necessary in order to drive large-scale adoption and use of renewable solar energy. Despite the large recent cost reductions in crystalline silicon solar cells, thin-film solar cells also have a viable place in the market. These cells have several potential advantages over their thicker bulk crystalline silicon cousins including lower embodied energy and material costs, higher optical absorption rates and lower material purity requirements. The continuing issue with thin-film solar cells is the short optical path length through the absorbing layer and solving this whilst not increasing recombination rates requires the use of clever light trapping techniques. The purpose of this thesis is to investigate the effectiveness of plasmonic nanoparticles, in particular nanocubes, for light trapping in thin-film solar cells through numerical simulation and experimental demonstrations.

We first investigate the optical properties of plasmonic metal nanoparticles (MNPs) based on the potential for scattering incident solar radiation into an absorbing substrate at large angles, significantly increasing optical path length. MNPs are then optimized in terms of shape, size, material and dielectric environment, and their effect on short circuit current $\left(J_{s c}\right)$ enhancement is characterized. Al nanocubes are shown in simulation to obtain up to $40 \% J_{s c}$ enhancement and subsequently demonstrated on a thin-film nanocrystalline silicon solar cell to be the most viable option for light trapping. 


\section{Acknowledgments}

I would like to acknowledge the help and contributions of all the people who made this thesis possible, without you I would never have made it to this point. First to the Carleton Microfabrication Lab technicians Rob Vandusen, Rick Adams and Angela McCormick for their invaluable assistance and input in fabricating the solar cells as well as Dr. Garry Tarr for assistance in designing and fabricating the solar cell mask. I would like to thank my collaborator Mohsen Mahmoudysepehr at the University of Waterloo who deposited the nanocrystalline silicon layers which made the experiment possible. Also the knowledge and e-beam lithography expertise for nanocube formation from Sebastian Schultz at the University of Ottawa. Testing would not have been possible without Ross Cheriton at SUNLAB, thank you for your help and input. Finally, I would like to express my sincere thanks to my supervisor, Dr. Winnie Ye, for her advice, motivation and many hours spent revising and editing my papers and eventually this thesis, your help was much appreciated. 


\section{Table of Contents}

Abstract $\quad$ iii

Acknowledgments $\quad$ iv

Table of Contents $\quad$ v

List of Tables $\quad$ vii

List of Figures viii

Nomenclature $\quad$ xiii

1 Introduction 1

1.1 Thesis Objectives . . . . . . . . . . . . . . . . . . 7

2 Literature Review $\quad 10$

3 Theory and Concepts 16

3.1 Thin-film a-Si solar cells . . . . . . . . . . . . . . . . . . 16

3.2 Plasmonics . . . . . . . . . . . . . . . . . . . . . 21

3.2.1 Mie Theory: An Analytical Solution to Maxwell's Equations for Spherical Particles . . . . . . . . . . . . . . . . . . 21

3.2.2 Surface Plasmons . . . . . . . . . . . . . . . . . . 27 
4.1 Simulation Methodology . . . . . . . . . . . . . . 37

4.2 Effect of MNP geometry on plasmonic enhancement . . . . . . . . . . 41

4.3 Effect of a dielectric spacer layer on MNP performance . . . . . . . . 44

4.4 Effect of MNP material on plasmonic enhancement . . . . . . . . 49

4.5 MNP Optimizations . . . . . . . . . . . . . . . . 57

4.6 Investigation of an optimal ITO spacer layer . . . . . . . . . . . . 63

4.7 Detailed Examination of Nanocube Behaviour . . . . . . . . . . 66

4.7.1 Comparison to Traditional Anti-reflection Coatings . . . . . . 66

4.7.2 Particle scattering and field enhancement . . . . . . . . . . 72

$\begin{array}{lll}5 & \text { Experimental Demonstration } & 76\end{array}$

5.1 Fabrication Methods . . . . . . . . . . . . . . 76

5.2 Testing Results . . . . . . . . . . . . . . . . . . . 83

$\begin{array}{lll}6 & \text { Conclusions } & 94\end{array}$

6.1 Future Work . . . . . . . . . . . . . . . . . 97

6.2 Achievements and Publications . . . . . . . . . . . . . . . 99

$\begin{array}{ll}\text { List of References } & 100\end{array}$

Appendix A Lumerical Post-processing Scripts 106

A.1 Scattering and Absorption Cross-sections (FDTD) . . . . . . . . 106

A.2 Data analysis using solar spectrum data (FDTD) . . . . . . . . . 109

A.3 I-V curve Calculation (DEVICE) . . . . . . . . . . . . . . 111

Appendix B Fabrication Process Flow Sheet 112 


\section{List of Tables}

1.1 External parameters of the best laboratory c-Si and a-Si solar cells [1] 5

3.1 Comparison of c-Si and a-Si $[1] \ldots \ldots \ldots$. . . . . . . . . . 17

4.1 Optimal parameters for $J_{s c}$ enhancement in a-Si solar cells . . . . . . 62

4.2 Best achievable $J_{s c}$ showing increase over cell without MNPs . . . . . 70

5.1 Parameters used for deposition of Si and ITO . . . . . . . . . 77 


\section{List of Figures}

1.1 Absorption coefficients of common solar cell absorber materials in the solar spectrum . . . . . . . . . . . . . . . . . . . 4

3.1 Representation of a-Si atomic structure [1] . . . . . . . . . . . . 18

3.2 Light scattering by particle of arbitrary shape . . . . . . . . . . . 22

3.3 Radiant flux through volume containing the entirety of the particle . 25

3.4 a) Interaction of a small metal nanoparticle with incident light. Also depicted is a particle exhibiting b) dipole radiation and c) quadrupole radiation for a larger particle . . . . . . . . . . . . .

3.5 Dielectric function and extinction cross-section of a) $\mathrm{Al}$ and b) $\mathrm{Ag}$

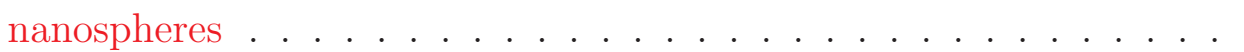

4.1 Unit cell geometry under investigation with a MNP (nanocube shown here) used as a scatterer on a thin film in contact with a semi-infinite substrate. . . . . . . . . . . . . . .

4.2 Normalized scattering cross sections of $150 \mathrm{~nm} \mathrm{Ag} \mathrm{cubes,} \mathrm{spheres} \mathrm{and}$ hemispheres sitting directly on an a-Si substrate. Inset shows MNP dimensions in $n m . \ldots \ldots \ldots \ldots \ldots$ 
4.3 Fraction of light scattered backwards $\left(1-F_{\text {subs }}\right)$ for $150 \mathrm{~nm}$ Ag cubes sitting directly on $n_{\text {subs( } \mathrm{P} 3 \mathrm{HT}: \mathrm{PCBM})}=1.8$ (dashes) and $n_{\mathrm{subs}(\mathrm{a}-\mathrm{Si})}=4.23$ (solid). Also shown, 150nm Ag spheres on P3HT:PCBM (dots) and a-Si (long dashes). Inset shows electric field distribution of the dipolar (i) and quadrupolar (ii) modes of a nanocube and the dipolar (i) mode of a nanosphere directly on a-Si. . . . . . . . . . . . . .

4.4 Extinction efficiency in 80 $\mathrm{nm}$ Ag nanocubes on substrates with increasing refractive indices $n_{\text {subs }}=1.8$ to $n_{\text {subs }}=4.23$. The dark blue curve reflects changes when a $25 \mathrm{~nm} \mathrm{SiO}_{2}$ spacer layer is inserted between the Ag nanocubes and an a-Si substrate. The resonant dipolar (i) modes are shown for each curve. . . . . . . . . . . . . . . . .

4.5 Optimization of $\mathrm{SiO}_{2}$ spacer layer thickness under a 25\% surface coverage of uniformly distributed $80 \mathrm{~nm} \mathrm{Ag}$ nanocubes in terms of $J_{s c}$ enhancement over an identical cell without MNPs, assuming that each photon absorbed creates an $e^{-} / h^{+}$pair contributing to the photocurrent. 47

4.6 Fraction of light scattered forward into the substrate $\left(F_{\text {subs }}\right)$ by $150 \mathrm{~nm}$ Ag MNPs and calibrated to the power available in the AM1.5G spectrum at each wavelength. The $\mathrm{SiO}_{2}$ spacer layer is set to the optimized size of $25 n m . \ldots \ldots \ldots \ldots \ldots \ldots$

4.7 (a) Normalized scattering cross-sections $\left(Q_{\text {scat }}\right)$. (b) Fraction of incident radiation forward scattered into the substrate $\left(F_{\text {subs }}\right)$. (c) Radiative efficiency of the particles $\left(N_{\text {rad }}\right)$. (d) Normalized absorption cross sections $\left(Q_{\mathrm{abs}}\right)$. The spacer thickness is set to $25 \mathrm{~nm}$ and all quantities are calibrated to the power available in the AM1.5G spectrum at each




4.8 Normalized scattering cross-section $\left(Q_{\text {scat }}\right)$ (calibrated to the power available in the AM1.5G spectrum) of $150 \mathrm{~nm}$ Al MNPs sitting directly on a-Si substrate or a $25 \mathrm{~nm} \mathrm{SiO}_{2}$ spacer layer. . . . . . . . . . . .

4.9 Power absorbed per $\mathrm{m}^{2} / \mathrm{nm}$ in a $240 \mathrm{~nm}$ a-Si layer with a $25 \%$ surface coverage of uniformly spaced 80nm nanoparticles on optimized $\mathrm{SiO}_{2}$ spacer layers . . . . . . . . . . . . . . . . .

4.10 Contour optimization of $80 \mathrm{~nm} \mathrm{Ag} \mathrm{a)} \mathrm{nanocube,} \mathrm{b)} \mathrm{nanohemisphere}$ and c) nanosphere surface coverage and $\mathrm{SIO}_{2}$ spacer layer thickness .

4.11 Contour optimization of 80nm $\mathrm{Ag}$ a) nanocube, b) nanohemisphere and c) nanosphere surface coverage and ITO spacer layer thickness . .

4.12 Contour optimization of $80 \mathrm{~nm} \mathrm{Al} \mathrm{a)} \mathrm{nanocube,} \mathrm{b)} \mathrm{nanohemisphere} \mathrm{and}$ c) nanosphere surface coverage and $\mathrm{SiO}_{2}$ spacer layer thickness . . . .

4.13 Contour optimization of $80 \mathrm{~nm} \mathrm{Al} \mathrm{a)} \mathrm{nanocube,} \mathrm{b)} \mathrm{nanohemisphere} \mathrm{and}$ c) nanosphere surface coverage and ITO spacer layer thickness . . . . 61

4.14 Normalized scattering cross-section $\left(Q_{\text {scat }}\right)$ compared to fraction of forward scattered light $\left(F_{\text {subs }}\right)$ for $80 \mathrm{~nm}$ a) $\mathrm{Ag}$ and b) Al nanocubes on a $25 n$ ITO spacer layer . . . . . . . . . . . . . . . . .

4.15 Power absorbed per $\mathrm{m}^{2} / \mathrm{nm}$ in a $240 \mathrm{~nm}$ a-Si solar cell with optimal $80 \mathrm{~nm}$ MNPs integrated on the surface of a thin $20 \mathrm{~nm}$ ITO top contact, compared with a particle free cell and the power available in the AM1.5G solar spectrum. . . . . . . . . . . . . . .

4.16 Intensity of scattered light from $80 \mathrm{~nm} \mathrm{Ag}$ cubes on $20 \mathrm{~nm}$ of a) $\mathrm{SiO}_{2}$, b) ITO and $80 \mathrm{~nm} \mathrm{Al} \mathrm{cubes} \mathrm{on} 20 \mathrm{~nm}$ of c) $\mathrm{SiO}_{2}$ and d) ITO at $500 \mathrm{~nm}$

4.17 Intensity of scattered light from $80 \mathrm{~nm}$ a) $\mathrm{Ag}$ and b) Al nanohemi-

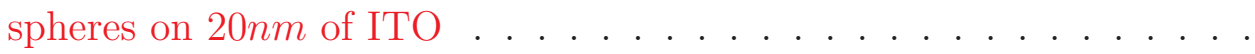


4.18 Best $J_{s c}$ for any surface coverage of $\mathrm{Al}$ nanocubes and nanospheres on increasingly thick ITO and $\mathrm{SIO}_{2}$ spacer layers. . . . . . . . . . . . . 71

$4.19|E|$ at the solar spectrum peak, 500nm, on a plane passing through the center of the particle parallel to the direction of polarization. Field intensity shown for 80nm a) Al cubes, b) Ag cubes and c) Ag hemispheres on $20 n m$ ITO on a-Si. . . . . . . . . . . . . . . . .

4.20 Generation rate (charge pairs $/ \mathrm{m}^{3} / \mathrm{s}$ ) in a-Si solar cells coated with $20 \mathrm{~nm}$ ITO and $25 \%$ surface coverages of $80 \mathrm{~nm}$ a) Al cubes, b) Ag cubes, c) Ag hemispheres and d) no MNPs. The lines show the approximate $x$-position of the MNPs. All colour scales are identical. . .

$4.2180 \mathrm{~nm} \mathrm{Al}$ cube on $20 \mathrm{~nm}$ ITO showing resonant modes as $\mathrm{Al}$ absorption peaks, mostly in the UV. The dipolar (i) and quadrupolar(ii) modes are shown at $483 \mathrm{~nm}$ and $315 \mathrm{~nm}$ respectively. . . . . . . . . . . . . . 74

4.22 Simulated I-V curve for a) Al nanocubes and b) Ag nanocubes on a $20 \mathrm{~nm}$ ITO spacer layer. Efficiencies are $7.51 \%$ and $6.56 \%$ respectively, a cell with no MNPs is $6.45 \%$ efficient. . . . . . . . . . . . . 75

5.1 Thin-film nc-Si cell cut-out profile . . . . . . . . . . . . . 78

5.2 a) L-Edit mask for defining solar cell back Al contacts, active ITO area and top $\mathrm{Al}$ contacts and b) actual processed wafer with ITO top contacts defined. . . . . . . . . . . . . . . 79

5.3 nc-Si surface with $20 \mathrm{~nm}$ ITO deposited. . . . . . . . . . . . . . . . 80

5.4 Fully processed $500 \mu m \times 500 \mu m$ array after Ag top electrode deposition. The grey box outlines the nanocube array and the larger blue box outlines the ITO active area. . . . . . . . . . . . .

5.5 SEM images showing top down view of Al nanocube arrays with pitches

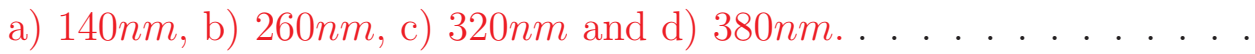


5.6 SEM image of nc-Si with $20 \mathrm{~nm}$ ITO taken at $9 \mathrm{k} \times$ magnification. . .

5.7 Zoomed out SEM image of $140 \mathrm{~nm}$ pitch array taken at $10 \mathrm{k} \times$ magnification. Nanocube array is clearly visible as faint grid structure. . . .

5.8 Reflectance simulations and measurements for a reference cell with $20 \mathrm{~nm}$ of ITO and a cell with Al nanocube array at $380 \mathrm{~nm}$ pitch. . . .

5.9 Reflectance simulations and measurements for a reference cell with $20 \mathrm{~nm}$ of ITO and a cell with Al nanocube array at $140 \mathrm{~nm}$ pitch. Also shown is the reflectance of 300nm thick Ag surface on nc-Si. . . . . .

5.10 Reflectance simulations and measurements for a reference cell with $20 \mathrm{~nm}$ of ITO and a cell with Al nanocube array at $320 \mathrm{~nm}$ pitch. . . .

$5.1180 \mathrm{~nm} \mathrm{Al}$ nanocube array with $140 \mathrm{~nm}$ pitch. Image taken at $25^{\circ}$ inclination and $60 \mathrm{k} \times$ magnification. . . . . . . . . . . . .

5.12 a) Raw EQE data for cells with varying array pitch b) Normalized EQE assuming beam is non-uniform with a Gaussian distribution $(\sigma=0.15)$

5.13 Experimental $\mathrm{I}-\mathrm{V}$ curve for a $1 \mathrm{~mm} \times 1 \mathrm{~mm}$ defined active area nc-Si solar cell with no MNPs integrated. . . . . . . . . . . . . . . . . . . . 91

B.1 L-Edit wafer mask layout . . . . . . . . . . . . . . . . . . . . 112 


\section{Nomenclature}

\section{List of Abbreviations}

- LSPR: localized surface plasmon resonance

- SPP: (propagating) surface plasmon polariton

- CVD: chemical vapour deposition

- PECVD: plasma enhanced chemical vapour deposition

- a-Si: amorphous silicon or hydrogenated amorphous silicon

- nc-Si: nanocrystalline silicon

- pc-Si: protocrystalline silicon

- $\mathrm{eV}$ : electron volt

- MNP: metal nanoparticle

- TCO: transparent conducting oxide

- ITO: indium-doped tin oxide, a common TCO

- UV: light in the ultraviolet portion of the electromagnetic spectrum

- VIS: light in the visible portion of the electromagnetic spectrum 
- EM: electromagnetic (field)

- TIR: total internal reflection

- FDTD: finite difference time domain

- AM1.5G: air mass 1.5 global, refers to the amount of solar light (direct and diffuse) reaching the ground at a solar zenith angle of $48.2^{\circ}$

- EQE: external quantum efficiency

- IQE: internal quantum efficiency

\section{List of Symbols}

- E: electric field

- H: magnetic field

- B: magnetic induction

- $\varepsilon_{0}$ : permittivity of free space

- $\varepsilon$ : relative permittivity

- $\lambda$ : wavelength

- $\omega: \omega=2 \pi f$ is the angular frequency

- $\mu_{0}$ : permeability of free space

- $\sigma$ : optical conductivity 


\section{Chapter 1}

\section{Introduction}

"We are like tenant farmers chopping down the fence around our house for fuel when we should be using Nature's inexhaustible sources of energy - sun, wind and tide.

... I'd put my money on the sun and solar energy. What a source of power! I hope we don't have to wait until oil and coal run out before we tackle that." -Thomas Edison

One hundred years ago Thomas Edison knew what we as a society are only just beginning to realize today. However, that is changing, as climatologists and scientists around the world now agree that humans are causing climate change, and a massive push is slowly being made into cleaner, less carbon-intensive forms of energy. In 2006, the US Department of Energy released a study outlining carbon intensity limits on our energy supply. They showed that by 2050 the world will need 15 TW of carbon neutral power (compared to the $13.5 \mathrm{TW}$ global consumption in 2001 and projected 27.6 TW by 2050) to limit the effects of climate change, and that only solar and wind energy have enough extractable potential energy to meet demand [2]. Of these, solar energy is the only viable source based on global land-use requirements.

Solar energy is an abundant, clean and readily available source of power, with the 
raw amount of solar energy reaching Earth's surface exceeding 9000× current global energy consumption [3] implying that $15 \mathrm{TW}$ is achievable with $10 \%$ efficient cells covering only .17\% of Earth's surface, or the land area of Venezuela. However, the actual amount of energy available for use is somewhat lower, and will depend on a region's geographic position, typical weather conditions, and land availability. Using estimates for the maximum amount of available land area for solar energy collection, as well as average weather and climate in differing regions, solar energy potential on Earth is a maximum of 49,837 petawatt hours (PWh) per year or $\sim 124 \times$ current global consumption [3].

Solar photovoltaic cells are able to capture the incoming solar energy and produce carbon free electricity; however, a major challenge facing the solar photovoltaic industry is the relatively low conversion efficiency of solar cells and correspondingly high costs. On this front, costs have indeed dropped drastically in the past few years, with crystalline silicon (c-Si) solar module costs declining from $\$ 1.37 / W$ in 2011 to $\$ 0.74 / W$ by the end of 2013 [4], a $46 \%$ drop in only 3 years. Nonetheless, there is still a need for greatly improved solar cell efficiency in order to bring down costs further and achieve parity with fossil fuel power sources in all regions of the world.

The current solar market is dominated by c-Si solar cells due to their falling costs and relatively high efficiencies $(\sim 15 \%-22 \%)$ compared to thin-film solar cell architectures. However, c-Si represents an inefficient use of materials since most of the thickness is simply mechanical support not required for efficient absorption. c-Si is mechanically cut from a bulk wafer of silicon and is constrained to $\sim 200 \mu \mathrm{m}$ or more. Since photons must be absorbed within a diffusion length of the junction in order to be collected, extraordinarily long carrier lifetimes are required. To create the necessary carrier lifetimes the silicon must be $99.999 \%$ pure, adding expensive and energy intensive steps before wafer processing even starts. Due to these thickness and 
purity requirements for c-Si, material costs are a large part of overall solar module cost. The purification process also adds significantly to the energy payback time for c-Si solar cells, accounting for almost $80 \%$ of the embodied energy [5].

Energy payback time varies significantly between technologies and manufacturers, and can be calculated as

$$
\mathrm{T}_{\mathrm{TPB}}=\frac{\text { direct }+ \text { indirect energy invested in module }}{\text { energy produced by a module }}
$$

where the energy invested involves hidden energy in the processing materials as well as the processing to purify the silicon in the case of c-Si. In 2000, energy payback times were around 2-5 years for $15 \%$ efficient c-Si solar modules [5]. Unfortunately c-Si solar cell manufacturing has not achieved significant reductions from this point and in 2014 the energy payback time still sits around 1-2 years for c-Si modules [6].

This is where a thin-film technology has a significant advantage since the energy needed for production of a thin-film material is significantly less than that used for c-Si. Reduction of material costs and energy payback times is possible if the material thickness is significantly decreased while maintaining high conversion efficiencies. These ultra-thin cells, on the order of $100 \mathrm{~nm}-1 \mu \mathrm{m}$ are dubbed "thin-film solar cells" and can be made of many different materials including cadmium telluride (CdTe), copper indium gallium selenide (CIGS) or amorphous silicon (a-Si), all of which have vastly higher absorption coefficients than conventional bulk c-Si as shown in Fig. 1.1.

These thin-film materials have significant advantages over c-Si solar cells in terms of carbon emissions from manufacturing. Thin-film cells eliminate the silicon melting, drawing and slicing used in traditional c-Si cells, reducing energy and carbon intensity significantly. This is especially important in the current solar market as almost half of all c-Si cells are produced in China. Recent studies showed that cells manufactured 
in China have double the carbon footprint of those manufactured in Europe, with European cells having a footprint of $35 g \mathrm{CO}_{2} / k W h$, assuming a useful cell lifetime of 30 years [6].

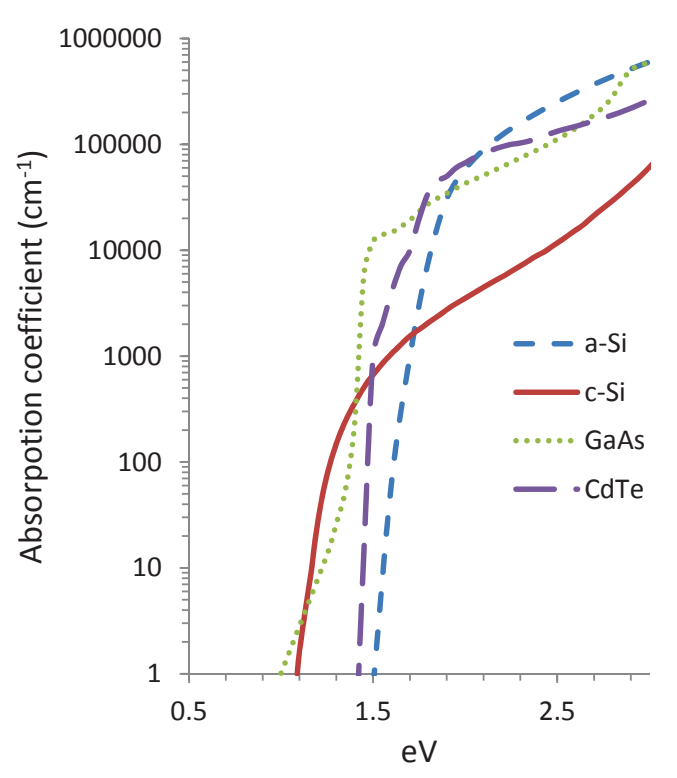

Figure 1.1: Absorption coefficients of common solar cell absorber materials in the solar spectrum

Even more daunting is the fact that most Chinese cells are exported to European and North American markets with installed energy bases having about half of the carbon intensity found in China. This means the payback period for counterbalancing carbon emissions used during manufacture effectively doubles compared to the situation where Chinese cells are installed in China. In that case, the high carbon intensity of the energy used and that of the energy saved would cancel each other out; but, if the cells are manufactured in a region with a high carbon intensity energy supply and installed in a region with a low carbon intensity energy supply, the payback period increases [6].

This means that in terms of greenhouse gas (GHG) emissions thin-film solar cells are a much better choice if the goal of solar energy is to reduce GHG emissions and 
Table 1.1: External parameters of the best laboratory c-Si and a-Si solar cells [1]

\begin{tabular}{ccccc}
\hline & $V_{o c}(\mathrm{~V})$ & $J_{s c}\left(\mathrm{~mA} / \mathrm{cm}^{2}\right)$ & Fill Factor & Efficiency $(\%)$ \\
\hline \hline c-Si & .706 & 42.2 & .828 & 24.7 \\
$\mathrm{a}-\mathrm{Si}$ & .965 & 14.3 & .672 & 9.3 \\
\hline
\end{tabular}

increase the sustainability of our electricity supply.

a-Si is perhaps the most interesting thin-film material due to silicon's high natural abundance and availability combined with the low toxicity of materials involved in the process. Though also possessing a high absorption coefficient, a-Si has very short carrier lifetime, limiting solar cells to a thickness of no more than 500 $\mathrm{nm}$ [7] for efficient carrier collection. Fortunately, a-Si can easily be formed into solar cells of the required thickness using chemical vapour deposition methods (CVD). On the other hand, a-Si solar cells with greater than $10 \%$ efficiency have yet to be consistently achievable, with the best research cell only attaining $13.4 \%$ efficiency after inclusion of nanocrystalline silicon (nc-Si) in parts of the cell. The nc-Si stabilized the cell and prevented light-induced degradation, otherwise known as the Staebler-Wronski effect. The Staebler-Wronski effect is one of the main reasons that a-Si solar cells do not enjoy a dominant market share, since exposure to light reduces efficiency significantly.

A comparison of best in class single junction c-Si and stabilized a-Si solar cells is detailed in Table 1.1. Clearly, a-Si solar cells must be significantly less expensive in order to maintain the same or lower cost per Watt of installed power. Several years ago that was indeed the case; however, today c-Si of sufficient quality has dropped drastically in price necessitating further work into increasing the efficiency of a-Si solar cells. Bringing up the market share of a-Si cells very important in order to drive reductions in the carbon intensity of solar cell manufacturing and reduce greenhouse gas emissions. 
A further impediment for commercialization of higher efficiency a-Si solar cells is the CVD process. CVD is currently a slow method for silicon deposition; however, there is much research and development currently going into developing higher throughput CVD methods for use in extremely fast roll-to-roll manufacturing of a-Si solar cells $[8,9]$. Such a manufacturing process could reduce solar cell costs dramatically and a-Si is the best candidate for this process due to its low toxicity, low cost, high abundance of raw materials and straightforward deposition process [10], provided a-Si solar cell efficiencies can be increased.

The main issue with all types of thin-film solar cells is the short optical path length through the device due to material thickness. To efficiently capture all of the light generated carriers, solar cells must have a minority carrier diffusion length which is several times longer than the material thickness (i.e the absorber should be thin); but, for photons to be efficiently absorbed, the material must be optically thick. The probability of photon absorption is well known and is sometimes referred to as Lambert's law, which states that light absorption in a material goes as $1-e^{(-\alpha d)}$ where $\alpha$ is the, fixed, frequency dependent attenuation coefficient of the absorber material and $d$ is the optical path length.

$\alpha$ is fixed for the material and therefore only $d$ can be increased. To do this light must be trapped inside the absorber layer through some method in order to increase the path length and probability of photon absorption. Traditional methods of light trapping use surface texturing of the silicon and texturing of the substrate material. This reduces reflection while refracting light into the silicon at oblique angles causing light trapping by total internal reflection (TIR) and path length enhancements of up to $4 n^{2}$ or $\sim 50 \times$ are possible. For c-Si/air interface the critical angle is around $14.5^{\circ}$ meaning light reflected from the back surface must hit the front surface at an angle of $14.5^{\circ}$ or greater in order to remain trapped inside the silicon layer. 
The escape cone is even smaller at a-Si/air interfaces (critical angle $13.5^{\circ}$ ) however, front and back surface textures formed by mechanical or chemical means would be larger than the thickness of the a-Si layer and additionally back surface texturing leads to material cracking and increased recombination due to larger surface area [11]. Thus, it is desired to find a different, more suitable, mechanism for light trapping and path length enhancement in thin-film solar cells. An advanced light trapping method which meets or surpasses the $4 n^{2}$ limit, combined with a faster CVD method could drive a breakthrough in terms of much lower $\$ / W$ att for silicon solar cells.

Recently, the concept of surface plasmon supporting nanostructures has been of great research interest as a potential method for scattering light into the absorbing layers of thin-film solar cells. Surface plasmons reside at a metal-dielectric interface and are created by the interaction of light with metal nanoparticles on the sub-wavelength scale. Surface plasmons are the collective oscillations of conduction electrons, supported at a metal/dielectric interface and excited by incident light. They have the ability to guide and trap light in small volumes and exhibit widely tunable resonance conditions, making them an ideal engineering tool for light trapping. Plasmonic metal nanoparticles on the order of $100 \mathrm{~nm}$ integrated on the top surface of solar cells also

avoid all of the issues arising from traditional surface texturing methods such as recombination and cracking. In light of recent advances in the field, the goal of this thesis is to investigate and optimize the light trapping effects of plasmonic metal nanoparticles for application to thin-film silicon solar cells.

\section{$1.1 \quad$ Thesis Objectives}

Thin-film a-Si solar cells provide many benefits over bulk c-Si solar cells. The absorption coefficient of a-Si is $\sim 100 \times$ higher than that of c-Si allowing for greater 
than $90 \%$ absorption with less than $1 \mu m$ of material, bringing about tremendous cost savings. Unfortunately, the electrical quality of a-Si is rather poor compared to c-Si, requiring the use of a much thinner active layer. This thin layer necessarily reduces photon path length through the absorber and requires the use of a light trapping method. Usually front or rear surface texturing is used to facilitate light trapping in bulk silicon solar cells; however, these features are much thicker than the thin-film active layer and are not suitable for a-Si solar cells. An alternative method to surface texturing is the use of surface plasmon supporting metal nanoparticles for advanced light trapping.

The goal of this thesis is to study and optimize the use of plasmonic metal nanoparticles for light trapping and efficiency enhancement in thin-film a-Si solar cells, as this combination has the potential to unlock large cost reductions and performance enhancements in global solar energy collection.

Chapter 3 reviews the theory and physics behind thin-film solar cells and requirements for efficient operation. The concept and theory of surface plasmons is also discussed and the groundwork is laid for the investigations of Chapter 4 into specific nanoparticle configurations which have the ability to excite surface plasmons near the solar spectrum. Surface plasmons are the collective oscillations of conduction electrons, supported at a metal/dielectric interface and excited by incident light. Coupling of light to surface plasmons is achieved through sub-wavelength structures and, once excited the surface plasmons decay radiatively, or non-radiatively, resulting in scattering or absorption of light. This decay can be tuned appropriately to enhance photocurrent generation inside the active layer.

Detailed theoretical investigations via numerical finite-difference-time-domain simulations are performed in Chapter 4 to examine the effect of light scattering and absorption by small metal nanoparticles placed on or near a high index substrate. 
The nanoparticles are optimized for low particle absorption and optimal performance as forward light scattering objects in solar cell applications in terms of shape, size, dielectric environment and nanoparticle material.

Chapter 5 outlines methods and procedures for fabrication of a thin-film solar cell. The remainder of the chapter demonstrates the experimental results before and after incorporation of $\mathrm{Al}$ nanocubes on the top surface of thin-film nanocrystalline solar cells. Nanocrystalline silicon is used due to its superior electrical properties and almost identical optical properties. Fabrication parameters are used that best match the optimal parameters from Chapter 4 and the outcomes help to validate the numerical results achieved by FDTD simulations.

Finally, Chapter 6 reviews the present work and achievements and outlines ideas for future progress in pursuit of high efficiency light trapping solutions for thin-film solar cells. 


\section{Chapter 2}

\section{Literature Review}

In the past few years, plasmonics has been demonstrated extensively in solar cell applications; however, the concept of light absorption enhancement due to the scattering properties of small metal nanoparticles (MNPs) supporting surface plasmons was only introduced with the 1998 pioneering work of Stuart et al. [12]. They deposited 108nm silver (Ag) metal-island films on the top surface of $160 \mathrm{~nm}$ thick SOI photodetectors and reported an almost 20-fold enhancement in photocurrent at $800 \mathrm{~nm}$. Only a few years later the field exploded, with many investigators applying the concept to light trapping and light absorption enhancement in thin-film solar cells and improving upon the design parameters governing the position and effect of the surface plasmon resonances.

There are four commonly used metals which are known to support surface plasmon resonances at optical frequencies, gold $(\mathrm{Au})$, copper $(\mathrm{Cu})$, silver $(\mathrm{Ag})$ and aluminium (Al) [13]. In 2003, Kelly et al. [14] studied the effect of size, shape and dielectric

environment on the surface plasmon resonances of Ag nanoparticles, especially the dipolar and quadrupolar resonant modes in spheres. They also examined theoretically several other shapes including spheroids and pyramids, showing the resonance shifts which occur when changing particle size or when placing the MNPs on a substrate. 
A similar modeling paper followed in 2005, in whichCatchpole et al. modeled MNPs as non-interacting, ideal dipoles on the surface of a silicon waveguide and showed that the model produced results very similar to earlier experiments, reinforcing the idea that surface plasmons have a dipole-like behaviour. It was then noticed that this effect had potential for light trapping in thin-film solar cells, opening up a whole new realm of possibilities.

Early work followed that of Stuart et al. [12], with several groups examining the properties of annealed Ag metal-island films. In 2003, Catchpole et al. [15] showed that surface plasmons could potentially couple light into the waveguide modes of thin-film solar cells and enhance photocurrent generation. They slowly evaporated a $17.5 \mathrm{~nm}$ Ag film to produce metal-islands. They demonstrated that a resonance condition existed at a wavelength of $500 \mathrm{~nm}$ and estimated that $80 \mathrm{~nm}$ particles would have the best scattering properties for application to solar cells. This paved the way for optimizations of the parameters described by Kelly et al. in thin-film solar cell applications.

The first solar cell to be examined in conjunction with plasmonics was the amorphous silicon (a-Si) thin-film solar cell in 2006. Derkacs et al. [16] exposed a thin $240 \mathrm{~nm}$ a-Si solar cell in the p-i-n configuration to 100 $\mathrm{nm}$ spherical gold (Au) nanoparticles in colloidal solution. The surface concentration of the Au particles in experiments was $\sim 3.7 \times 10^{8}$ particles $/ \mathrm{cm}^{2}$ resulting in an $8.3 \%$ increase in cell efficiency. Simulations showed that the MNPs increased the transmission of light into the cell by scattering light in the forward direction. Pillai et.al followed this with an investigation of $\mathrm{Ag}$ nanohemispheres formed by annealing a $12 \mathrm{~nm}$ layer of $\mathrm{Ag}$ on $1.25 \mu \mathrm{m}$ SOI test cells. This resulted in a 16-fold enhancement in photocurrent at $1050 \mathrm{~nm}$ and a $30 \%$ increase in solar spectrum integrated photocurrent for the device. It was also shown that an array of MNPs on the top surface of solar cells caused a reduction 
in sheet resistance on the surface and thus contributed to a higher fill factor as well as providing short circuit current $\left(J_{\mathrm{sc}}\right)$ enhancement [17].

These works proved the feasibility of using plasmonics for photocurrent enhancement in solar cells. Much more detailed explanations and attempts at optimization of the MNP arrays followed shortly thereafter. Au nanoparticles were examined again by Lim et al. [18] in a pioneering work which showed that spherical Au MNPs on the surface of a silicon $(\mathrm{Si})$ p-n photodiode led to increased photocurrent response at wavelengths longer than the plasmon resonance and a decrease in photocurrent response at wavelengths shorter than the plasmon resonance. This phenomenon was attributed to the phase difference in the light scattered by the plasmonic MNP and the light transmitted across the Si interface, with the phase difference being caused by the polarizability of the MNP at different wavelengths.

It was also found via experiment that the strength of the interaction between $\mathrm{Au}$ nanodisks and the underlying substrate was crucial in determining whether there was photocurrent enhancement or suppression [19]. This lead to a study by Catchpole et al. [20] who determined that the role of a thin dielectric spacer layer between the MNPs and the substrate was extremely important in determining the scattering properties of MNPs, and that the scattering cross section of MNPs could be manipulated by varying the distance of the particles from the substrate.

Beck et al. [21] demonstrates this further, annealing Ag films to get 30\% surface coverage of $\sim 70 \mathrm{~nm}$ hemispheres on $30 \mathrm{~nm} \mathrm{SiO}_{2}, \mathrm{Si}_{3} \mathrm{~N}_{4}$ and $\mathrm{TiO}_{2}$ spacer layers. They achieved a 5-fold increase in optical absorption at 1100 $\mathrm{nm}$ in a c-Si solar cell for particles on a $\mathrm{TiO}_{2}$ spacer due to the red-shifting of the plasmon resonance by the spacer.

All these works and several others $[13,16,20,22]$ have demonstrated the potential of plasmonic MNPs in enhancing path length and light absorption, particularly in 
the long wavelength region where the LSPR usually resides. Shifting the LSPR as close to the long wavelength region as possible significantly increases the photocurrent generation from photons in the poorly absorbed, near-infrared part of the spectrum [23].

These articles showed that by placing silver spheres and hemispheres directly onto a high index substrate or a thin transparent conducting oxide (TCO) layer the plasmon resonance is indeed red-shifted towards the near-IR region, increasing light trapping at those wavelengths. In fact, most of the previous works have focused solely on the long wavelength region to the detriment of the short wavelengths, where all noticed a significant drop in photocurrent and light absorption below the main (dipolar) plasmon resonance for silver (Ag) and gold (Au) MNPs [18,21, 24,25]. This reduction at shorter wavelengths was also noticed in terms of reduced EQE below the surface plasmon resonance $[18,19]$ and can be explained by the Fano effect, i.e, the destructive interference between the scattered and unscattered (transmitted) light at wavelengths shorter than the plasmon resonance wavelength [26].

A large potential increase in performance for solar cell enhancement by plasmonic MNPs should be possible if the short wavelength reduction is adequately addressed and mitigated while maintaining acceptable scattering at longer wavelengths. This was partially solved by placing particles on the rear of the cells $[27,28]$ at the expense of increased recombination sites in the active layer, causing undesirable electrical properties and problems with carrier collection. A second solution to this issue is the use of what is referred to as off-resonance enhancement. Off-resonance enhancement is named as such due to the fact that the plasmon resonance is shifted out of the solar spectrum allowing the destructive Fano effect to occur in the harmless region below $300 \mathrm{~nm}$ where no power resides in the solar spectrum. This is in contrast to on-resonance enhancement exhibited by many earlier works where the plasmon 
resonance occurs within the solar spectrum and incident light that is in the region of the resonance wavelength of the particles is strongly scattered or absorbed, depending on the size of the particles [11]. Several very recent publications [29-31] show that if the plasmon resonance can be blue-shifted far enough, the enhancement in light absorption dominates over the majority of the usable solar spectrum, rather than showing reductions at shorter wavelengths. The trade-off is reduced enhancements at longer wavelengths, but nevertheless, this method allows for an overall improvement in solar cell light absorption.

Blue-shifts are notably achieved most easily by choice of particle material. It is notoriously difficult to significantly blue-shift plasmon resonances further than $100 \mathrm{~nm}$ by altering the shape, size or dielectric environment of the nanoparticles. A blueshift is possible with decreasing particle size, however this leads to large increases in parasitic absorption (heating losses) within the nanoparticle [32]. Red-shifting plasmon resonances on the other hand is much easier, and significant red-shifts are achievable using high index substrates, or larger particles, as was shown by many earlier works. Aluminium (Al) has the highest frequency plasmon resonance of any of the optical plasmonic metals. Choosing $\mathrm{Al}$ as the metal for plasmonic nanoparticles has shown much promise as large broadband gains in EQE and light absorption have been demonstrated [29-31,33].

Villesen et al. [29] showed the benefits of blue-shifting the plasmon resonances by fabricating a p-i-n photodiode with a $40 \mathrm{~nm}$ silicon dioxide $\left(\mathrm{SiO}_{2}\right)$ spacer layer and depositing an array of $100 \mathrm{~nm} \times 100 \mathrm{~nm} \mathrm{Al}$ cylinders with $400 \mathrm{~nm}$ pitch, realizing a $2.8 \%$ gain in integrated photocurrent over the solar spectrum. An identical array of $\mathrm{Ag}$ cylinders was shown to exhibit an integrated gain of only $1.2 \%$ due to a $2.5 \%$ loss in photocurrent from $300 \mathrm{~nm}$ to $570 \mathrm{~nm}$ compared to only $0.3 \%$ loss for $\mathrm{Al}$ nanoparticles. This proof-of-concept work demonstrates the viability of using Al MNPs over Ag 
MNPs.

Further, Al nanocylinders with 100 $\mathrm{nm}$ diameter, 50nm height and 400nm (200nm) array pitch (corresponding to 3\% (13\%) surface coverage) were fabricated on thin GaAs photodiodes by Hylton et al. [30]. They demonstrated a $22 \%(6 \%)$ increase in spectrally integrated EQE and noticed gains throughout the $400-900 \mathrm{~nm}$ (500 900 nm) spectral range. This is in contrast to similar Au and Ag arrays leading to 9\% and $2 \%$ overall decreases respectively in integrated EQE compared with the reference diode.

Another advantage of $\mathrm{Al}$ nanoparticles is the very weak effect of oxidation on resonance position or scattering performance. Akimov et al. [34] discovered that in contrast to $\mathrm{Ag}$ which forms $\mathrm{Ag}_{2} \mathrm{O}$ having a large dielectric permittivity, aluminium oxide $\left(\mathrm{Al}_{2} \mathrm{O}_{3}\right.$ has a low dielectric permittivity and therefore does not increase particle absorption or affect the resonant position significantly. They showed that the enhancements produced by $\mathrm{Al}$ nanoparticles were much more stable when oxides were present compared to significant losses seen for oxidized Ag nanoparticles.

Using $\mathrm{Al}$ as the plasmonic metal is one of the most recent developments in the field of plasmonic solar cell enhancement. Both Villesen et al. and Hylton et al. mention that their works using Al MNPs are mostly proof-of-concept and very little work has been put into optimization of the relevant MNP parameters: shape, size, surface coverage and dielectric environment. The remainder of the thesis focuses on, and expands the knowledge base for the use of Al MNPs as applied to light trapping in thin-film solar cells. 


\section{Chapter 3}

\section{Theory and Concepts}

\subsection{Thin-film a-Si solar cells}

Thin-film amorphous silicon (a-Si) solar cells are similar to bulk crystalline silicon solar cells in many aspects of operation, but, differ in a few fundamental areas presenting several challenges and opportunities for solar cell designers. In terms of optical properties, the most notable difference is the absorption coefficient which is $100 \times$ greater for a-Si, as seen in Fig. 1.1. The high absorption of a-Si allows the cell to absorb more than $90 \%$ of incident sunlight with less than $1 \mu \mathrm{m}$ of material, whereas c-Si actually needs $\sim 700 \mu \mathrm{m}$ to absorb $90 \%$ of incoming light. This is a major advantage of a-Si over c-Si; however, the real challenges of a-Si lie in its electrical properties which inhibit a $1 \mu m$ thick a-Si cell, implying that advanced optical light trapping schemes are needed for a-Si solar cells, as mentioned in the introduction. A comparison of the relevant parameters for c-Si and a-Si solar cells is presented in Table 3.1. A distinct advantage for thinner cells is that $V_{\mathrm{OC}}$ increases because thinning the cell at constant short circuit current enhances the minority carrier generation rate per unit volume, and hence the steady state carrier concentration. Thermodynamically, an increased 
Table 3.1: Comparison of c-Si and a-Si [1]

\begin{tabular}{ccc}
\hline & $\mathrm{c}-\mathrm{Si}$ & $\mathrm{a}-\mathrm{Si}$ \\
\hline \hline Diffusion length $(\mu \mathrm{m})$ & $10-200$ & $0.1-2$ \\
Electron mobility $\left(\mathrm{cm}^{2} / V \cdot s\right)$ & $500-1000$ & 10 \\
Conductivity $(\mathrm{S} / \mathrm{cm})$ & $10^{-4}-10^{4}$ & $10^{-3}-10^{-2}$ \\
Doping efficiency & 1 & $10^{-3}$ \\
\hline
\end{tabular}

carrier concentration is equivalent to a reduction of the entropy production per photon, thus leading to larger voltage [35]. Best in class $V_{O C}$ for p-i-n a-Si solar cells is $\sim 0.965 \mathrm{~V}$ and $J_{S C}=14.5 \mathrm{~mA} / \mathrm{cm}^{2}$ whereas $V_{O C}=\sim 0.7 \mathrm{~V}$ and $J_{S C}=42 \mathrm{~mA} / \mathrm{cm}^{2}$ for c-Si.

Due to the lack of crystal structure in a-Si, there are many weak bonds which break easily to form defects. These act as recombination sites, significantly reducing the electron mobility compared to c-Si. In the formation of a-Si, hydrogen can be, and almost always is, incorporated into the lattice structure to passivate the dangling bonds which reduces the number of recombination sites. The resulting material is known as a-Si:H having the atomic structure shown in Fig. 3.1, which many refer to simply as a-Si.

a-Si also suffers from light-induced degradation known as the Staebler-Wronski effect [36]. Exposure to light reduced the photoconductivity and carrier transport in the amorphous silicon material. These changes were found to be totally reversible by annealing at greater than $150^{\circ} \mathrm{C}$. A lot of research was put into mitigating the StaeblerWronski effect, most notably, it was discovered that increasing the hydrogen dilution ratio during silicon deposition decreased the degradation caused by the StaeblerWronski effect. It was found that increasing the hydrogen ratio causes the structure to become more crystalline. Materials with even small amounts of crystallinity in the 
amorphous matrix see degradation levels saturate after only a hundred hours of light exposure and at a much higher performance level than non-hydrogenated a-Si [36]. Increasing crystalline fraction until a protocrystalline state completely eliminates the Staebler-Wronski degradation, however the absorption coefficient also decreases and matches more with c-Si. Thus there is a trade-off in designing efficient a-Si solar cells which have widely varying and tunable properties based on deposition parameters.



Figure 3.1: Representation of a-Si atomic structure [1]

One key difference in the operation of c-Si versus a-Si solar cells is whether the transport mechanism for photo-generated carriers is drift, or diffusion. c-Si is a diffusion based device. A thin n-type layer is formed at the top of a $300 \mu m$ p-type wafer, creating a p-n junction. The electrons in the n-type material swiftly diffuse towards the p-type, and similarly, holes in the p-type diffuse towards the n-type. This sets up a $\sim 500 \mathrm{~nm}$ region with very few free carriers (depletion region) as the diffusion process eventually exposes positive ion cores in the n-type and negative ion cores in 
the p-type materials. This sets up an electric field which then keeps the holes and electrons separated. In c-Si solar cells, most $e^{-} / h^{+}$pairs are generated in the bulk p-type material. Electrons in the p-type region are minority carriers and diffuse towards the depletion region where they are swept across the junction via drift due to the internal electric field. Minority carriers must reach the depletion region before they are recombined. The diffusion length should therefore be large and is on the order of $200 \mu \mathrm{m}$ or more in device quality c-Si.

On the other hand, a-Si solar cells rely on drift for transport of the photo-generated carriers. In order to form a solar cell, a-Si must be doped to provide an internal electric field and to form low-loss ohmic contacts to the external electrodes. Before 1975 it was believed that a-Si could not be doped. However Spear and LeComber reported that the mixing of phosphine or diborane gases with the a-Si precursor gas, silane, during deposition allowed the conductivity of a-Si to be varied by up to a factor of $10^{8}$. Unfortunately, the doping efficiency is extremely low compared to c-Si (see Table 3.1), requiring high phosphorous concentrations to provide good conductivity.

The reason for the low doping efficiency is explained by the autocompensation model. In c-Si, $\mathrm{P}$ atoms substitute for silicon atoms in the lattice and four electrons participate in bonding, while the $5^{\text {th }}$ is left free. In a-Si however, $\mathrm{P}$ atoms only bond to three silicon neighbours. This occurs because of the absence of a rigid lattice and is more natural as phosphorous normally forms only three bonds. Most phosphorous atoms are incorporated not as ideal neutral donors $\left(P_{4}^{0}\right)$ as in c-Si, but as defectcompensated donors $\left(P_{4}^{+}+S i_{3}^{-}\right)$. In other words there is a dangling bond formed as a result of incorporation of most dopant atoms. Thus doped a-Si has extremely high defect densities compared to intrinsic a-Si or c-Si and the diffusion length is correspondingly very short (less than 10nm) [1]. Thus, a-Si cannot be used in a $p$-n junction structure as all of the generated carriers would immediately be recombined 
before carrier collection occurred.

An a-Si solar cell relying on diffusion of minority carriers would fail. Instead, a mostly defect free intrinsic a-Si layer must be inserted in between the p-type and n-type layers for all of the light absorption and separation of carriers to take place. The doped layers are then made very thin (10nm p-type, 20nm n-type) and serve to set up an internal electric field across the active intrinsic a-Si layer, which is on the order of $200-500 \mathrm{~nm}$, or about the same size as the depletion region in c-Si cells. Generated $e^{-} / h^{+}$pairs are immediately affected, and separated, by the electric field and drift towards the doped layers such that electrons are collected at the n-type layer and holes at the p-type layer.

Due to the poor conductivity of a-Si a transparent conducting oxide (TCO) must be used as the front electrode in order to facilitate efficient current collection and distribution to the external load. Otherwise, if grid contacts are used, current is only collected in the region very near the grid fingers. Thus a TCO is extremely important to the operation of a-Si solar cells and this must also be taken into account when designing anti-reflection and light trapping schemes. 


\subsection{Plasmonics}

\subsubsection{Mie Theory: An Analytical Solution to Maxwell's Equations for Spherical Particles}

Gustav Mie wrote his seminal paper introducing the analytical solution for Maxwell's equations of a single absorbing sphere of arbitrary size in 1908. The goal was to explain the experimental data seen in colour shifts of colloidal gold nanoparticle solutions. Earlier work by Lord Rayleigh had produced what is now called the Rayleigh approximation for scattering by very small spheres with size parameters in the range $x \ll 1$ where the size parameter $x$ is given by

$$
x=\frac{2 \pi r}{\lambda}
$$

Mie's work was general and applied to spheres of arbitrary size. However, when the spheres reach the Rayleigh regime Mie's solutions start to break down due to several reasons including the quantum size effect [37]. The 'Mie Regime' is usually taken to be $0.1 \leq x \leq 100$, for example an $80 \mathrm{~nm}$ particle has $x \approx 1$ at $\lambda=500 \mathrm{~nm}$, which is well within the Mie Regime.

Mie's original solutions are restricted to plane wave scattering by a homogeneous, isotropic sphere in a non-absorbing embedding medium. However, it has found many applications from nanoparticles to millimeter-sized droplets in the atmosphere and is used in solid-state physics, physical chemistry, engineering, and astrophysics. We will outline briefly here the general form of a solution for scattering by a small particle which Gustav Mie fully solved for spherical particles. The full theory is detailed in Quinten's book 'Optical Properties of Nanoparticle Systems: Mie and Beyond' [37]. 


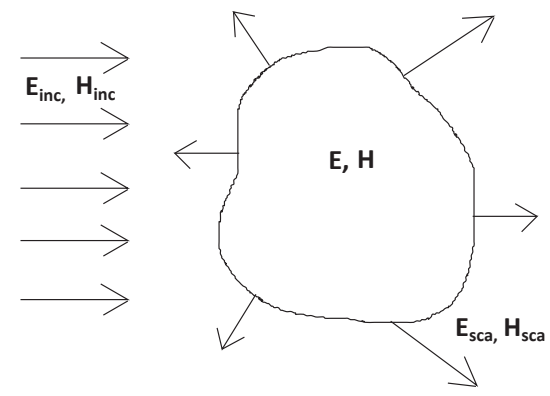

Figure 3.2: Light scattering by particle of arbitrary shape

Fig. 3.2 demonstrates schematically what occurs when an incident electromagnetic field impinges on an arbitrarily shaped particle. The field is partially refracted $(\mathbf{E}, \mathbf{H})$ and partially reflected $\left(\boldsymbol{E}_{\boldsymbol{s c a}}, \boldsymbol{H}_{\boldsymbol{s c a}}\right)$ at the surface. The reflected fields are not directed and therefore this is known as scattering. The goal is then to determine the vectorial fields $\mathbf{E}$ and $\mathbf{H}$ which satisfy Maxwell's equations and the vector wave equation inside the particle in order to use Maxwell's boundary conditions at the surface and find the resulting fields everywhere outside the particle. We assume only harmonic waves so we can separate the time dependence of the fields and write

$$
\begin{aligned}
\boldsymbol{E} & =\boldsymbol{E}(\boldsymbol{r}) \exp (-i \omega t) \\
\boldsymbol{H} & =\boldsymbol{H}(\boldsymbol{r}) \exp (-i \omega t)
\end{aligned}
$$

Thus $\mathbf{E}(\mathbf{r})$ and $\mathbf{H}(\mathbf{r})$ can be determined using Maxwell's equations:

$$
\begin{aligned}
\nabla \cdot(\varepsilon \boldsymbol{E}) & =0 \\
\nabla \cdot(\boldsymbol{B}) & =0 \\
\nabla \times \boldsymbol{E} & =i \omega \mu_{0} \boldsymbol{H} \\
\nabla \times \boldsymbol{H} & =\left(-i \varepsilon_{0} \varepsilon \omega+\sigma\right) \boldsymbol{E}
\end{aligned}
$$

where $\varepsilon$ and $\sigma$ are the relative permittivity and optical conductivity respectively. $\mathbf{E}$ 
is the electric field, $\mathbf{H}$ is the magnetic field, $\mathbf{B}$ is the magnetic induction and $\omega$ is the angular frequency. To solve this system of equations for $\mathbf{E}$ and $\mathbf{H}$ Equations 3.6 and 3.7 must be decoupled. This is done by deriving the Helmholtz equation:

$$
\nabla \times(\nabla \times \boldsymbol{Z})-k^{2} \boldsymbol{Z}=0
$$

where $\mathbf{Z}$ is either $\mathbf{E}$ or $\mathbf{H}$ and the wavenumber $k$ satisfies the dispersion relation

$$
k^{2}=\frac{\omega^{2}}{c^{2}} \tilde{n}^{2}(\omega)
$$

with $\tilde{n}(\omega)=n(\omega)+i \kappa(\omega)$.

Equation 3.8 has independent solutions for $\mathbf{E}$ and $\mathbf{H}$ due to Equations 3.6 and 3.7. We can also simplify Equation 3.4 for isotropic and homogeneous matter to $\nabla \cdot \boldsymbol{E}=0$, and combining this with Equation 3.5 means that $\nabla \cdot \boldsymbol{Z}=0$. In Cartesian coordinates, an EM field can be separated into two fields derivable from a scalar function $\phi$ satisfying the scalar wave equation. Hertz showed that one can define an EM field in terms of a single vector function $\boldsymbol{\Pi}$. If we assume that the function $\Pi_{\boldsymbol{e}}=\left(0,0, \Pi_{e}\right)^{T} \exp (-i \omega t)$ is a solution to the wave equation then

$$
\begin{aligned}
\boldsymbol{E} & =\nabla \times\left(\nabla \times \boldsymbol{\Pi}_{\boldsymbol{e}}\right) \\
\boldsymbol{H} & =\left(-i \omega \varepsilon \varepsilon_{0}+\sigma\right) \nabla \times \boldsymbol{\Pi}_{\boldsymbol{e}}
\end{aligned}
$$

where $H_{z}$ can be shown to be zero. This is a transverse magnetic (TM) mode.

Similarly, a second solution is available using the Hertz vector 
$\Pi_{\boldsymbol{h}}=\left(0,0, \Pi_{h}\right)^{T} \exp (-i \omega t)$ via

$$
\begin{aligned}
& \boldsymbol{E}=i \omega \mu_{0} \nabla \times \boldsymbol{\Pi}_{\boldsymbol{h}} \\
& \boldsymbol{H}=\nabla \times\left(\nabla \times \boldsymbol{\Pi}_{\boldsymbol{h}}\right)
\end{aligned}
$$

where $E_{z}$ can be shown as zero defining a transverse electric (TE) mode.

In the curvilinear coordinate systems (cartesian, cylindrical, spherical) if we want to now solve the boundary-value problem to determine the scattered fields outside the particle there are only complete solutions known for separable systems of cylindrical, spheroidal and spherical coordinates. Commonly vector harmonics $\boldsymbol{M}=\nabla \times(\Phi \boldsymbol{a})$ and $\boldsymbol{N}=k^{-1}(\nabla \times \boldsymbol{M})$ are used instead of Hertz vectors, where $\Phi$ satisfies the scalar wave equation and $\mathbf{a}$ is an arbitrary constant vector. Using vector harmonics reduces the problem of finding EM fields $\mathbf{E}$ and $\mathbf{H}$ that correspond to TE or TM waves to one of finding a solution $\Phi$ to the scalar wave equation, $\left(\nabla^{2}+k^{2}\right) \Phi=0$.

Now we must relate the fields outside the particle $\left(\boldsymbol{E}_{\boldsymbol{i n c}}, \boldsymbol{H}_{\boldsymbol{i n c}}, \boldsymbol{E}_{\boldsymbol{s c a}}, \boldsymbol{H}_{\boldsymbol{s c a}}\right)$ to the fields we just solved for, $\mathbf{E}$ and $\mathbf{H}$. Maxwell's boundary conditions at the particle surface are:

$$
\begin{gathered}
\left(\boldsymbol{E}_{\text {inc }}+\boldsymbol{E}_{\text {sca }}\right) \times\left.\boldsymbol{n}\right|_{\text {surface }}=\boldsymbol{E} \times\left.\boldsymbol{n}\right|_{\text {surface }} \\
\left(\boldsymbol{H}_{\text {inc }}+\boldsymbol{H}_{\text {sca }}\right) \times\left.\boldsymbol{n}\right|_{\text {surface }}=\boldsymbol{H} \times\left.\boldsymbol{n}\right|_{\text {surface }}
\end{gathered}
$$

where $\mathbf{n}$ is the normal vector to the particle surface. In general $\mathbf{n}$ is not defined consistently for the whole surface except in the case of extreme symmetry for spheres, spheroids and infinite cylinders. This is the reason analytical solutions do not exist for anything other than those three geometries, only approximations can be made.

The last piece of information we are interested in are the scattering and absorption 




Figure 3.3: Radiant flux through volume containing the entirety of the particle

cross-sections as these are experimentally measurable and a great comparison between particles of differing shapes and materials. To find the cross-sections, the rate of scattering and absorption is obtained from the energy flux balance to and from the particle. Energy changes in time over a volume enclosing the whole particle as

$$
\frac{\delta}{\delta t} \omega=\frac{\delta}{\delta t}(\boldsymbol{D} \cdot \boldsymbol{E}+\boldsymbol{B} \cdot \boldsymbol{H})=\boldsymbol{J} \cdot \boldsymbol{E}+\nabla \cdot \boldsymbol{S}
$$

where $\omega$ is the energy density and $\boldsymbol{S}=\boldsymbol{E} \times \boldsymbol{H}$ is the Poynting vector outside the particle. If we assume the stationary case $\left(\frac{\delta \omega}{\delta t}=0\right)$ then by the law of conservation of energy the loss of energy in the particle by absorption $(\boldsymbol{J} \cdot \boldsymbol{E})$ equals the radiant flux outside the particle $(\nabla \cdot S)$.

Integrating Equation 3.16 over the volume $V$ depicted in Fig. 3.3, containing the particle, we can find $W_{a b s}$, the rate of absorption in the particle as

$$
W_{a b s}=\iiint \boldsymbol{J} \cdot \boldsymbol{E} d V=-\iiint \nabla \cdot \boldsymbol{S} d V=-\frac{1}{2} R e \oiint\left(\boldsymbol{E} \times \boldsymbol{H}^{*}\right) \boldsymbol{n}_{A} d A
$$

where $\boldsymbol{n}_{\boldsymbol{A}}$ is the surface normal vector and the time-averaged Poynting vector $\boldsymbol{S}=$ $\frac{1}{2} \operatorname{Re}\left(\boldsymbol{E} \times \boldsymbol{H}^{*}\right)$ is used to coincide with experimental capabilities, as optical measuring instruments cannot detect such rapidly changing fields, only the time average. The 
EM fields outside of the particle are a superposition of the incident field and the fields of the scattered EM wave. Thus the absorption rate contains the following three terms:

$$
\begin{aligned}
W_{0} & =-\frac{1}{2} \operatorname{Re} \oiint\left(\boldsymbol{E}_{\text {inc }} \times \boldsymbol{H}_{\text {inc }}^{*}\right) \\
W_{e x t} & =-\frac{1}{2} \operatorname{Re} \oiint\left(\boldsymbol{E}_{\text {inc }} \times \boldsymbol{H}_{\text {sca }}^{*}+\boldsymbol{E}_{\text {sca }} \times \boldsymbol{H}_{\text {inc }}^{*}\right) \\
W_{\text {sca }} & =-\frac{1}{2} \operatorname{Re} \oiint\left(\boldsymbol{E}_{\text {sca }} \times \boldsymbol{H}_{\text {sca }}^{*}\right)
\end{aligned}
$$

with $W_{0}$ vanishing for particles embedded in non-absorbing media since it represents the energy absorbed inside the integration volume if the particle were absent. $W_{\text {ext }}$ and $W_{\text {sca }}$ are the sphere extinction rate and scattering rate of the sphere respectively. The extinction rate represents both scattering and absorption of light by the particle. Finally, we can discover the optical cross-sections $\sigma_{e x t}$ and $\sigma_{s c a}$ by dividing $W_{\text {ext }}$ and $W_{\text {sca }}$ by the incident intensity, $I_{0}$. In order to obtain the much quoted Mie efficiencies we divide the optical cross-section, $\sigma$, by the geometric cross-section to obtain the unitless efficiencies $Q_{\text {ext }}$ and $Q_{\text {sca }}$. $Q_{\text {abs }}$, the absorption efficiency, is determined simply by

$$
Q_{a b s}=Q_{e x t}-Q_{s c a}
$$

It wasn't until decades later in 1931 that Schopper described the behaviour of nanoparticle resonances seen in the Mie solutions as plasmons, which allowed a physical, and much more intuitive, explanation for the resonant behaviour. With the advent of numerical solvers for Maxwell's equations, such as the FDTD method, the effect of surface plasmons on arbitrary geometries is much better understood. Mie's solutions are still relevant however, in that they provide a quick reference as to where 
the resonances should lie and a mathematical explanation for the resonant behaviour. Mie's solutions remain the only fully general analytical solutions to describe the interaction of light with sub-wavelength sized particles. In the next section we will examine more closely the idea of surface plasmons and how that interpretation allows metal nanoparticles to be used for enhancement of thin-film solar cells.

\subsubsection{Surface Plasmons}

In metal nanoparticles much smaller than the wavelength of light, optical properties of the metal no longer follow that of bulk material, and are instead described best by the induced oscillations of their conduction band electrons. These are known as surface plasmon polaritons (SPPs). The term plasmon is used to refer to plasma oscillations in metals. A polariton refers to a plasma oscillation excited by incident electromagnetic waves and the term surface is used because a surface polarization is the origin of the plasma oscillation.

The occurrences of plasmons in planar metallic-dielectric interfaces and particles differ from each other, due to their different boundary conditions. Therefore, the surface plasmon polariton resonance in metal nanoparticles is also known in the literature as a localized surface plasmon resonance (LSPR) to distinguish it from the propagating SPP mode at a planar metal-dielectric interface.

Metal nanoparticles (MNPs), around 100 $\mathrm{nm}$ or less in diameter, are of great practical interest in the field of thin-film photovoltaics due to their ability to scatter incident radiation, as seen in the analytical solutions in Section 3.2.1. The condition for excitation of an LSPR is the existence of free charge carriers [37] which can

act as a polarizable medium. As light propagates through the particle, the electric field inside drives the conduction band electrons in step with the incoming EM 


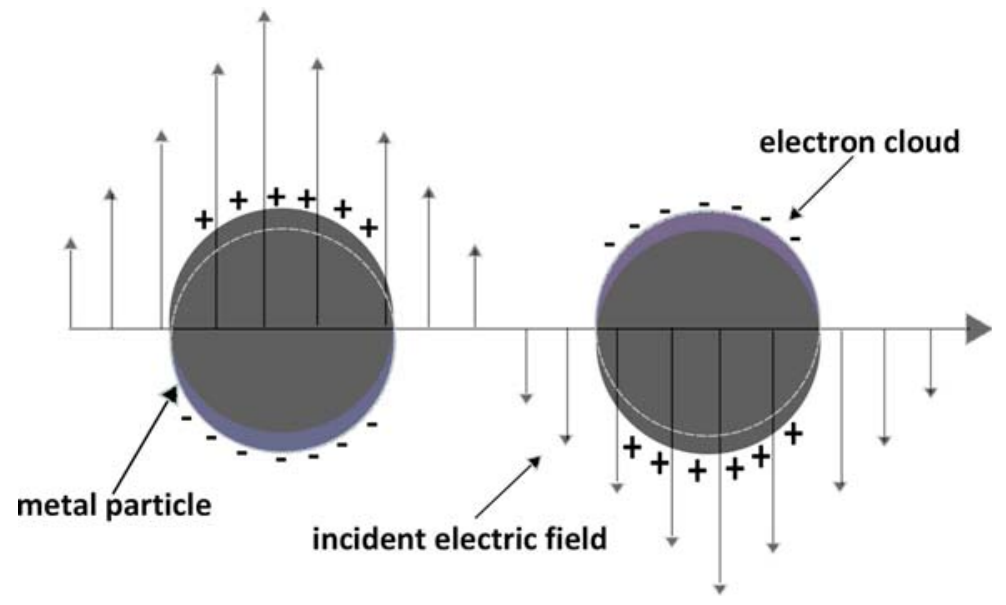

(a)

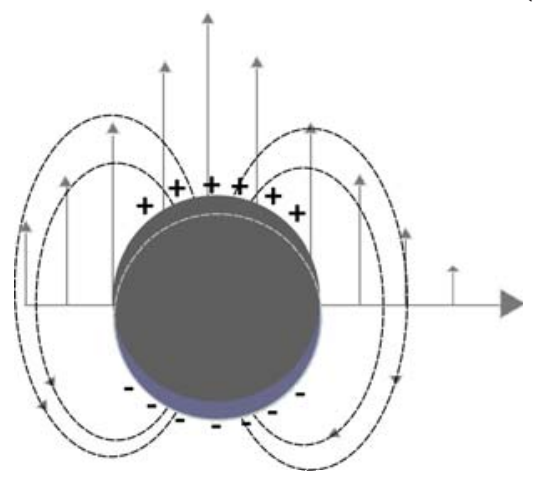

(b)

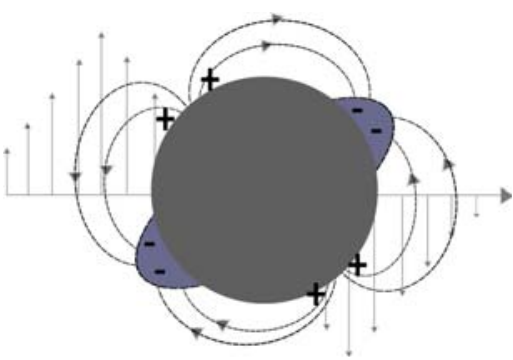

(c)

Figure 3.4: a) Interaction of a small metal nanoparticle with incident light. Also depicted is a particle exhibiting b) dipole radiation and c) quadrupole radiation for a larger particle

field. The collective movement of these conduction electrons, with respect to the fixed lattice of positive ions, leads to a build-up of polarization charges on the particle surface. The build-up of charge in conjunction with the fixed positive lattice creates a restoring force causing a plasma oscillation. These collective and coherent oscillations of electrons cause a displacement of the electrons from their nuclei, leading to the formation of various possible distributions in the surface charges. Each type of surface charge distribution is characterized by a collective oscillation mode 
(a localized surface plasmon). Differing shapes of particles will have different possible surface charge distributions leading to highly varied interactions with incoming light. Fig. 3.4a demonstrates schematically the oscillations of the conduction band electrons of a sphere in response to an impinging electric field. Here the positive ions (dark grey) remain fixed while the electron cloud (purple) oscillates back and forth with the incoming EM field. A cube for example exhibits 6 major surface charge distributions (leading to 6 main resonant modes) and an infinite number of smaller modes corresponding to charge concentrations on cube corners, edges, sides and varying combinations of the three [38], whereas spheres exhibit very uniform behaviour, only allowing for a total of three different possible charge distributions [38].

At frequencies of incoming light less than or equal to the resonance frequency of the plasma oscillation, the electrons begin to oscillate together creating a plasmon cloud [39]. Large amplitude oscillations are created at the plasmon resonance frequency. Once the surface plasmon is excited the plasmon oscillations damp (decay) radiatively via a resonant scattering process [40], or non-radiatively by electronphonon interactions [41]. The particular decay channel of the electron oscillation results in either scattering (radiative decay) or absorption in the particle (non-radiative decay) [42].

The resonant frequency depends on the strength of the restoring force, which depends on the polarizability of the surrounding dielectric medium and the particle itself, as well as particle size. For very small spherical particles the oscillations form a dipole which oscillates at the same frequency as the incoming light and radiates EM waves as depicted in Fig. 3.4b. Larger particles and other shapes form quadrupoles (Fig. 3.4c) and higher order modes as well. Thus the LSPR position depends strongly on the MNP shape, size, material and dielectric environment [14], all of which affect the polarizability of the particle. Hence, the LSPR condition can be easily tuned by 
proper adjustment of the MNP characteristic parameters.

The resonant condition can be derived for a sphere by using Maxwell's boundary conditions in Equations 3.14 and 3.15 at the surface of the sphere. First, it can be shown, using vector spherical harmonics, that the scattered wave outside of a sphere has the form

$$
\begin{gathered}
\mathbf{E}_{s c a}=-E_{0} \sum_{n=1}^{\infty} \frac{i^{n}(2 n+1)}{n(n+1)}\left[b_{n} \mathbf{M}_{o n 1}^{(3)}\left(k_{M}\right)-i a_{n} \mathbf{N}_{e n 1}^{(3)}\left(k_{M}\right)\right] \\
\mathbf{H}_{s c a}=\frac{k_{M} E_{0}}{\omega \mu_{0}} \sum_{n=1}^{\infty} \frac{i^{n}(2 n+1)}{n(n+1)}\left[a_{n} \mathbf{M}_{e n 1}^{(3)}\left(k_{M}\right)-i b_{n} \mathbf{N}_{o n 1}^{(3)}\left(k_{M}\right)\right]
\end{gathered}
$$

where $a_{n}$ and $b_{n}$ are the expansion coefficients of the TM and TE modes of the scattered wave. In order to solve for the expansion coefficients we apply Maxwell's boundary conditions at the surface of the particle using Equations 3.14 and 3.15, which, after some work gives

$$
\begin{aligned}
& a_{n}=\frac{\Psi_{n}(x) \Psi^{\prime}(m x)-m \Psi_{n}^{\prime}(x) \Psi_{n}(m x)}{\xi_{n}(x) \Psi_{n}^{\prime}(m x)-\xi_{n}^{\prime}(x) \Psi_{n}(m x)} \\
& b_{n}=\frac{m \Psi_{n}(x) \Psi^{\prime}(m x)-\Psi_{n}^{\prime}(x) \Psi_{n}(m x)}{m \xi_{n}(x) \Psi_{n}^{\prime}(m x)-\xi_{n}^{\prime}(x) \Psi_{n}(m x)}
\end{aligned}
$$

where $\Psi_{n}(x)=x j_{n}(x)$ and $\xi_{n}(x)=x h_{n}(x)$ are Riccati-Bessel and Riccati-Hankel functions and $m=n_{p} / n_{M}, x=k_{M} R$ and $k_{M}=\left(2 \pi n_{M}\right) / \lambda$, where $n_{p}$ and $n_{M}$ are the refractive indices of the nanoparticle and the embedding medium respectively. Now we note that the Riccati functions are oscillating functions and thus the denominator of the scattering coefficients can be very small if the following condition is satisfied for the $n^{\text {th }}$ TM mode

$$
m \frac{\xi_{n}^{\prime}(x)}{\xi_{n}^{\prime}(x)}=\frac{\Psi_{n}^{\prime}(m x)}{\Psi_{n}(m x)}
$$


and the condition

$$
\frac{1}{m} \frac{\xi_{n}^{\prime}(x)}{\xi_{n}^{\prime}(x)}=\frac{\Psi_{n}^{\prime}(m x)}{\Psi_{n}(m x)}
$$

is satisfied for the $n^{\text {th }}$ TE mode.

The above equations can be fulfilled in the case of free electrons, which give rise to the aforementioned LSPRs. To examine what happens, we assume the particles are very small such that Equations 3.26 and 3.27 can be approximated by the first term in their series expansion. Equation 3.26 becomes

$$
\varepsilon=-\frac{n+1}{n} \varepsilon_{M}
$$

and Equation 3.27 becomes

$$
\varepsilon=\varepsilon_{M}
$$

where $\varepsilon$ and $\varepsilon_{M}$ are the relative permittivities of the particle and embedding material respectively. The first order (dipole) LSPR for a spherical MNP in a dielectric medium occurs for $n=1$ when the following well known relationship is satisfied

$$
\varepsilon_{\text {particle }} \approx-2 \varepsilon_{\mathrm{M}}
$$

where $\varepsilon_{M}$ is the dielectric constant of the embedding medium. In this quasistatic approximation the spatial variation of the electromagnetic (EM) field is constant over the particle, producing a dipole oscillation and problem reduces from the electrodynamic one to an electrostatic one. That is, the incident electric field induces a dipole moment in the particle:

$$
\mathbf{p}(t)=\varepsilon_{0} \varepsilon_{M} \alpha \mathbf{E}(t)
$$


where $\alpha$ is the polarizability of the particle. The polarizability depends on the dielectric constant and the particle size, as mentioned earlier. For the simple case here, the polarizability of a sphere with volume $V$ is given by the Clausius-Mossotti expression

$$
\alpha=3 V \frac{\varepsilon-\varepsilon_{M}}{\varepsilon+2 \varepsilon_{M}}
$$

where $\varepsilon$ and $\varepsilon_{\mathrm{M}}$ are the complex dielectric functions of the metal particle and dielectric medium respectively. The scattering and absorption cross-sections are functions of particle polarizability, for a sphere:

$$
\begin{aligned}
\sigma_{s c a}(\omega) & =\frac{8 \pi^{2} \omega^{4}}{3 c^{4}}|\alpha(\omega)|^{2} \\
\sigma_{a b s}(\omega) & =\frac{2 \pi^{2} \omega}{c} \operatorname{Im}\{\alpha(\omega)\}
\end{aligned}
$$

Clearly the polarizability diverges when Equation 3.30 is satisfied, and this occurs at some particular wavelength since $\varepsilon$ and $\varepsilon_{M}$ are frequency dependent. This causes a discontinuity in $\sigma_{s c a}$ and $\sigma_{a b s}$ and we again arrive at the resonance condition for the dipole LSPR.

It is important to note that the scattering cross-section scales with the square of the particle volume (size) and the absorption scales linearly with increasing particle size. These findings were validated by Grigorchuk et al. [41] who found theoretically that the radiative decay channel increased quadratically with increasing nanosphere radius. This intuitively means that larger particles should exhibit scattering as the dominant extinction mechanism while small particles will absorb strongly. In reality, the ability of the MNPs to enhance light absorption in thin-film solar cells will depend on many things, including the size of the particle, dielectric environment and the frequency at which the LSPR occurs. Grigorchuk et al. also discovered that increasing 
the refractive index of the medium decreased the radiative damping of the particle, meaning particles in a high index medium will absorb more. The resonance position also plays a critical role as will be explored in depth in Section 4.4.

In a solar cell application, one of the most important factors is the amount of scattering (radiative decay) versus the amount of absorption (non-radiative decay) by the MNPs. The ratio of radiative decay to non-radiative decay is known as the branching ratio and is strongly dependent on particle diameter, aspect ratio and material. Ideally for solar cell applications the branching ratio should be very large, implying that most of the extinction is in the form of scattering due to radiative decay of the plasma oscillation.

A commonly used metric is the normalized scattering cross-section (or Mie scattering efficiency), $Q_{\text {scat }}$, of an arbitrary MNP. This is a measure of the amount of incoming radiation which is scattered by the MNP at any wavelength, including the LSPR, and is defined as

$$
Q_{\text {scat }}=\frac{\text { power of scattered light }(W)}{\text { incident intensity }\left(\frac{W}{m^{2}}\right) \times \text { particle cross-section }\left(m^{2}\right)},
$$

where $Q_{\text {scat }}$ is unit-less and does not depend on particle size. The normalized absorption cross-section (or Mie absorption efficiency), $Q_{\text {abs }}$, is calculated similarly

$$
Q_{\mathrm{abs}}=\frac{\text { power of absorbed } \operatorname{light}(W)}{\text { incident intensity }\left(\frac{W}{m^{2}}\right) \times \text { particle cross-section }\left(m^{2}\right)},
$$

The normalized absorption coefficient, $Q_{\text {abs }}$, determines how well a MNP absorbs the incoming light. Similarly, the normalized scatting cross-section, $Q_{\text {scat }}$, determines how well a MNP scatters incoming light. If $Q_{\text {scat }}$ is larger than one, this indicates that the plasmonic MNPs are able to affect incoming light over areas much larger than the physical particle itself. If $Q_{\mathrm{abs}}$ is less than one then the particle is only absorbing 
light over an equivalent area smaller than the physical particle cross-section. Near the dipolar plasmon resonance the particles absorb and scatter strongly [43] enabling detection of the resonance condition. The addition of the absorption and scattering efficiencies is known as the extinction efficiency, $Q_{\text {ext }}$.

Using the freely available software MiePlot ${ }^{\complement}$, which solves the analytical Mie equations to find $Q_{\text {ext }}$ for spheres of arbitrary material, the plasmon resonances for $\mathrm{Al}$ and Ag spheres in a vacuum were found and compared to their respective dielectric functions obtained from the SOPRA database [44]. In Fig. 3.5 the dashed line $\varepsilon_{1}=-2$ corresponds to the resonance condition described in Equation 3.30. The embedding medium is a vacuum so $\varepsilon_{M}=1$. The resonance condition should then occur when the real part of the dielectric function $\varepsilon_{1}=-2$. This is clear for the $8 \mathrm{~nm}$ spheres as they are not large enough to support any multipole resonances and the assumptions made in deriving the resonance condition (i.e particle size is negligible) hold. On the other hand, large spheres exhibit much more red-shifted resonance positions and higher order modes. From Equation 3.28 we expect (for $n=1$ ) a resonance around $155 \mathrm{~nm}$ in $80 \mathrm{~nm} \mathrm{Al}$ spheres. However, the $80 \mathrm{~nm} \mathrm{Al}$ spheres exhibit a dipole resonance around $250 \mathrm{~nm}$ instead. This red-shift of the resonant modes is due to retardation effects caused by the size dependence of the resonant conditions in Equations 3.26 and 3.27, as the simplification to Equation 3.28 no longer applies for large particles (i.e. the particle size is no longer negligible).

Retardation effects apply for large particles greater than 30nm in diameter [45] and can be explained in a basic manner as follows: assume a dipolar mode in a large spherical particle as in Fig. 3.4a, the distance between the positive and negative charges is approximately equal to the nanoparticle diameter (now non-negligible). Thus a change on one side of the particle is felt by the other side (having the opposite 




(a)

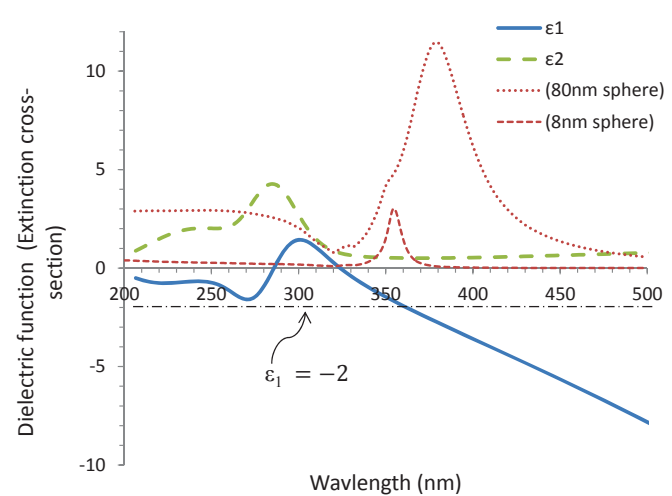

(b)

Figure 3.5: Dielectric function and extinction cross-section of a) $\mathrm{Al}$ and b) $\mathrm{Ag}$ nanospheres

charge) with a phase retardation of $2 \pi \cdot 2 R / \lambda_{M}$, where $\lambda_{M}=\lambda / \sqrt{\varepsilon_{\mathrm{M}}}$ is the effective wavelength in the embedding medium. The dipole oscillation must decrease in frequency to take into account this retardation, or equivalently, the dipole mode redshifts as particle size increases [45]. Stated slightly more eloquently by Maier: 'the bigger is the particle, the farther apart are the charges in the oscillating dipole and the smaller is the restoring force. Therefore, the resonance condition shifts to lower energies' [46]. Fig. 3.5 clearly shows the quasistatic resonance condition holding for small $\mathrm{Al}$ and $\mathrm{Ag}$ nanospheres and failing to predict the correct resonance position for the larger nanospheres. In the case of different dielectric environments, particle shapes or particle-particle interactions, which are needed to model the effects important for solar cell applications, an FDTD or FEM method is necessary to correctly account for retardation and many other effects which are not well modelled analytically.

The finite-difference time-domain method (FDTD) simulates the propagation of light through any medium in 2D or 3D. It uses Maxwell's time-domain curl equations leading to the field components $\left(E_{x}, E_{y}, E_{z}, H_{x}, H_{y}, H_{z}\right)$. The derivatives in the curl equations are found using a finite-difference approximation on a mesh grid. FDTD 
computes the field components at every grid point for every time step. It easily handles complex geometries but computation time increases exponentially with shrinking mesh sizes.

The finite-element method (FEM) obtains the approximate solution to partial differential equations (here, the wave equation) over a defined area with known boundary conditions. It uses the calculus of variations to minimize an error function and produce a stable solution. Any domain is discretized into many smaller triangular elements and the solution is determined in terms of some primary variables at each node (vertice). An approximation to the solution of the governing differential equation is found for each element. Many simple element equations over many small subdomains, named finite elements, are then connected to approximate a more complex equation over a larger domain.

In this thesis we use FDTD methods since the power absorbed and scattered by the particles and substrates is easily determined using this method. The large scattering cross-sections of MNPs indicates that they can act as excellent scattering sites in order to increase the path length of light inside the absorber layer of a thin-film silicon solar cell. 


\section{Chapter 4}

\section{Theoretical Design of Plasmonic Solar Cell}

\subsection{Simulation Methodology}

Using a 3D finite-difference time-domain (FDTD) simulator from Lumerical ${ }^{\circledR}$ we first study the optical scattering for $\mathrm{Ag}$ and $\mathrm{Al}$ nanocubes on an infinite a-Si substrate, as illustrated in Fig. 4.1. An Ag nanosphere in identical configuration is also simulated for reference. We examine the situation when a MNP sits on an a-Si substrate, sandwiching a thin $\mathrm{SiO}_{2}$ film, where $n_{\text {film }}=1.5$ and $n_{\text {subs(a-Si) }}=4.23$. Initially, the film and substrate material models used are simple, real refractive indices such that the dispersion effects of the material itself are neglected. Later, full material models taking into account the wavelength dependence of the refractive index (RI) and the material absorption are applied. Numerical simulations were checked for convergence by halving the mesh size until the results showed negligible change, decreasing the mesh further resulted in divergence again from numerical errors due to computer limitations on very small differences represented by a floating point number. The required mesh size for accurate results was $1.5 \mathrm{~nm}$ over the entire region containing 
the MNPs and substrate, resulting in high computational expense for simulations.

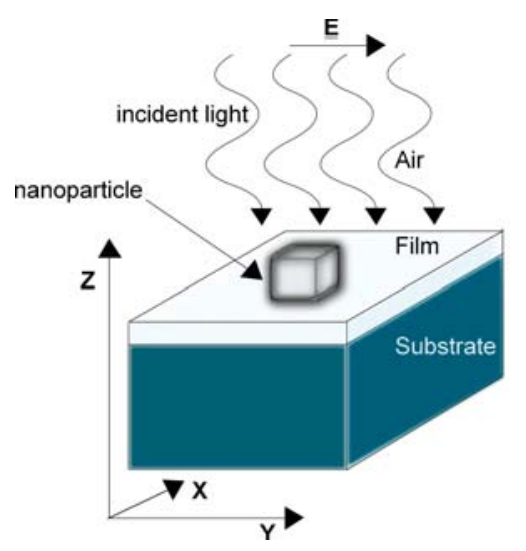

Figure 4.1: Unit cell geometry under investigation with a MNP (nanocube shown here) used as a scatterer on a thin film in contact with a semi-infinite substrate.

First the properties of individual MNPs are considered, spaced such that they are non-interacting and have no interference or grating effects, which has been shown to accurately represent a random array of MNPs [21]. Simulations were conducted to determine the scattering and absorption by the MNPs using Lumerical ${ }^{\circledR}$. A Total Field Scattered Field (TFSF) is often used to determine scattering and absorption by small particles. In Lumerical ${ }^{\circledR}$, a TFSF allows the separation of the physical field into a total field region (i.e., sum of incident and scattered waves) and a scattered field region (i.e., includes only the scattered field outside the total field region). A TFSF was placed around the MNP with illumination occurring from the upper z-plane, with perfectly matching layers (PML) for all boundary conditions to eliminate reflections from the edges of the computation window. The TFSF subtracts the incident field when it hits the source boundary so that the absorption and scattering of the incident field due to the MNPs can be obtained.

Finally a realizable a-Si solar cell geometry was simulated and full material models are used for a-Si and $\mathrm{SiO}_{2}$ in a thin-film cell structure, using Lumerical's proprietary multi-coefficient materials model for metals and $\mathrm{SiO}_{2}$. MCMs rely on a more extensive 
set of basis functions to better fit dispersion profiles that are not easily described by Drude, Debye, and Lorentz materials [47]. Material models for ITO and a-Si were taken from the SOPRA database [44] and all models were chosen to provide accurate results over the $300-900 \mathrm{~nm}$ spectrum. Following the parameters used by several other publications $[16,33,48]$ we chose a $240 \mathrm{~nm}$ absorbing a-Si layer and an $80 \mathrm{~nm} \mathrm{Al}$ back contact, with varying thickness of dielectric spacer layer on top. The Al contact serves as a back reflector in the optical simulations as $80 \mathrm{~nm}$ is thick enough to reflect back all but a negligible amount of light.

In order to find out the power absorbed in the a-Si layer solar simulations were performed using the solar_generation analysis object in Lumerical. This analysis group calculates the absorption per unit volume in the silicon. This can be accomplished by finding the divergence of the Poynting vector as $P_{a b s}=-0.5 \operatorname{Re}(\nabla \cdot P)$, but this is numerically unstable. Fortunately this is equivalent to $P_{a b s}=-0.5|E|^{2} \operatorname{Im}(\varepsilon)$, implying that to find the absorption the solver only needs electric field intensity and $\operatorname{Im}(\varepsilon)$ which are easily measurable in an FDTD simulation. The number of photons absorbed per unit volume is calculated as

$$
g=\frac{P_{a b s}}{\hbar \omega}=\frac{-0.5|E|^{2} \operatorname{Im}(\varepsilon)}{\hbar}
$$

where the total generation rate is then the integration of $g$ over the simulation spectrum $(300-900 n m)$.

Finally, the short circuit current density can be calculated as $J_{s c}=e g(A / m)$ (where $e$ is the charge on an electron) assuming that all absorbed photons generate $e^{-} / h^{+}$pairs and that all generated pairs contribute to the photocurrent. Note that this is not realistic in terms of absolute device performance, but works well as a metric to compare different devices and structures. Accurate $J_{s c}$ is obtained through 
the use of an electrical solver, Lumerical DEVICE, which utilizes the finite-element method to solve the electrical transport equations based on the spatial generation rate calculated in the FDTD simulations as described above, taking into account Auger and SRH recombination as well as surface recombination effects. DEVICE also allows measurement of overall solar cell conversion efficiency described by

$$
\eta=\frac{\mathrm{FF} \times V_{o c} \times J_{s c}}{S_{A M 1.5 G}}
$$

where FF is the fill factor, $V_{o c}$ is the open-circuit voltage and $S_{A M 1.5 G}$ is the incident power from the AM1.5G solar spectrum.

Due to computer, and even compute cluster (WestGrid HPC), memory limitations, the realizable solar cell geometries are simulated in 2D, as 3D simulations with a $1 \mathrm{~nm}$ mesh required greater than $200 G B$ of accessible memory. To alleviate some of the computational burden as well, only a single period of the structure was simulated. Periodic boundary conditions were applied in the $x$ and $z$ directions with a PML used in the $y$ direction, as $y$ was the direction of illumination. The accuracy of simulating only one period was checked by expanding the FDTD domain to cover two periods, and two MNPs, with equivalent results.

Throughout this chapter 150nm MNPs are first used when calculating and comparing properties of the MNPs themselves, whereas more optimized 80nm MNPs are later used when determining their effect on light absorption and power in a real solar cell. The larger size allows individual MNP properties to be more easily analysed and comparisons can be made providing all of the particles are of the same size. The smaller $80 \mathrm{~nm}$ MNPs retain the same general trends as their larger counterparts in terms of scattering and absorption cross-sections, but provide much larger enhancements in light absorption in a real solar cell structure allowing much easier and more 
significant comparisons. Section 4.5 will show that the $80 \mathrm{~nm}$ MNPs are the optimal size for light trapping in thin-film a-Si solar cells.

\subsection{Effect of MNP geometry on plasmonic en- hancement}

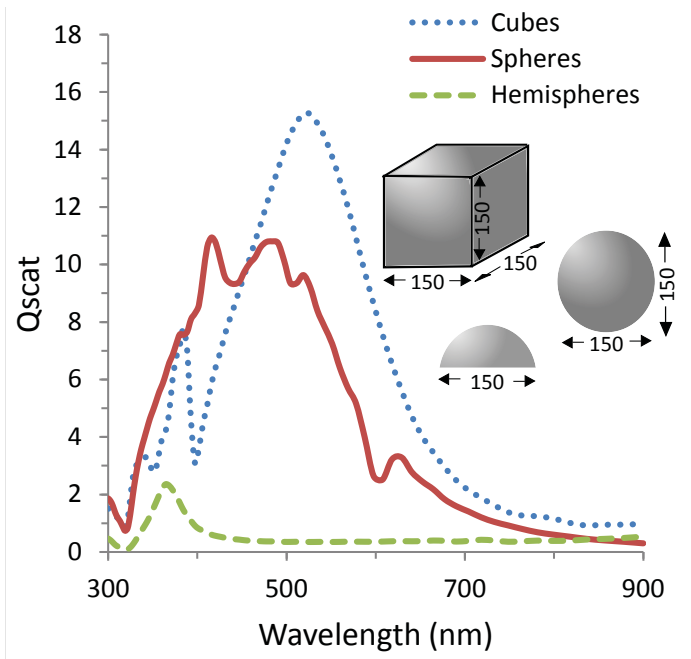

Figure 4.2: Normalized scattering cross sections of $150 \mathrm{~nm}$ Ag cubes, spheres and hemispheres sitting directly on an a-Si substrate. Inset shows MNP dimensions in $n m$.

Many shapes of MNPs have been examined in the past, including spheres, hemispheres, disks and cylinders; however, the cubic nanoparticle has received very little attention to date. We demonstrate in Fig. 4.2 that nanocubes have a scattering crosssection which is much higher than spheres or hemispheres over a broad wavelength range, therefore providing additional enhancements in light trapping and absorption over those traditional shapes. We note here that the usual thin film deposition and annealing procedure to create nanospherical particles normally produces hemispheres which can be seen to have significantly lower scattering performance than nanocubes 
or perfect nanospheres.

Cubes do have several similarities to hemispheres and disks, in that a large portion of their surface area is located in close proximity to the substrate. In the situation where these MNPs are directly on the substrate, the optical near-field couples strongly to the substrate (caused by a large area of surface contact) and alters the angular scattering distribution resulting in high backscattering of incoming radiation. Hagglund et al. [19] showed that elevating Au nanodisks off the silicon substrate reduced the near-field coupling and the backscattering.

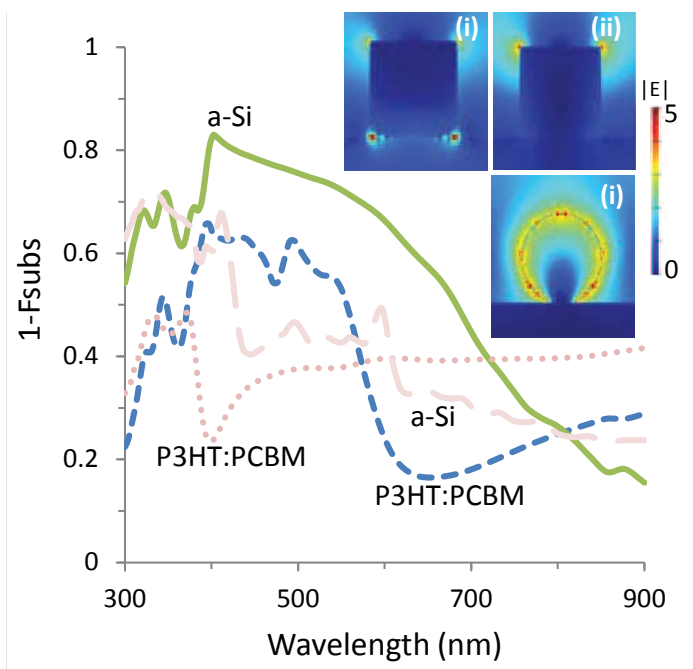

Figure 4.3: Fraction of light scattered backwards $\left(1-F_{\text {subs }}\right)$ for $150 \mathrm{~nm}$ Ag cubes sitting directly on $n_{\text {subs(P3HT:PCBM) }}=1.8$ (dashes) and $n_{\text {subs(a-Si) }}=4.23$ (solid). Also shown, 150nm Ag spheres on P3HT:PCBM (dots) and a-Si (long dashes). Inset shows electric field distribution of the dipolar (i) and quadrupolar (ii) modes of a nanocube and the dipolar (i) mode of a nanosphere directly on a-Si.

The main argument against using nanocubes in an application where the substrate has a high RI is their tendency to backscatter much of the incident radiation. We define the fraction of light forward scattered into the substrate as $F_{\text {subs }}$ and the fraction backscattered is then $1-F_{\text {subs }}$. From Fig. 4.3 we can deduce that the primary effect of transitioning from a lower index substrate $\left(n_{\text {subs( } \mathrm{P} 3 \mathrm{HT}: \mathrm{PCBM})}=1.8\right.$, a common 
absorber layer in organic solar cells), to a higher index substrate $\left(n_{\text {subs(a-Si) }}=4.23\right)$, is to increase the backscattering profile of the nanocubes. The reason for this is the location of the scattering modes in nanocubes as opposed to nanospheres. Zhang et al. [38] showed that the quadrupolar mode is predominantly on the top surface of the nanocube, while the dipolar mode is concentrated on the bottom surface (closest to substrate). Thus the dipolar, and primary scattering mode [32], is much more directly affected by coupling to the higher index substrate than it is on a nanosphere which has more symmetrically distributed modes. This is easily seen in the inset in Fig. 4.3 which shows the distribution of the dipolar $(i)$ and quadrupolar $(i i)$ modes in a nanocube and the dipolar $(i)$ mode in a nanosphere. The dipolar mode in the nanocube obviously has a large contact area with the substrate which alters its angular scattering profile and leads to increased backscattering in the presence of a high index substrate. For nanospheres, the modes are symmetrically distributed around the sphere producing much less coupling to the substrate.

In the case of nanospheres, Fig. 4.3 shows the transition from the P3HT:PCBM substrate to a-Si induces a much smaller increase in backscatter than the same transition for nanocubes over the relevant solar wavelengths for a-Si, 300 $\mathrm{nm}$ to $730 \mathrm{~nm}$. The resonant dipolar mode in nanospheres has low contact with the substrate which leads us to the same conclusion as Hagglund et al. [19], that a major factor causing backscattering by the MNPs is near-field coupling to a high index substrate. It is evident that in a low RI environment the nanocube can be a superior particle shape for broadband plasmonic enhancement compared to nanospheres or nanohemispheres. 


\subsection{Effect of a dielectric spacer layer on MNP per- formance}

Strong near-field coupling not only produces an increase in optical backscattering, but also brings about undesirable electrical properties. A thin-film silicon solar cell in pi-n configuration typically has $10-20 \mathrm{~nm}$ p-doped and n-doped regions. The thinner p-type window layer is normally at the top of the cell and extends approximately as far as the plasmonic near-field. Unfortunately, any charge carriers created by photons absorbed in the doped regions will be recombined before they can be collected [49]. Thus any near-field coupling of power to the substrate is immediately lost to recombination. Pure optical simulations will not account for this. Hence, it is important to realize that the near-field coupling should be minimized and is undesirable in $\mathrm{p}-\mathrm{i}-\mathrm{n}$ thin-film solar cells. As discussed in section 4.2, elevating the MNPs off the high index substrate could effectively reduce the near-field coupling and backscattering.

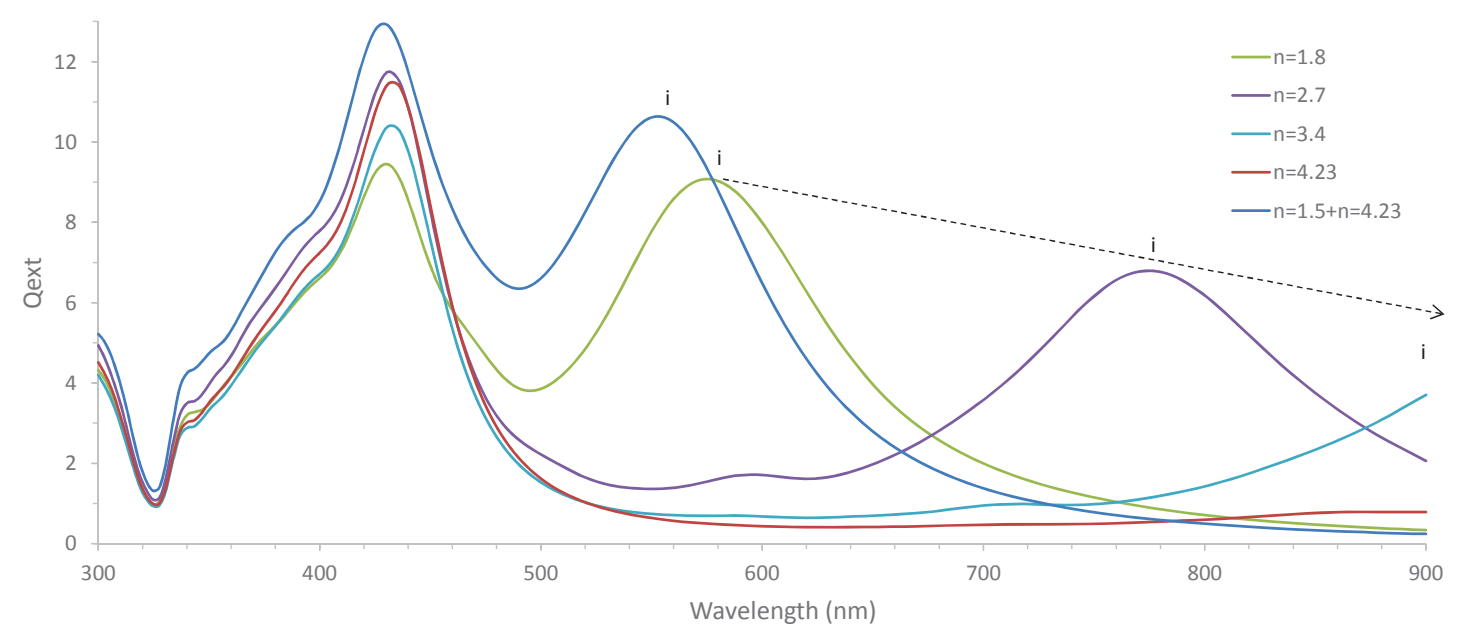

Figure 4.4: Extinction efficiency in 80 $\mathrm{nm}$ Ag nanocubes on substrates with increasing refractive indices $n_{\text {subs }}=1.8$ to $n_{\text {subs }}=4.23$. The dark blue curve reflects changes when a $25 \mathrm{~nm} \mathrm{SiO}_{2}$ spacer layer is inserted between the $\mathrm{Ag}$ nanocubes and an a-Si substrate. The resonant dipolar (i) modes are shown for each curve. 
Additionally, as seen in Fig. 4.3, an environment with a low RI is desirable for improved performance of plasmonic MNPs, especially for nanocubes. However, as a-Si is a widely used absorber for thin-film solar cells, it is desired to find a way to reduce the effective RI seen by the MNPs. This can be achieved by placing the MNPs on a thin dielectric spacer layer to minimize the effect of near-field coupling and subsequent backscatter. A spacer layer has previously been indicated as an important factor in determining the scattering properties of MNPs $[19,28,50]$, and the presence of a thin oxide layer can enhance considerably the forward scattering due to particles with a large contact area to the substrate [51]. Given an a-Si substrate, including a thin dielectric spacer layer under the MNPs can reduce the effective RI and bring large increases in the light absorption enhancement due to plasmonic nanocubes.

In choosing a dielectric, we would like a low RI material so as to reduce the effective RI seen by the MNPs. Ideally, the material used has the lowest refractive index available in a solar cell compatible material. On a practical a-Si solar cell however, it is desired to use a spacer with good electrical conductivity as well as a low RI to facilitate efficient carrier collection, as a-Si has poor lateral conductivity due to its short $(\sim 100 \mathrm{~nm})$ minority carrier diffusion length and high sheet resistance [16]. $\mathrm{SiO}_{2}$ and indium-doped tin oxide (ITO) are both possibilities, with ITO having a slightly higher RI $(n=1.9)$ than $\mathrm{SiO}_{2}(n=1.5)$, but with excellent electrical conductivity. However, in order to accentuate the effects of the low RI spacer layer, we will use $\mathrm{SiO}_{2}$ as the spacer layer since the trends observed in the optical performance of $\mathrm{SiO}_{2}$ should remain largely unchanged with ITO.

An additional issue for all MNPs, regardless of shape, is the reduction in solar cell performance due to parasitic absorption in the MNPs. Near the LSPRs, there is strong parasitic absorption of incoming light within the MNPs and greater than $40 \%$ 
of incoming power can be dissipated in the MNPs near the LSPRs [52]. Hence, blueshifting the LSPRs out of the peak of the solar spectrum and into the UV region will greatly reduce the parasitic absorption throughout the solar spectrum range relevant to a-Si.

The dielectric spacer layer along with the underlying substrate plays a critical role in red or blue-shifting the LSPRs. Due to the effects of depolarization, particles in close proximity to a higher index substrate exhibit plasmon resonances which are significantly red-shifted, especially for the lowest order modes [50]. Fig. 4.4 shows that the dipolar mode $(i)$ red-shifts more than 500 $\mathrm{nm}$ when going from a P3HT:PCBM substrate $\left(n_{\text {subs }}=1.8\right)$ to a substrate that models a-Si $\left(n_{\text {subs }}=4.23\right)$. Similarly, the quadrupolar mode $(\mathrm{ii})$ red-shifts $57 \mathrm{~nm}$. The higher order modes demonstrate little movement as they have virtually no contact with the substrate. Adding a thin $25 \mathrm{~nm}$ spacer layer of $\mathrm{SiO}_{2}$ on top of the $n_{\text {subs }}=4.23$ substrate shows a significant blue-shift of the dipolar resonance back to $550 \mathrm{~nm}$ due to the much lower surrounding refractive index as seen in Fig. 4.4. This means the resonance is widely tunable using a simple spacer layer.

The blue-shift that occurs when a thin dielectric spacer is added can be explained by the effects of dielectric polarization at the interface between the MNPs and the dielectric. This polarization is a result of the charge accumulation in the MNPs when surface plasmons are excited. The charge accumulation creates an electric field which subsequently polarizes the surrounding medium. The strength of this polarization depends solely on the dielectric permittivity, $\epsilon_{m}$, of the medium. The larger $\epsilon_{m}$, the larger the polarization strength. The polarization charge partially offsets the charge accumulation in the MNP thus reducing the restoring force created. This system behaves similar to a linear oscillator in which it is established that a reduction in 


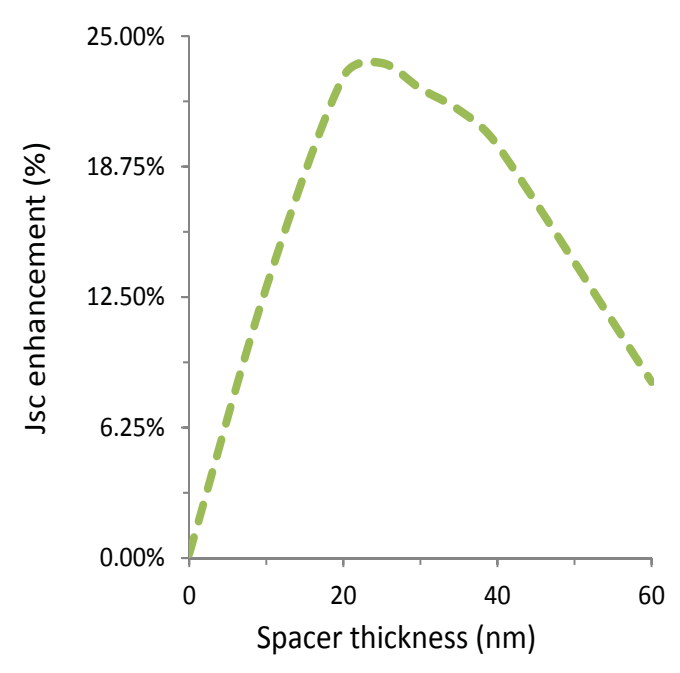

Figure 4.5: Optimization of $\mathrm{SiO}_{2}$ spacer layer thickness under a $25 \%$ surface coverage of uniformly distributed $80 \mathrm{~nm} \mathrm{Ag}$ nanocubes in terms of $J_{s c}$ enhancement over an identical cell without MNPs, assuming that each photon absorbed creates an $e^{-} / h^{+}$pair contributing to the photocurrent.

restoring force leads to a smaller resonant frequency (i.e at a longer wavelength) [43]. Therefore the plasmon resonances will be red-shifted compared to a vacuum by the presence of any medium, and will be greatly red-shifted by the presence of a high dielectric function material like a-Si. As we add a dielectric spacer film with a smaller $\epsilon_{m}$ than that of a-Si, the LSPRs should blue-shift.

Increasing the thickness of the spacer layer increases the amount of blue-shift [50] and affects the phase of the light being transmitted into the a-Si layer. As shown by Tsai et al. [53] a smaller phase difference between the transmitted light and that scattered by the MNPs generates larger enhancements in light absorption. Thus there exists an optimal spacer layer thickness. Fig. 4.5 shows the percent enhancement in short circuit current $\left(J_{s c}\right)$ in a $240 \mathrm{~nm}$ thick a-Si solar cell as a function of spacer thickness for a fixed cell design. The enhancement is compared to a cell with the appropriate spacer thickness and here we see that a $25 \mathrm{~nm}$ spacer provides the greatest enhancement in $J_{s c}$ of $\sim 24 \%$. We assume that cell efficiency directly follows changes 
in $J_{s c}$ as the MNPs on the top surface do not contribute significantly to recombination and thus do not affect the open circuit voltage $\left(V_{o c}\right)$ of the cell.

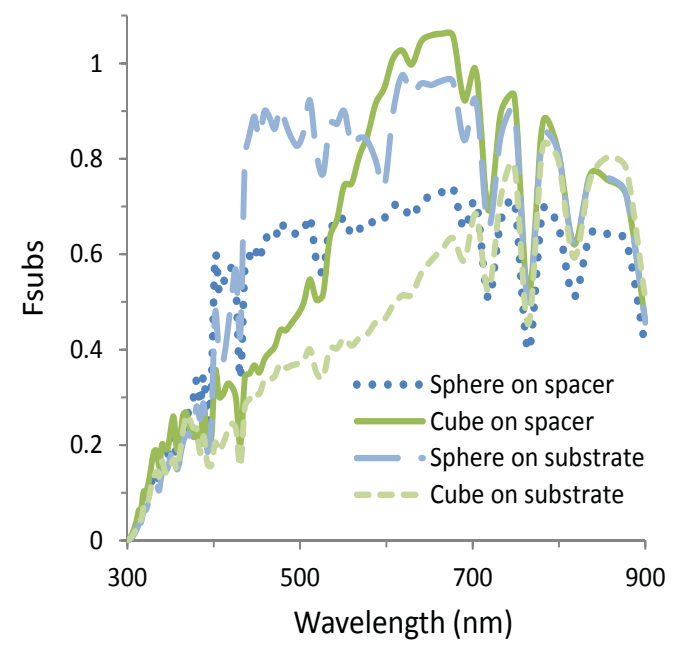

Figure 4.6: Fraction of light scattered forward into the substrate $\left(F_{\text {subs }}\right)$ by $150 \mathrm{~nm}$ $\mathrm{Ag}$ MNPs and calibrated to the power available in the AM1.5G spectrum at each wavelength. The $\mathrm{SiO}_{2}$ spacer layer is set to the optimized size of $25 \mathrm{~nm}$.

We now determine the effect of this optimal $25 \mathrm{~nm} \mathrm{SiO}_{2}$ spacer layer on the light scattering ability of the MNPs to scatter forwards into the substrate when compared directly to the AM1.5G solar spectrum. Fig. 4.6 shows the fraction of light coupled into the substrate multiplied by the power available in the AM1.5G solar spectrum. Fig. 4.6 shows a large increase in $F_{\text {subs }}$ in the longer $500-800 \mathrm{~nm}$ wavelength range when placing a $25 \mathrm{~nm}$ dielectric spacer between $\mathrm{Ag}$ nanocubes and the substrate. The increase occurs mainly due to the reduction in near-field coupling to the substrate, which reduces backscatter, and the LSPR blue-shifts due to the lower RI spacer.

In contrast, placing the nanospheres $25 \mathrm{~nm}$ away from the substrate has a negative effect on performance. This is possibly due to the different parameters for optimization between nanocubes and nanospheres. As the MNP shape changes from spherical to cubic the polarizability (i.e. the relative tendency of the MNPs electron cloud to 
be distorted from its normal shape by the optical driving field) changes as well [54]. Therefore the optimal effective index that each particle requires will be different, necessitating a different spacer layer thickness for nanocubes and nanospheres. Simulations indicate that $150 \mathrm{~nm} \mathrm{Ag}$ nanocubes require at least $20-25 \mathrm{~nm}$ spacing from the substrate to achieve optimal performance, while nanospheres require no more than $10 \mathrm{~nm}$.

Integrating under the curves, as a metric of relative performance, the $150 \mathrm{~nm} \mathrm{Ag}$ nanocubes placed on a thin $25 \mathrm{~nm} \mathrm{SiO}_{2}$ spacer layer show a $36.4 \%$ increase in forward scattered light compared to nanocubes sitting on the bare substrate. Unfortunately this is still a $12.3 \%$ decrease from the amount of light scattered forward by the reference case, where the Ag nanospheres are sitting directly on the bare a-Si substrate. Thus Ag nanocubes on an optimized spacer layer are still inferior to the perfect Ag nanospheres and another method is needed to bring nanocubes to their full potential for light absorption enhancement in thin-film a-Si solar cells.

\subsection{Effect of MNP material on plasmonic enhance- ment}

One possible solution to further optimize the MNP absorption and scattering properties is by blue-shifting the LSPR further and using off-resonant enhancement, instead of on-resonance enhancement. Changing the MNP material to Al, a nonresonant material with LSPRs that mainly lie outside of the solar spectrum such that they cannot couple directly to sunlight, shows significantly reduced particle absorption at short wavelengths [29], as well as a clear increase in forward scattering in this wavelength region [33].

The increase in forward scattering for nonresonant materials is due to the higher 
density of conduction electrons, resulting in an extremely negative $\epsilon_{m}$ which is desirable for the forward scattering configuration [33]. Whether or not the forward scattered light contributes to an increase, or reduction, generated photocurrent depends on the phase difference between the light transmitted across the a-Si interface, and that scattered by the MNPs [53]. Lim et al. [18] showed that below the LSPR wavelength, a phase shift in the polarizability of the MNPs causes the transmitted and scattered light to destructively interfere, resulting in a reduction in light absorption. Correspondingly, at wavelengths longer than the plasmon resonance light undergoes constructive interference leading to enhancements in light absorption. Since Al LSPRs lie in the UV region, the light scattered by Al MNPs must undergo constructive interference over the majority of the solar spectrum. In addition, Tsai et al. [53] demonstrated that $\mathrm{Al}$ MNPs reduce the phase difference in the transmitted and scattered light compared with Ag, thus leading to strong enhancements in both the VIS and near-IR regions, as opposed to just the near-IR for Ag particles.

Fig. 4.7 shows the results of 3D FDTD simulations for a simple model of a-Si as the substrate and $25 \mathrm{~nm}$ of $\mathrm{SiO}_{2}$ as the dielectric spacer layer. The results of the simulations have been calibrated to the AM1.5G spectrum in order to determine the exact behaviour of the MNPs under solar radiation, as in a practical application. Nanocubes of either material significantly outperform the traditional spherical MNP shape in terms of $Q_{\text {scat }}$ at longer wavelengths, and come close to matching nanospheres at the shorter wavelengths where solar radiation is most intense. In Fig. 4.7a the change of material from $\mathrm{Ag}$ to $\mathrm{Al}$ has a drastic effect on the scattering cross-sections of the MNPs. Despite the fact that Ag cubes appear to have a greater overall scattering cross-section when compared directly against the power in the solar spectrum, it is important to note that the scattering cross-section does not give the full picture in 


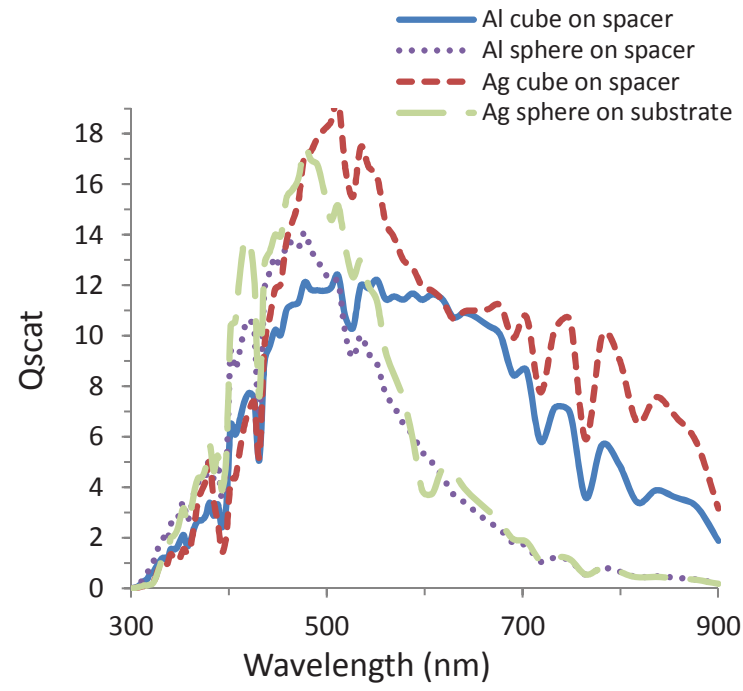

a)

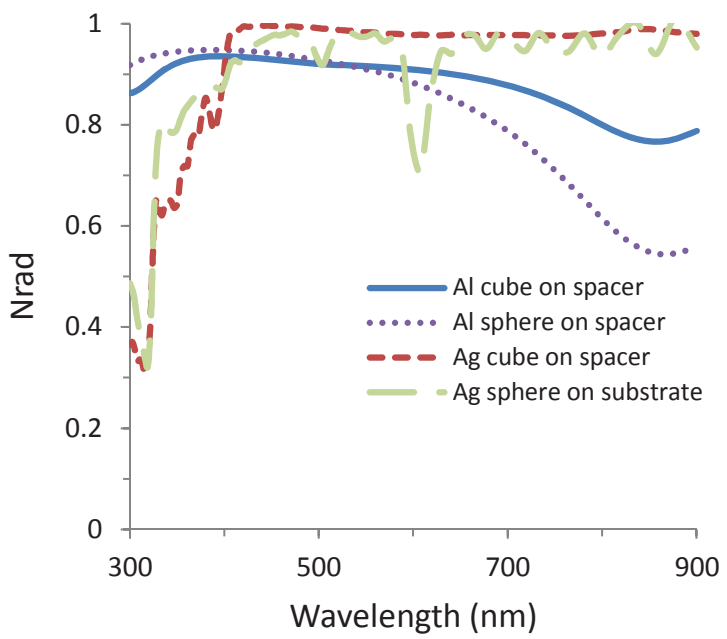

c)

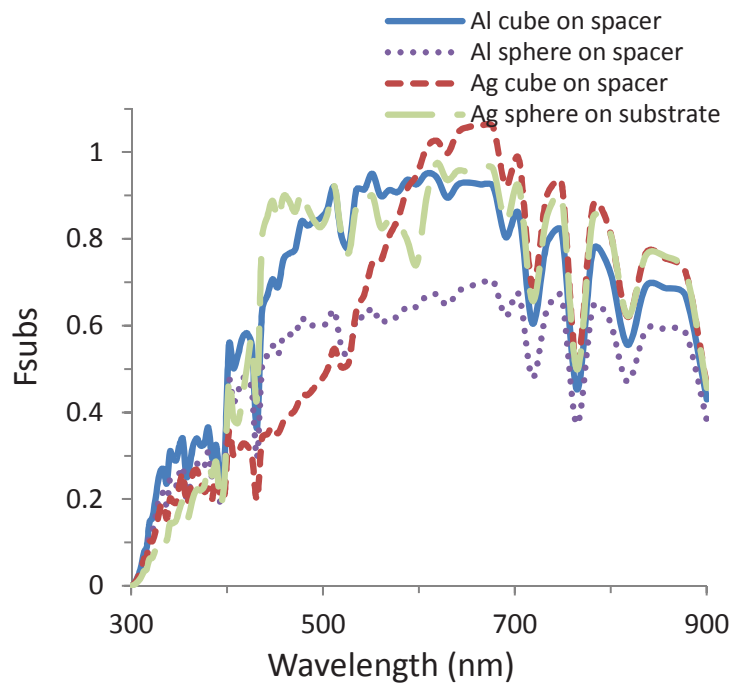

b)

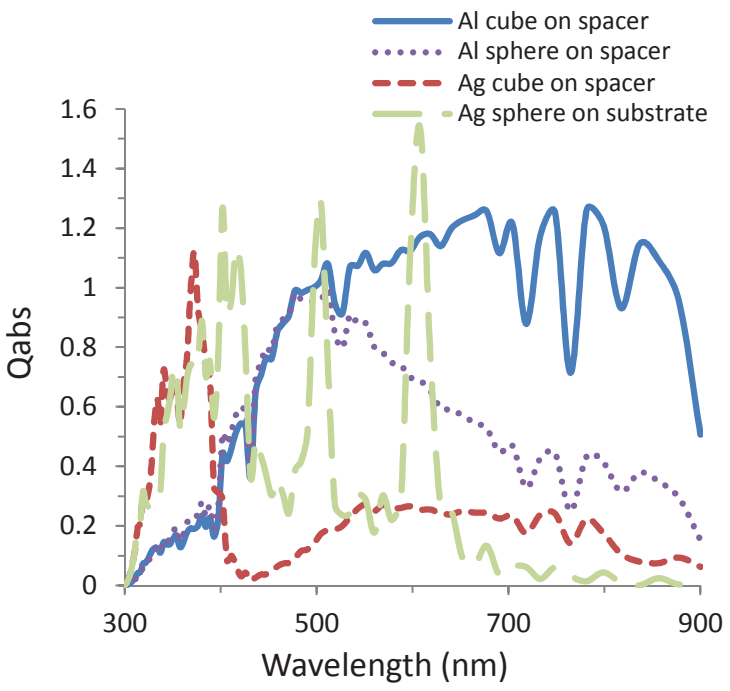

d)

Figure 4.7: (a) Normalized scattering cross-sections $\left(Q_{\text {scat }}\right)$. (b) Fraction of incident radiation forward scattered into the substrate $\left(F_{\text {subs }}\right)$. (c) Radiative efficiency of the particles $\left(N_{\text {rad }}\right)$. (d) Normalized absorption cross sections $\left(Q_{\text {abs }}\right)$. The spacer thickness is set to $25 \mathrm{~nm}$ and all quantities are calibrated to the power available in the AM1.5G spectrum at each wavelength.

terms of increasing the amount of light trapped and absorbed in a thin-film solar cell application. Scattering can be forwards, or backwards, with the latter being detrimental to cell performance. 
Fig. 4.7b depicts the actual fraction of incoming light that is scattered forward into the absorbing substrate $\left(F_{\text {subs }}\right)$ for $\mathrm{Al}$ and $\mathrm{Ag}$ nanocubes and $\mathrm{Al}$ nanospheres on a $25 \mathrm{~nm} \mathrm{SiO}_{2}$ dielectric spacer layer, as well as a reference Ag nanosphere sitting directly on the a-Si substrate. Given the 25nm $\mathrm{SiO}_{2}$ thickness, Al nanocubes show superior performance over all other geometries and materials in the majority of the UV-VIS spectrum. The Al nanocubes significantly improve the plasmonic characteristics in the short $(300-550 \mathrm{~nm})$ wavelength region while maintaining an enhancement in longer wavelength region. If we again integrate the area under the $F_{\text {subs }}$ curves in Fig. 4.7b from $300-900 \mathrm{~nm}$ and use this as a metric for the total amount of light scattered forward, the $\mathrm{Al}$ nanocubes show a $7.9 \%$ increase over the $\mathrm{Ag}$ nanocubes and a $1.1 \%$ decrease over the reference Ag spheres. Most of the superior performance of nanospheres occurs in the $700-900 \mathrm{~nm}$ wavelength range which is greater than the bandgap of a-Si.

If, in Fig. 4.7b we focus on the wavelength range less than the bandgap of a-Si, $\sim 1.7 \mathrm{eV}$ or $730 \mathrm{~nm}$, where a-Si absorbs light $\sim 100 \times$ faster than crystalline silicon wafers, the $\mathrm{Al}$ nanocubes scatter $16.7 \%$ more light into the a-Si absorber than the Ag nanocubes and 2.3\% more than the reference Ag nanospheres. This shows that Ag may be a better candidate for conventional crystalline solar cells as much of the enhancements from $\mathrm{Ag}$ are found in the near-IR region $[24,25]$ not relevant to a$\mathrm{Si}$; but very important for traditional crystalline solar cells. Comparing the results above to an $\mathrm{Al}$ nanosphere in the same configuration, the $\mathrm{Al}$ nanospheres lead to a $24.2 \%$ decrease in forward scattered light over the reference case, or, when considering the full $300-900 \mathrm{~nm}$ range, a $23.9 \%$ decrease in forward scattering showing that $\mathrm{Al}$ nanospheres tend to perform better in the long wavelength range but still offer no benefits over Ag nanospheres.

Fig. $4.7 \mathrm{c}$ gives the radiative efficiency of the MNPs, which measures how much 
of the total plasmonic extinction is due to scattering compared to absorption in the MNPs, and is defined as: $N_{\text {rad }}=\frac{Q_{\text {scat }}}{Q_{\text {scat }}+Q_{\mathrm{abs}}}$. Generally this reveals the minimum absorption that can be expected from an array of optimally placed particles [42]. Here, along with Fig. 4.7d we can see part of the reason for the poor performance of $\mathrm{Al} \mathrm{MNPs}$ at longer wavelengths. The $\mathrm{Al}$ nanoparticles have increased absorption cross-sections leading to a lower $N_{\text {rad }}$. However, because of the high dielectric constant of $\mathrm{Al}$ there is very little electric field penetration into the MNP, resulting in low overall absorption rates compared to silver nanoparticles [30], despite the increase in absorption cross-section.

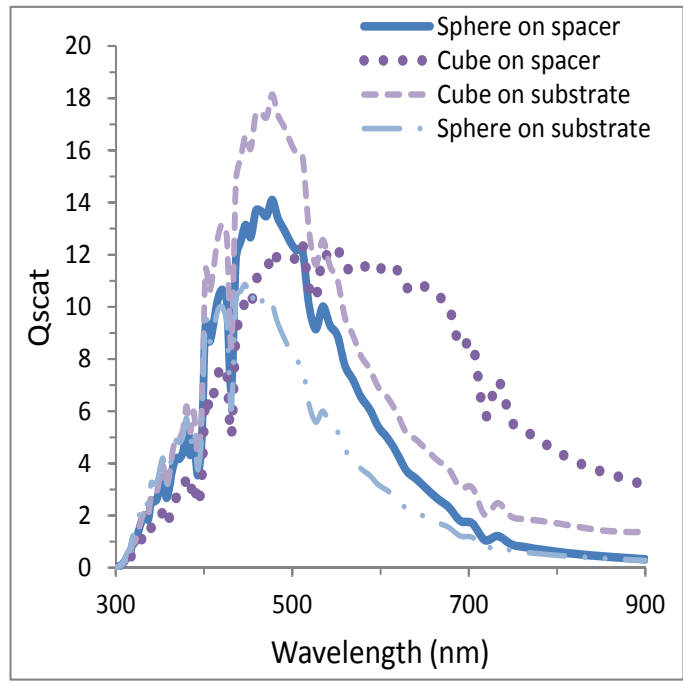

Figure 4.8: Normalized scattering cross-section $\left(Q_{\text {scat }}\right)$ (calibrated to the power available in the AM1.5G spectrum) of $150 \mathrm{~nm}$ Al MNPs sitting directly on a-Si substrate or a $25 \mathrm{~nm} \mathrm{SiO}_{2}$ spacer layer.

The trade-off made by using an off-resonant material such as $\mathrm{Al}$, is that $Q_{\text {scat }}$ and $F_{\text {subs }}$ are decreased in the longer wavelength ranges compared to $\mathrm{Ag}$, partly due to the increased absorption cross-section in the $450-900 \mathrm{~nm}$ range (see Fig. 4.7d), but mostly because the largest enhancements in light trapping are found nearer to the plasmon resonances. This is why most works have previously focused on red-shifting 
the LSPRs into the near-IR region to increase light absorption in that region, as explained in the introduction. However, Villesen et al. [55] showed experimentally that there is photocurrent enhancement only at frequencies lower than the main LSPR frequency (i.e. longer wavelengths). Hence the bluer LSPR positions of the Al MNPs allow enhancement to occur over a larger portion of the solar spectrum as opposed to Ag MNPs.

Up to the a-Si bandgap of $\sim 1.7 \mathrm{eV}$ Al MNPs perform very well showing an almost constant $N_{\text {rad }}$ close to $90 \%$ and achieving improvements over the Ag MNPs in the $300-400 \mathrm{~nm}$ range. Fig. $4.7 \mathrm{~d}$ shows that the scaled absorption cross-section remains higher for Al MNPs but the increased $F_{\text {subs }}$ implies that the increased $Q_{\text {scat }}$ outweighs this. The Al MNPs also have an absorption cross-section $3 \times$ less than Ag MNPs in the $300-450 \mathrm{~nm}$ range which further illustrates the trade-off made when using Al MNPs, increased plasmonic enhancement up to $550 \mathrm{~nm}$ and slightly decreased enhancement at longer wavelengths compared to Ag MNPs. For a-Si solar cells this makes Al an almost ideal metal as the bandgap occurs just past the point where Ag outperforms $\mathrm{Al}$.

In contrast to nanocubes, $\mathrm{Al}$ nanospheres perform poorly, especially at longer wavelengths due to their initially lower $Q_{\text {scat }}$ in that region. Fig. 4.2 shows the weak $Q_{\text {scat }}$ for $\mathrm{Ag}$ nanospheres compared to $\mathrm{Ag}$ nanocubes in the $550-900 \mathrm{~nm}$ range. Changing the nanosphere material to $\mathrm{Al}$, as in Fig. 4.8, further reduces the scattering ability of the nanospheres at longer wavelengths due to the blue-shifting of the LSPRs. Switching entirely to Al we can compare the performance of Al nanospheres and nanocubes directly on a substrate and again on a $25 \mathrm{~nm} \mathrm{SiO}_{2}$ spacer layer. Although the optimal spacer layer thickness for Al MNPs is slightly different than the $25 \mathrm{~nm}$ obtained for Ag MNPs (Fig. 4.5) we chose to use a consistent thickness to investigate the effect of the spacer layer scattering environment. The Al nanocubes 
on the dielectric spacer layer provide an exceptionally broadband scattering profile across most of the relevant spectrum.

The results in Fig. 4.8 have been calibrated to the power available at each wavelength in the AM1.5G spectrum in order to directly compare the scattering peaks with the solar spectrum peaks. Doing this shows that nanocubes maintain their broadband scattering in the relevant solar wavelengths. In comparison $\mathrm{Al}$ nanospheres or even nanocubes directly on the substrate have very narrow scattering peaks, although these are clearly in the peak of the solar spectrum as the scaled $Q_{\text {scat }}$ shows a large peak around $500 \mathrm{~nm}$ for these configurations, a broadband scattering much more desirable for solar cell light absorption. Thus Fig. 4.8 demonstrates that the use of a spacer layer is critical for plasmonic enhancement when using Al MNPs.

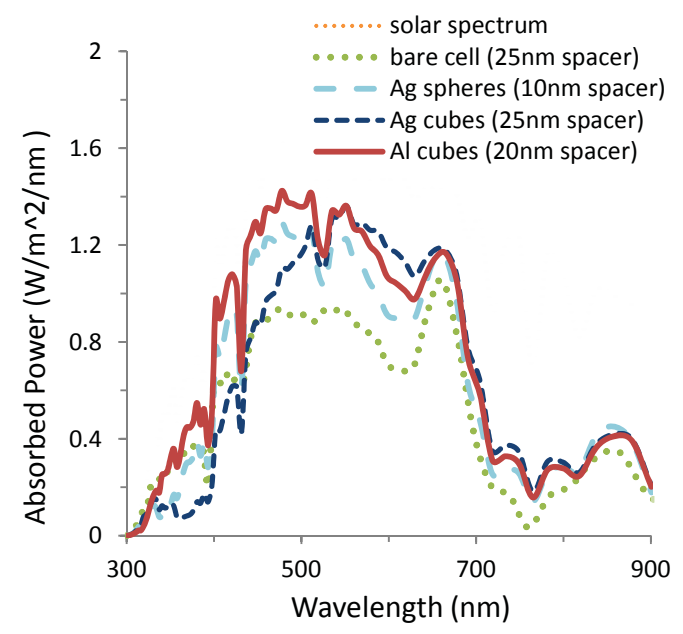

Figure 4.9: Power absorbed per $\mathrm{m}^{2} / \mathrm{nm}$ in a 240nm a-Si layer with a $25 \%$ surface coverage of uniformly spaced 80nm nanoparticles on optimized $\mathrm{SiO}_{2}$ spacer layers

By combining all our findings together, we discover that 80nm MNPs are the optimal size for all nanocubes and nanospheres to provide the greatest enhancement in absorbed power in a $240 \mathrm{~nm}$ a-Si solar cell. Spacer layers are individually optimized 
under each MNP configuration. For simplicity of analysis we assume a uniform distribution of the $80 \mathrm{~nm}$ MNPs with a $25 \%$ surface coverage as periodicity effects have been shown to contribute to only a marginal increase in absorbed power [53]. We also switch now to a 2D simulation for the realizable solar cell geometries as computer memory constraints limit the possibility for accurate 3D simulation. Fig. 4.9 shows the simulation results which have been normalized to the solar spectrum to allow the absorbed power per solar wavelength to be determined. Here we see that at a $25 \%$ surface coverage on individually optimized spacer layers all MNPs lead to more absorbed power than the bare cell (25nm spacer layer); but, the Al nanocubes $(20 \mathrm{~nm}$ spacer) outperform Ag nanocubes (25nm spacer) and Ag nanospheres (10 $\mathrm{nm}$ spacer) up to $550 \mathrm{~nm}$ wavelength.

At longer wavelengths the Ag nanocubes induce slightly larger enhancements; but, overall $\mathrm{Al}$ nanocubes allow extraction of the most power from the incoming solar radiation. This leads us to conclude that in order to take advantage of the increased forward scattering and enhancements at wavelengths in the near-IR, Ag nanoparticles may be best suited to conventional bulk c-Si wafers. Fig. 4.9 also clearly shows the effect of the blue-shifted cross-over point from reductions to enhancements where the Al nanocubes show enhancement in absorbed power over the bare cell for wavelengths greater than $350 \mathrm{~nm}$, whereas the Ag MNPs only begin to show enhancement past $400 \mathrm{~nm}$ and $450 \mathrm{~nm}$ for nanospheres and nanocubes respectively.

If we integrate under the curves up to the $730 \mathrm{~nm}$ bandgap of a-Si, we can find the total power absorbed by the active a-Si layer and compare to the reference case. The bare reference cell with a $25 \mathrm{~nm} \mathrm{SiO}_{2}$ layer absorbs $\sim 54.8 \%$ of the available incoming solar spectrum in the $300-730 \mathrm{~nm}$ range. The Ag nanospheres and nanocubes allow the a-Si to absorb $66.9 \%$ and $66.8 \%$ respectively, while the $\mathrm{Al}$ nanocubes encourage a full $76.1 \%$ absorption of the incoming solar radiation. This is a $13.8 \%$ increase over 
the optimized Ag nanosphere case and a 38.9\% increase in absorbed power compared to the bare reference cell.

Hence for a-Si thin-film solar cells the ideal plasmonic nanoparticle for light trapping is the $\mathrm{Al}$ nanocubes as they retain high $Q_{\text {scat }}$ throughout the solar spectrum providing strong enhancement in power absorbed in the cell as seen in Fig. 4.9.

\subsection{MNP Optimizations}

Many factors determine how effective an array of plasmonic MNPs will be at enhancing the light absorption and photocurrent generation in a thin-film solar cell. The wavelength position of a localized surface plasmon resonance at the metal/dielectric interface is of utmost importance and a driving factor in determining where and how solar cell enhancement occurs. The plasmon resonance wavelength is affected by a highly complex parameter space and can be tuned accordingly by adjusting the MNP material, dielectric environment, array pitch (surface coverage) and MNP dimensions. Varying any of these parameters allows large changes in resonance position and localized surface plasmon behaviour, however it should be noted that it is much easier to red-shift the resonance position than it is to blue-shift the resonance while still achieving good forward scattering of light and thus, photocurrent enhancement.

Before beginning experiments and actual fabrication of MNP enhanced thin-film solar cells we attempt to optimize the MNP performance in terms of all of the parameters in this complex parameter space. It was shown in earlier sections that $80 \mathrm{~nm}$ $\mathrm{Al}$ cubes were the best in terms of MNP dimensions and material. The remaining aspects to be optimized exist in a two dimensional space consisting of the array pitch (surface coverage) and dielectric environment.

The previous sections examined MNPs on a $\mathrm{SiO}_{2}$ spacer layer $\left(n_{\text {space }}=1.45\right)$. This 
low refractive index leads to a significant blue-shift in plasmon resonance frequency and allowed a large portion of incident light to be forward scattered over a large wavelength band in the solar spectrum. In a-Si solar cells a TCO is usually needed for efficient current collection due to the relatively poor lateral conductivity of a-Si. Unfortunately, $\mathrm{SiO}_{2}$ is an insulator and a poor choice for practical a-Si solar cells. The low index TCO, ITO $\left(n_{\text {space }}=1.9\right)$, is usually used to help collect current and deliver it to the ohmic contacts. The slightly higher refractive index of ITO demands a separate optimization and also leads to more backscattering which will produce a

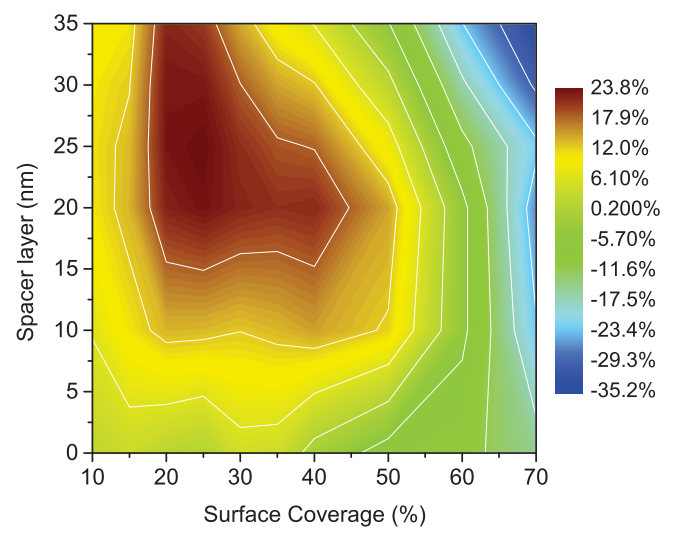

(a)

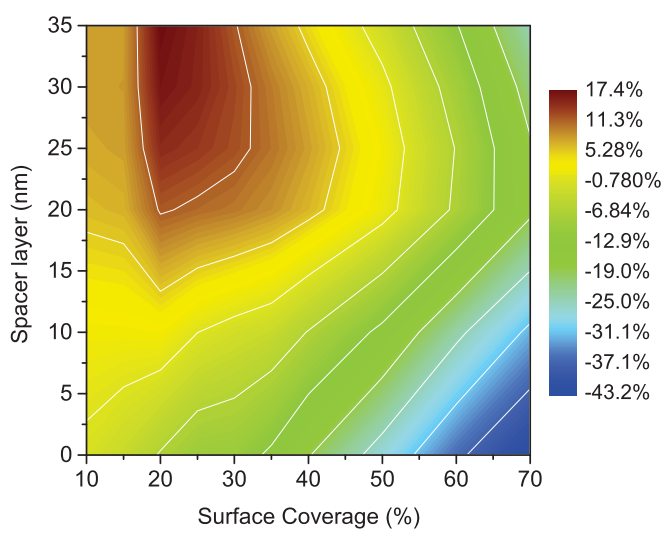

(b)

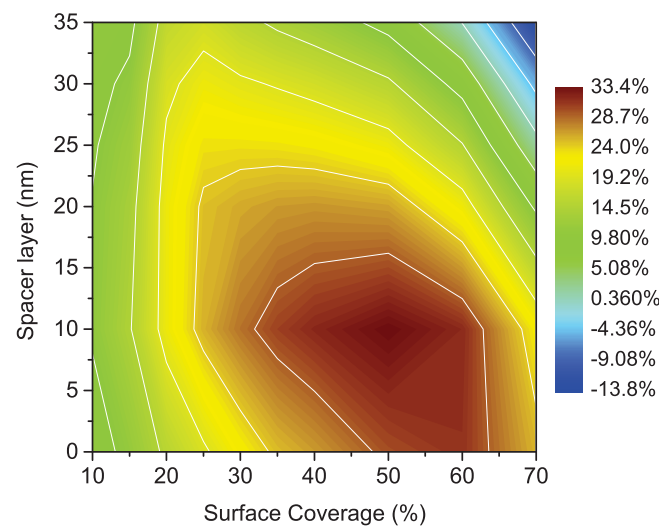

(c)

Figure 4.10: Contour optimization of 80 $\mathrm{nm} \mathrm{Ag} \mathrm{a)} \mathrm{nanocube,} \mathrm{b)} \mathrm{nanohemisphere}$ and c) nanosphere surface coverage and $\mathrm{SIO}_{2}$ spacer layer thickness 
lower enhancement compared to the $\mathrm{SiO}_{2}$ spacer layer.

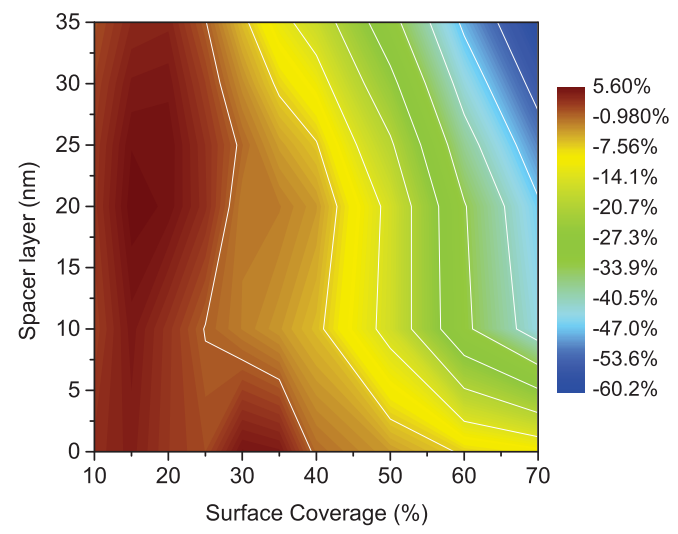

(a)

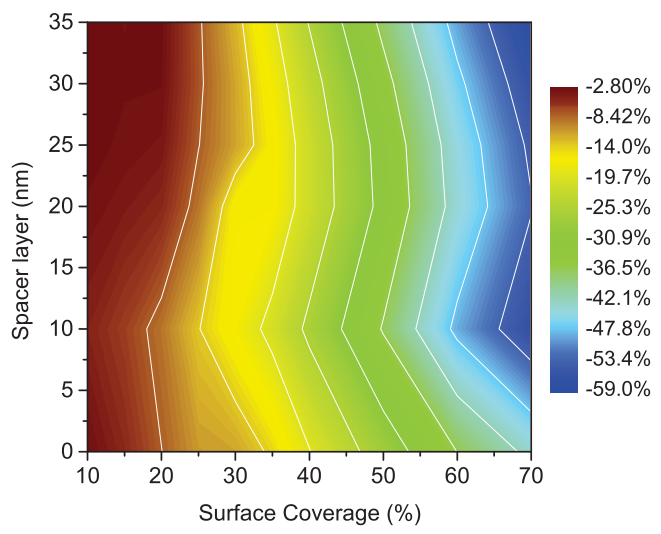

(b)



(c)

Figure 4.11: Contour optimization of 80 $\mathrm{nm} \mathrm{Ag} \mathrm{a)} \mathrm{nanocube,} \mathrm{b)} \mathrm{nanohemisphere}$ and c) nanosphere surface coverage and ITO spacer layer thickness

Optical numerical simulations were conducted with Lumerical FDTD Solutions on the structure of Fig. 5.1 for both $\mathrm{SiO}_{2}$ and ITO spacer layers. A simulation was conducted at every minor grid point and the results plotted to produce the contour plots shown in Figs. 4.10, 4.11, 4.12 and 4.13. The results are indicated in terms of short circuit current $\left(J_{\mathrm{sc}}\right)$ enhancement over a similar solar cell structure with 


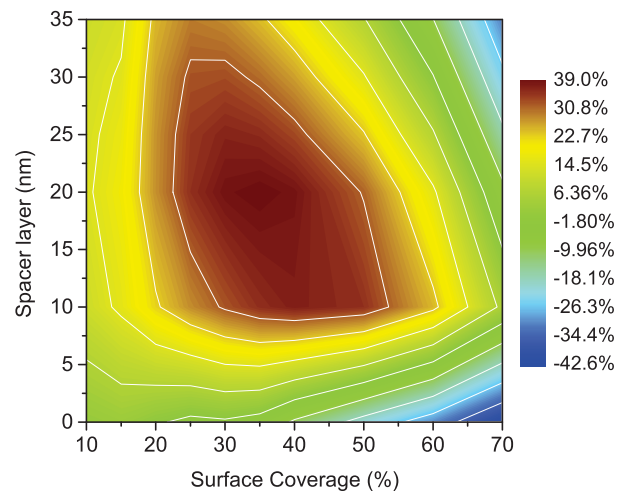

(a)



(b)

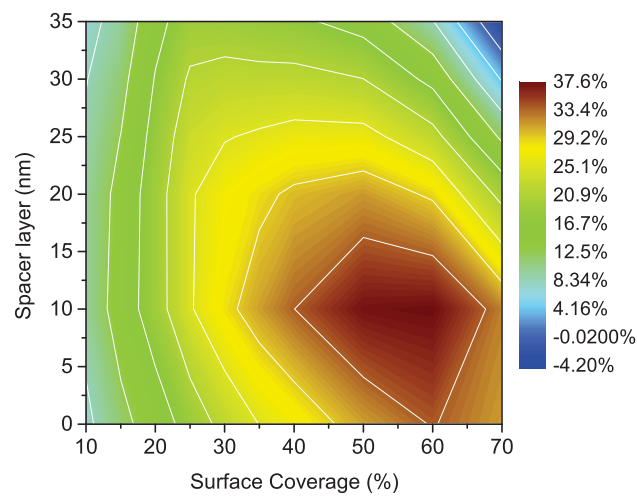

(c)

Figure 4.12: Contour optimization of $80 \mathrm{~nm} \mathrm{Al} \mathrm{a)} \mathrm{nanocube,} \mathrm{b)} \mathrm{nanohemisphere}$ and c) nanosphere surface coverage and $\mathrm{SiO}_{2}$ spacer layer thickness

no MNPs integrated on the top surface. $J_{\text {sc }}$ was calculated assuming all absorbed photons are collected and contribute to the photocurrent.

Optimizations were performed for 80 $\mathrm{nm}$ diameter $\mathrm{Ag}$ and $\mathrm{Al}$ cubes, spheres and hemispheres on $\mathrm{SiO}_{2}$ and ITO spacer layers so that relevant comparisons of enhancements could be made for the most common shapes and plasmonic materials. Fig. 4.10 confirms the optimum parameters for Ag spheres and cubes used in the previous sections, approximately a $25 \%$ surface coverage on a $20-25 \mathrm{~nm} \mathrm{SiO}_{2}$ spacer layer for cubes and a $60 \%$ surface coverage on a $5-10 \mathrm{~nm}$ spacer layer for spheres. Hemispheres show a notably lower enhancement than either cubes or spheres, we postulate this is 


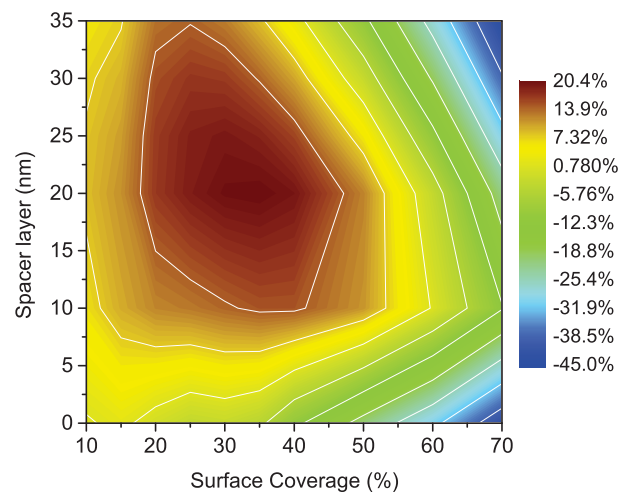

(a)

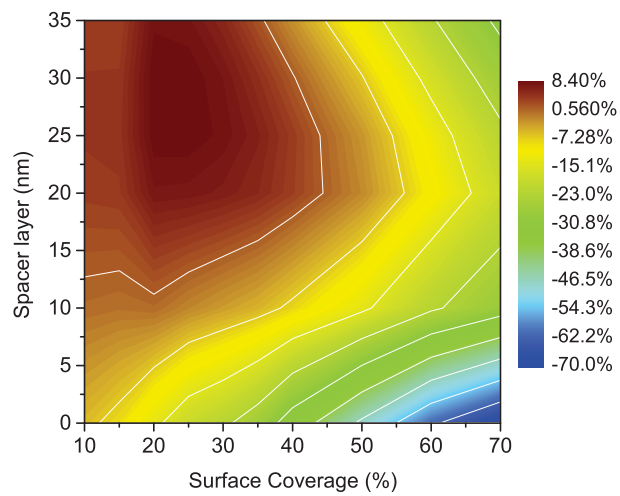

(b)

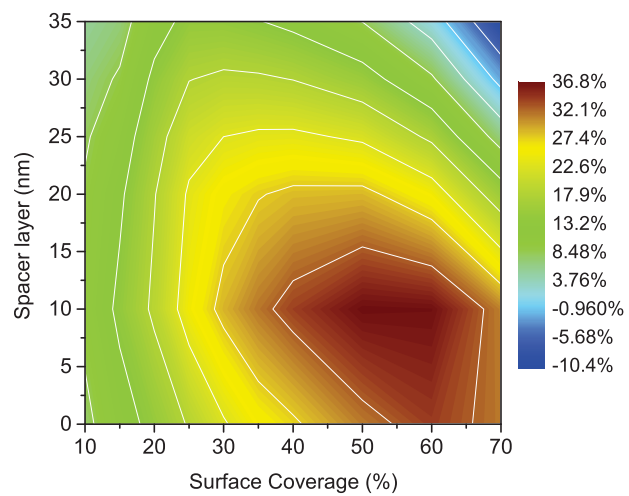

(c)

Figure 4.13: Contour optimization of $80 \mathrm{~nm} \mathrm{Al} \mathrm{a)} \mathrm{nanocube,} \mathrm{b)} \mathrm{nanohemisphere}$ and c) nanosphere surface coverage and ITO spacer layer thickness

due to a combination of the worst features of both spheres and cubes (i.e the lower scattering efficiency of the spherical shape, and the increased backscattering due to the large contact area with the substrate).

Switching over to a higher index ITO spacer layer, absolute $J_{\text {sc }}$ is reduced by $13.4 \%$ and $13 \%$ for Ag cubes and hemispheres respectively. This results in the $76 \%$ and $116 \%$ reductions in $J_{s c}$ enhancement noticed between Figures 4.10 and 4.11. Ag spheres show only a slight decrease of $1.6 \%$ compared to the $\mathrm{SiO}_{2}$ spacer as a result of low contact area to the spacer. For large enhancements Ag spheres require extremely high surface coverages, which are not practical to fabricate even with colloidal solutions 
Table 4.1: Optimal parameters for $J_{s c}$ enhancement in a-Si solar cells

\begin{tabular}{cccc}
\hline Shape (Material) & ITO Spacer layer & Surface Coverage & $J_{s c}$ Enhancement \\
\hline \hline Cubes (Ag) & $20 \mathrm{~nm}$ & $15 \%$ & $5.6 \%$ \\
Cubes (Al) & $\mathbf{2 0 n m}$ & $\mathbf{3 5 \%}$ & $\mathbf{2 0 . 4 \%}$ \\
Hemispheres (Ag) & $30 \mathrm{~nm}$ & $10 \%$ & $-2.8 \%$ \\
Hemispheres (Al) & $30 \mathrm{~nm}$ & $20 \%$ & $8.4 \%$ \\
Spheres (Ag) & $0 \mathrm{~nm}$ & $60 \%$ & $30.8 \%$ \\
Spheres (Al) & $10 \mathrm{~nm}$ & $50 \%$ & $36.6 \%$ \\
\hline
\end{tabular}

due to particle aggregation.

The next optimization is done for $\mathrm{Al}$ nanoparticles on $\mathrm{SiO}_{2}$ in Fig. 4.12. This clearly shows that $\mathrm{Al}$ nanocubes now outperform even spheres providing a $1.4 \%$ absolute increase in enhancement. The extremely favourable conditions that a low index spacer layer provides, combined with the UV located plasmon resonance of $\mathrm{Al}$ and the high scattering cross-section of nanocubes result in very large enhancement of $39 \%$ for Al nanocubes on a thin $20 \mathrm{~nm} \mathrm{SiO}_{2}$ spacer at $30-35 \%$ surface coverage. Al hemispheres also perform admirably if optimized correctly. Again the ideal surface coverage is quite high, around $40-50 \%$, however, these are easier to fabricate than perfect spheres since metal island annealing can provide quite high surface coverages.

Finally, Al MNPs are optimized on an ITO spacer layer for implementation in fabrication. Fig. 4.13 shows that the $\mathrm{Al}$ nanocubes should give rise to a $20.4 \% J_{\mathrm{sc}}$ enhancement compared with hemispheres at only $9 \%$. This constitutes a $5.4 \%$ and $4.8 \%$ drop in $J_{s c}$ from the ideal case on $\mathrm{SiO}_{2}$ for cubes and hemispheres respectively. Again perfect spheres demonstrate almost no reduction after placement on ITO, but, fabrication is extremely challenging at the $60 \%$ surface coverage needed. Fabrication of arrays of perfect spheres is very difficult no matter the array pitch desired. Spheres 
cannot be formed via e-beam lithography, and thus any large area techniques such as nano-imprint lithography would also prove non-viable. This makes the cubic shape much more desirable to work with in terms of mass production and fabrication. The optimal parameters for Al nanocubes are a $20 \mathrm{~nm}$ ITO spacer layer at $25-35 \%$ surface coverage, indicating a fairly robust and fabrication tolerant system as long as the spacer layer is of accurate thickness.

\subsection{Investigation of an optimal ITO spacer layer}

We examine more closely the situation when a MNP sits on a thin indium-doped tin oxide (ITO) film (see inset Fig. $4.14 \mathrm{~b})$, where $n_{\text {film(ITO) }}=1.9$ and $n_{\text {subs(a-Si) }}=4.23$. The film and substrate material models used are simple, real refractive indices such that the dispersion effects of the material itself are neglected. Given that ITO is a widely used transparent front contact we determine the effect of a thin ITO layer on the performance of plasmonic nanocubes and optimize the nanocube parameters accordingly.

Fig. 4.14 shows the results of 3D FDTD simulations for $\mathrm{Ag}$ and $\mathrm{Al}$ nanocubes on a $25 \mathrm{~nm}$ ITO spacer. In the case of an ITO spacer the Al nanocubes have larger $Q_{\text {scat }}$ than $\mathrm{Ag}$ nanocubes over most of the solar spectrum, however, a larger $Q_{\text {scat }}$ is not the end of the story. Scattering can be in the forward or backwards direction, with the latter hurting solar cell performance. $F_{\text {subs }}$ gives the fraction of light which is scattered forward, out of the total light scattered in all directions by the NP. If we compare the $Q_{\text {scat }}$ with $F_{\text {subs }}$ for $\mathrm{Ag}$ nanocubes in Fig. $4.14 \mathrm{a}$, we can deduce that a lot of the scattering occurring in the $350-500 \mathrm{~nm}$ range is back-scattering as opposed to the beneficial forward scattering. On the other hand Fig. 4.14b shows 


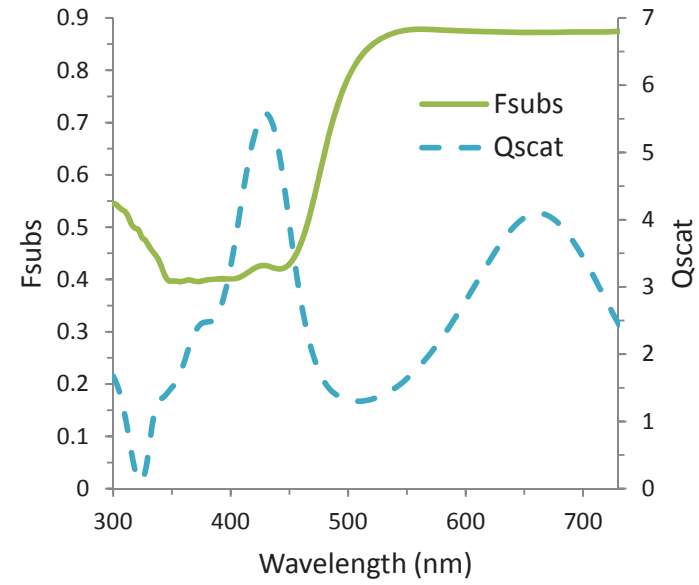

(a)

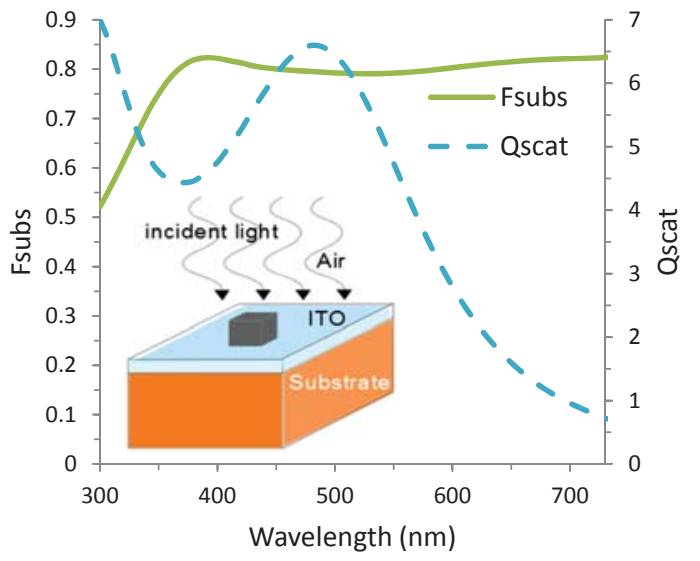

(b)

Figure 4.14: Normalized scattering cross-section $\left(Q_{\text {scat }}\right)$ compared to fraction of forward scattered light $\left(F_{\text {subs }}\right)$ for $80 \mathrm{~nm}$ a) $\mathrm{Ag}$ and b) Al nanocubes on a $25 \mathrm{~nm}$ ITO spacer layer

that much more of the scattering is in the forward direction, especially around $500 \mathrm{~nm}$ wavelength, which is important because the peak of the solar spectrum occurs in this region. Thus, when comparing NP material performance directly to AM1.5G, the Al MNPs have an advantage over the Ag MNPs.

Aluminium's much higher density of conduction electrons, resulting in an extremely negative $\epsilon_{m}$, which is desirable for the forward scattering configuration [33], allows the $\mathrm{Al}$ nanocubes to direct much more of the incoming radiation into the solar cell than Ag nanocubes. Integrating to find total area under the curves up to the band-gap of a-Si, we see that $\mathrm{Al}$ nanocubes provide $14.6 \%$ more forward scattering than Ag nanocubes and have a $54 \%$ higher total integrated $Q_{\text {scat }}$.

We also simulate a practical $240 \mathrm{~nm}$ a-Si solar cell with MNPs on a 25nm ITO spacer layer at a $25 \%$ particle surface coverage to determine the total increase in absorption inside the active layer due to path length enhancement by the nanocubes. Fig. 4.15 shows the absorption rate in the a-Si scaled by the power available in AM1.5G to get the total solar power absorbed, and demonstrates the trade-off made 
by using a nonresonant material such as Al. For such materials, $Q_{\text {scat }}$ and $F_{\text {subs }}$ are decreased in the longer wavelength ranges compared to $\mathrm{Ag}$ (seen in Fig. 4.14) because the largest enhancements in light trapping are found nearer to the plasmon resonances. This is why most works have previously focused on red-shifting the plasmon resonances into the near-IR region since light here is poorly absorbed. However, Villesen et al. [55] showed experimentally that there is photocurrent enhancement only at frequencies lower than the the main resonance frequency. Hence the resonance positions nearer to the UV, such as those of Al MNPs, allow enhancement to occur over a larger portion of the solar spectrum as opposed to Ag MNPs.

At wavelengths below 700nm, Fig. 4.15 shows that Al nanocubes encourage much more absorption in the active layer of the solar cell as opposed to the Ag MNPs. Only at longer wavelengths closer to the band-gap of a-Si do the Ag MNPs even start to outperform the bare cell or the Al MNPs. When comparing the absorption spectra in Fig. 4.15 it is clear that the $\mathrm{Al}$ MNPs show a large increase in absorbed power right at the peak of the incoming solar radiation which allows the underlying cell to be more efficient. Focusing on the wavelength range up to the band-gap of a-Si, $730 \mathrm{~nm}$, the $\mathrm{Al}$ nanocubes increase absorption in the active layer by $24.3 \%$ compared to the $\mathrm{Ag}$ nanocubes and $19.4 \%$ compared to the bare reference cell with a $25 \mathrm{~nm}$ ITO spacer layer on top.

We have clearly shown that $\mathrm{Al}$ nanocubes are much better suited to practical a-Si thin-film cells due to their ability to increase forward scattering and decrease parasitic absorption compared to Ag MNPs, especially on an ITO electrode. We looked at nanocubes on a thin ITO top contact over the wavelength range less than the bandgap of a-Si. A forward scattering enhancement of $14.6 \%$ was observed with Al MNPs compared to Ag MNPs. Using a nonresonant metal (Al) comes at the cost 




Figure 4.15: Power absorbed per $\mathrm{m}^{2} / \mathrm{nm}$ in a $240 \mathrm{~nm}$ a-Si solar cell with optimal $80 \mathrm{~nm}$ MNPs integrated on the surface of a thin 20 $\mathrm{nm}$ ITO top contact, compared with a particle free cell and the power available in the AM1.5G solar spectrum.

of reduced enhancement around the poorly absorbed near-IR region; however, the benefits outweigh the costs for a high band-gap absorber such as a-Si which absorbs most efficiently in the blue-green region of the spectrum. A $25 \%$ uniform surface coverage of $80 \mathrm{~nm} \mathrm{Al}$ nanocubes on a thin ITO layer provided a $24.3 \%$ increase in absorbed power over Ag nanocubes, and a $19.4 \%$ increase in absorbed power over a basic, NP free cell.

\subsection{Detailed Examination of Nanocube Behaviour}

\subsubsection{Comparison to Traditional Anti-reflection Coatings}

Thin film a-Si solar cells require a TCO for efficient operation. ITO $\left(n_{\text {ITO }}=1.9\right)$ is a common TCO for a-Si solar cells and also serves as a convenient, dual purpose anti-reflection coating (ARC). According to the theory, an optimal ARC should be 
one quarter of a wavelength to ensure that waves reflected from the ARC top surface are out of phase with the wave reflected from the silicon surface. The out-of-phase reflected waves destructively interfere with one another, resulting in zero net reflected energy. Quantitatively the thickness is ideally

$$
\begin{aligned}
t & =\frac{\lambda_{0}}{4 n_{1}} \\
& =\frac{500 \mathrm{~nm}}{4 \times 1.9} \\
& =66 \mathrm{~nm}
\end{aligned}
$$

This indicates that the optimal ARC thickness, $t$, should lie around $66 \mathrm{~nm}$. A real ITO ARC was simulated using FDTD at thickness' up to $150 \mathrm{~nm}$ and it was found that $60 \mathrm{~nm}$ of ITO provided the largest short circuit current $\left(J_{\mathrm{sc}}\right)$ of $19.9 \mathrm{~mA} / \mathrm{cm}^{2}$. ARCs have limitations however in that they do not provide large angle scattering. Using Snell's Law, $n_{1} \sin \theta_{1}=n_{2} \sin \theta_{2}$ and $n_{\mathrm{ITO}}=1.9, n_{\mathrm{a}-\mathrm{Si}}=4.23$, if the light is incident at $\theta_{1}=45^{\circ}$ then light is refracted into the substrate at a maximum of $18.5^{\circ}$ with most rays being refracted at smaller angles resulting in little path length enhancement and no total internal reflection (TIR). Normally incident light is reflected at the back surface and then exits the cell after only 2 passes through the active layer.

On the other hand, investigating the angular scattering of Ag and Al MNPs on an a-Si substrate through FDTD leads to the angular scattering distribution shown in Fig. 4.16. These scattering distributions represent normally incident light being scattered into the far-field at a monitor placed 50 $\mathrm{nm}$ inside the a-Si substrate. The numbers $10,20,30,40,50$ on the plots correspond to the radially increasing concentric rings representing the angles at which light is scattered into the far-field. The intensity at a particular angle is represented on the colour scale. As we noted extensively in the 




ux

(a)

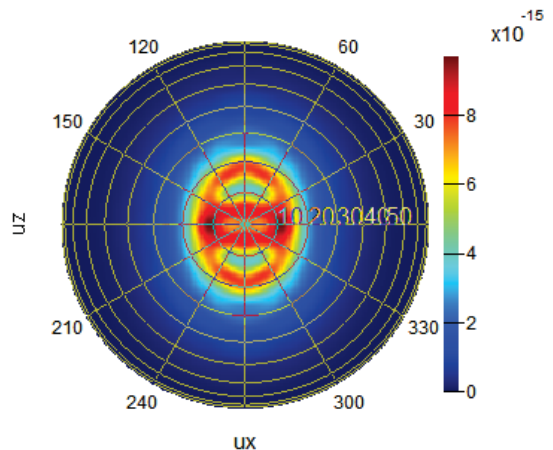

(c)

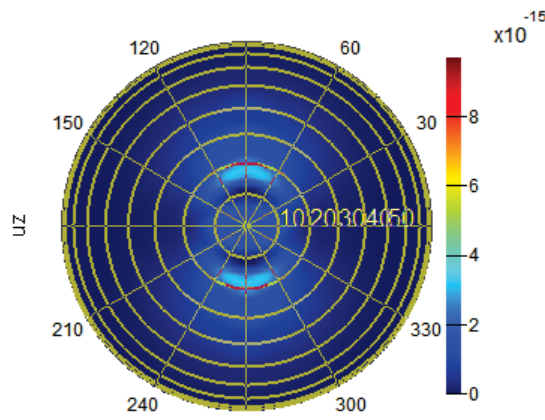

ux

(b)

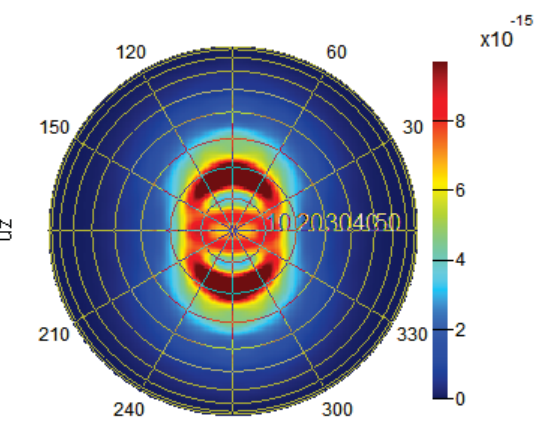

ux

(d)

Figure 4.16: Intensity of scattered light from $80 \mathrm{~nm} \mathrm{Ag}$ cubes on $20 \mathrm{~nm}$ of a) $\mathrm{SiO}_{2}$, b) ITO and $80 \mathrm{~nm} \mathrm{Al} \mathrm{cubes} \mathrm{on} 20 \mathrm{~nm}$ of c) $\mathrm{SiO}_{2}$ and d) ITO at $500 \mathrm{~nm}$

previous sections $(4.4,4.6)$, Ag MNPs scatter far less light forward into the substrate on $\mathrm{SiO}_{2}$ or ITO (Fig. 4.16b and 4.16a) compared to Al MNPs. Al MNPs have a higher intensity of scattered light at all angles compared with Ag MNPs, and on ITO only the Al cubes manage to scatter light at angles which exceed the critical angle at the a-Si/ITO interface of $26.7^{\circ}\left(\theta_{c}=\sin ^{-1}\left(\frac{1.9}{4.23}\right)\right)$. Thus even some normally incident light scattered by Al MNPs will remain trapped within the a-Si absorber layer via TIR.

In contrast, the scattering profile of the nanohemispheres in Fig. 4.17, on the same colour scale as Fig. 4.16, shows that, in comparison to nanocubes, the nanohemipsheres scatter hardly any light forward and definitely don't have the same large angle 




(a)



ux

(b)

Figure 4.17: Intensity of scattered light from 80nm a) Ag and b) Al nanohemispheres on $20 \mathrm{~nm}$ of ITO

scattering seen with the cubic MNPs. This lack of forward scattering at large angles is the main reason that nanohemispheres are not the best choice for light trapping in thin-film solar cells, providing only small enhancements in $J_{s c}$.

It is not prudent in the current situation to compare directly the short circuit currents achieved when ARCs or MNPs are used as light trapping techniques. In the optimizations of Section 4.5 only percentage increase over the same cell without MNPs was considered. This did not take into account the actual $J_{s c}$, as the combined ARC/MNP system will provide larger $J_{s c}$ over either method used separately. Of course increasing the spacer layer means that the spacer layer acts as a better ARC, however the MNP's ability to forward scatter light decreases past the optimal $25 \mathrm{~nm}$ spacer layer.

This is a subject for further optimization which has been partly completed in the case of a simple spacer layer, with no overlayer covering the particles, in Table 4.2. We again use the $240 \mathrm{~nm}$ solar cell with an $80 \mathrm{~nm}$ Al back contact and varying spacer layer thickness. Spacer thickness was increased until $J_{s c}$ reached a maximum value while still maintaining enhancement over the identical cell without MNPs. Table 4.2 
Table 4.2: Best achievable $J_{s c}$ showing increase over cell without MNPs

\begin{tabular}{|c|c|c|}
\hline $\begin{array}{l}\text { MNP shape } \\
\text { (Material) }\end{array}$ & $\begin{array}{c}\text { Spacer layer material } \\
(\text { Thickness }(n m))\end{array}$ & $J_{s c}\left(A / m^{2}\right)$ \\
\hline Cubes (Al) & $\mathrm{SiO}_{2}(25)$ & 210.6 \\
\hline Hemispheres (Al) & $\mathrm{SiO}_{2}(50)$ & 207.57 \\
\hline Spheres (Al) & ITO (45) & 204.3 \\
\hline Cubes (Al) & ITO (45) & 204.1 \\
\hline Spheres (Al) & $\mathrm{SiO}_{2}(10)$ & 202.89 \\
\hline Spheres (Ag) & $\operatorname{ITO}(40)$ & 201.25 \\
\hline Optimal ARC & ITO (60) & 199.0 \\
\hline Cubes (Ag) & $\mathrm{SiO}_{2}(35)$ & 197.2 \\
\hline Hemispheres (Al) & $\operatorname{ITO}(40)$ & 196.7 \\
\hline Hemispheres (Ag) & $\mathrm{SiO}_{2}(40)$ & 193.85 \\
\hline Spheres (Ag) & $\mathrm{SiO}_{2}(10)$ & 193.82 \\
\hline Cubes (Ag) & ITO $(40)$ & 188.8 \\
\hline Hemispheres (Ag) & ITO (35) & 172.49 \\
\hline
\end{tabular}

shows, in descending order, the maximum $J_{s c}$ obtainable using the simple spacer layer and allowing any surface coverage of the particles. In terms of absolute $J_{s c}$ the $\mathrm{Al}$ particles outperform the Ag particles in almost all cases, with the Al cubes leading to the largest possible $J_{s c}$ of $21.06 \mathrm{~mA} / \mathrm{cm}^{2}$.

Fig. 4.18 takes the best $J_{s c}$ regardless of surface coverage for the configurations detailed, and shows that on either spacer material $J_{s c}$ tends to increase then level off with increasing thickness. This confirms that even if the spacer layers are increased further the absolute $J_{s c}$ in Table 4.2 are optimal. The levelling off of $J_{s c}$ points to the increased anti-reflection abilities of the ITO spacer layer perfectly balancing out the decreased forward scattering by the MNPs. On a $\mathrm{SiO}_{2}$ spacer the performance hits a maximum and then decreases with increasing spacer thickness, leading to the 


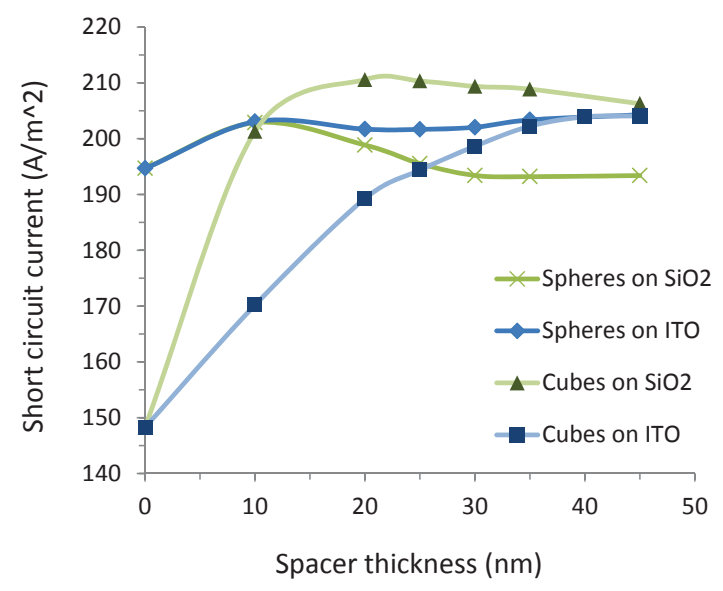

Figure 4.18: Best $J_{s c}$ for any surface coverage of $\mathrm{Al}$ nanocubes and nanospheres on increasingly thick ITO and $\mathrm{SIO}_{2}$ spacer layers.

observation that once the MNPs are raised too far they are no longer effective at forward scattering and the low index $\mathrm{SiO}_{2}$ layer does not perform well as an ARC.

Due to the almost ideal refractive index of ITO for ARC purposes we should expect the ITO spacer layers to give large short circuit currents despite their less than ideal properties for MNP forward scattering. Table 4.2 demonstrates that this is the case, and both spheres and cubes on ITO come within $3 \%$ of the best $J_{s c}$. The $\mathrm{SiO}_{2}$ spacer actually gives rise to the best overall short circuit currents despite being a poor ARC. This is due to the extremely favourable conditions that a low index material provides for MNP forward scattering.

Table 4.2 also confirms the results of our earlier discussions and optimizations demonstrating that $\mathrm{Al}$ nanocubes on a simple $\mathrm{SiO}_{2}$ or ITO spacer layer outperform a plain ARC by $6 \%$ and $2.6 \%$ respectively. The system and the $J_{s c}$ in Fig. 4.18 could be further improved upon by examining the behaviour of nanocubes inside an ITO layer or with a double ITO/SiO 2 coating to provide both good electrical contact and the low RI environment for optimal forward scattering. 


\subsubsection{Particle scattering and field enhancement}

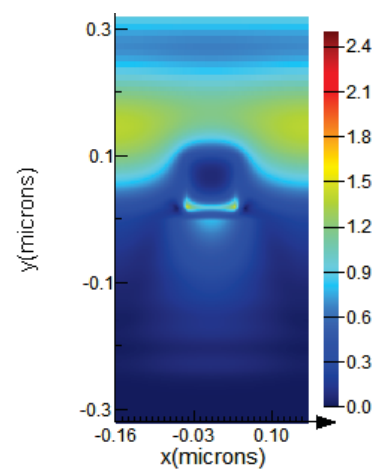

(a)

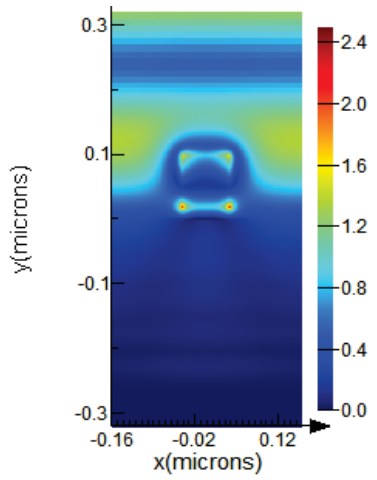

(b)

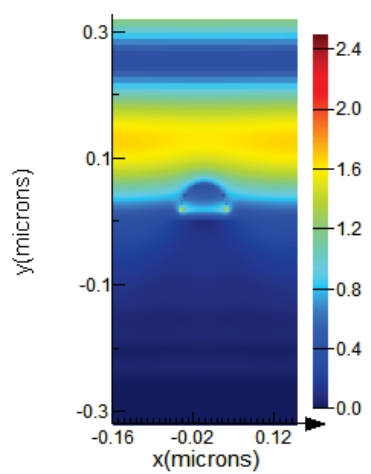

(c)

Figure 4.19: $|E|$ at the solar spectrum peak, $500 \mathrm{~nm}$, on a plane passing through the center of the particle parallel to the direction of polarization. Field intensity shown for 80 $\mathrm{nm}$ a) Al cubes, b) Ag cubes and c) Ag hemispheres on 20 $\mathrm{nm}$ ITO on a-Si.

Snapshots of the $|E|$ were taken for $\mathrm{Al}$ and $\mathrm{Ag}$ nanocubes on a $20 \mathrm{~nm}$ ITO/240 $\mathrm{nm}$ a-Si stack at $\lambda=500 \mathrm{~nm}$ to determine nanoparticle behaviour at the solar spectrum peak. These are shown in Fig. 4.19 along with the field around a Ag hemisphere in the same configuration. We can compare this behaviour to the observed generation rate in the silicon for one period of a solar cell structure. Fig. 4.20 describes the generation rate for solar cells with a 20nm ITO spacer layer and differing shapes and materials of MNPs. The generation rate is calculated over all relevant wavelengths in the solar spectrum. We can clearly see the effect of the MNPs on the generation rate, assuming that each absorbed photon contributes an $e^{-} / h^{+}$pair.

The short circuit currents derived from the $\mathrm{Al}$ cube, Ag cube, Ag hemisphere and bare cell configurations are $19.1 \mathrm{~mA} / \mathrm{cm}^{2}, 16.2 \mathrm{~mA} / \mathrm{cm}^{2}, 14.8 \mathrm{~mA} / \mathrm{cm}^{2}$ and $16.2 \mathrm{~mA} / \mathrm{cm}^{2}$ respectively. Where the two $\mathrm{Ag}$ MNPs tend to provide a reduction in generation rate directly underneath the particle at the a-Si surface, the Al MNP shows 


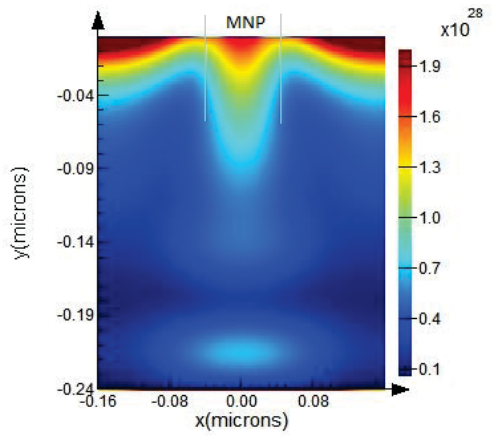

(a)

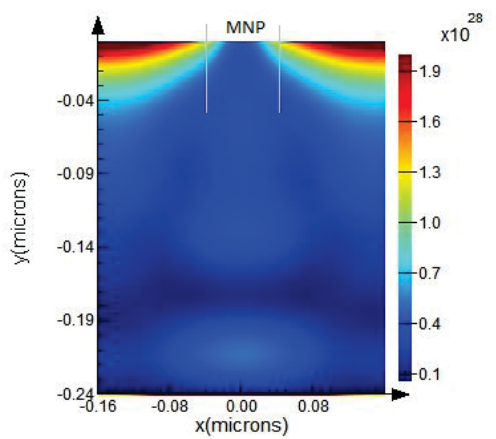

(c)

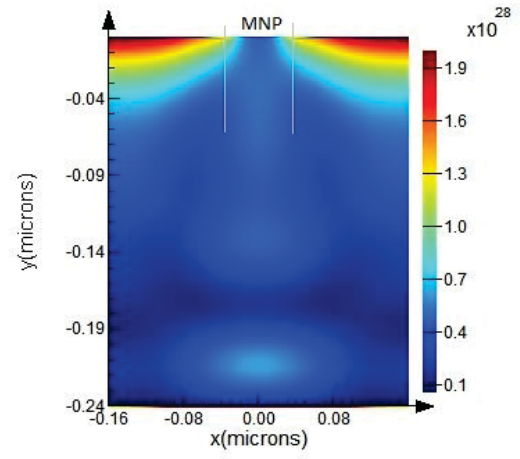

(b)

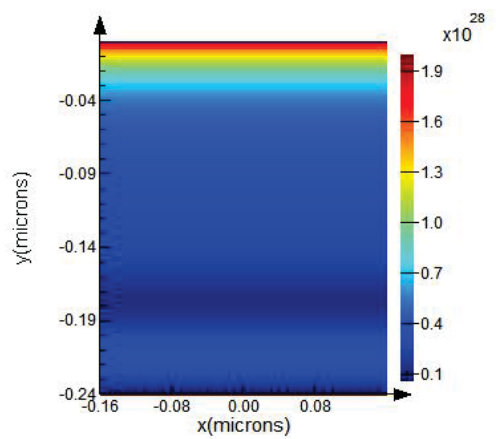

(d)

Figure 4.20: Generation rate (charge pairs $/ \mathrm{m}^{3} / \mathrm{s}$ ) in a-Si solar cells coated with $20 \mathrm{~nm}$ ITO and $25 \%$ surface coverages of $80 \mathrm{~nm}$ a) Al cubes, b) Ag cubes, c) Ag hemispheres and d) no MNPs. The lines show the approximate $x$-position of the MNPs. All colour scales are identical.

a large portion of light scattered forward into the silicon layer below, both directly below the particle and beside it. The reason Al cubes outperform their Ag peers is clear from Fig. 4.19a. The Al cubes strongly forward-scatter underneath the MNP whereas the Ag MNPs exhibit minimal field penetration into the a-Si at the solar spectrum peak.

Fig. 4.20a shows a high generation rate underneath the $\mathrm{Al}$ nanocubes while the Ag MNPs actually show lower generation rates underneath the particle than would be expected from a cell with no MNPs on top. Fig. 4.19 also shows that the $|E|$ is concentrated much more highly inside the Ag MNPs while there is almost no E field 


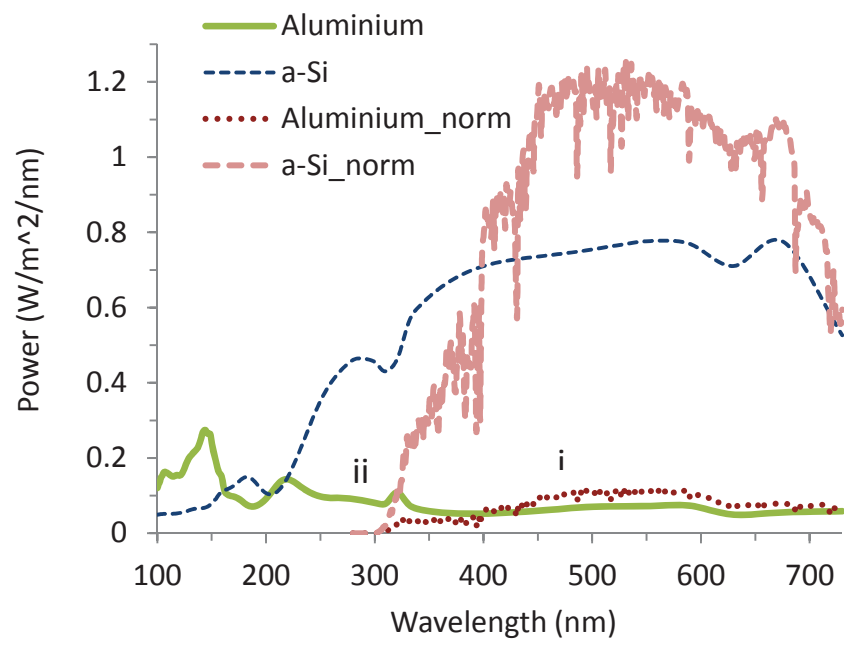

Figure 4.21: $80 \mathrm{~nm} \mathrm{Al}$ cube on 20nm ITO showing resonant modes as Al absorption peaks, mostly in the UV. The dipolar (i) and quadrupolar(ii) modes are shown at $483 \mathrm{~nm}$ and $315 \mathrm{~nm}$ respectively.

penetration into the Al MNP. This enables much lower absorption inside the Al MNPs as compared with the Ag MNPs. This phenomenon is known as skin depth, or the depth that the electric field will penetrate into the material at a given wavelength. At $500 \mathrm{~nm}$ the skin depth is only $13 \mathrm{~nm}$ for $\mathrm{Al}$ and at least $25 \mathrm{~nm}$ for $\mathrm{Ag}$ [37] representing more than $30 \%$ of the Ag MNP diameter.

The key to the low absorption in the $\mathrm{Al}$ particles is also due in part to the location of the dipolar, quadrupolar and higher order resonances. Since most of the resonant modes in $\mathrm{Al}$ cubes appear outside the solar spectrum, a simulation into the deep-UV region, down to $100 \mathrm{~nm}$, was performed in Fig. 4.21. Here the dipolar and quadrupolar resonant modes are labelled at $483 \mathrm{~nm}$ and $315 \mathrm{~nm}$ respectively, while the other modes are visible as $\mathrm{Al}$ absorption peaks at higher frequencies. To emphasize the fact that absorption in the $\mathrm{Al}$ nanocubes occurs mostly outside of the solar spectrum, the absorption values are normalized to the solar spectrum power at each wavelength, labelled Al cube_solar. No absorption occurs in the Al below 300nm, where the major 
$\mathrm{Al}$ absorption peaks are located, since no power is available from the sun below $300 \mathrm{~nm}$. Within the solar spectrum metal absorption stays below $0.15 \mathrm{~W} / \mathrm{m}^{2} / \mathrm{nm}$, even at the current $25 \%$ surface coverage of nanocubes.

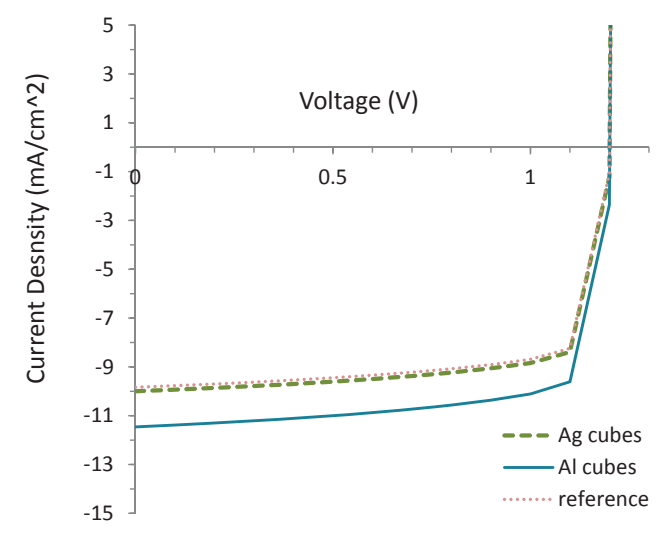

Figure 4.22: Simulated I-V curve for a) Al nanocubes and b) Ag nanocubes on a $20 \mathrm{~nm}$ ITO spacer layer. Efficiencies are $7.51 \%$ and $6.56 \%$ respectively, a cell with no MNPs is $6.45 \%$ efficient.

To further demonstrate the viability and validate the results of the proposed enhancement method, electrical simulations were also performed in Lumerical's DEVICE ${ }^{\circledR}$, taking into account material properties such as electron-hole mobility and recombination effects. The material parameters and script for calculating the I-V curve can be found in Appendix A.3. Simulations are performed using the a-Si properties of Table 3.1 and the generation rates determined in Fig. 4.20. The I-V curves of the enhanced and reference solar cells are calculated and shown in Fig. 4.22. The bare cell with only a $20 \mathrm{~nm}$ ITO layer was $6.45 \%$ efficient while the cell with $\mathrm{Al}$ nanocubes on top reached $7.51 \%$ efficiency compared to only $6.56 \%$ for $\mathrm{Ag}$ nanocubes. This represents a more than $1 \%$ increase in absolute cell efficiency over a bare cell or Ag nanocubes when using optimally situated Al nanocubes. The electrical simulations prove that $V_{o c}$ is not affected by addition of the MNPs, only the photo-generated current is affected. 


\section{Chapter 5}

\section{Experimental Demonstration}

\subsection{Fabrication Methods}

Originally, this study focused on amorphous silicon solar cells as it was thought they could be fabricated relatively easily at the Micro/Nano Fabrication Laboratory here at Carleton University. First generation test solar cells were fabricated in n-i-p substrate configuration using the structure of Fig. 5.1, with the a-Si layers deposited via PVD in a Varian sputter system. Doping was achieved by having pre-doped targets for the sputtering process. The deposition parameters used can be found in Table 5.1. The I-V curves obtained from the test cells indicated that the cells were behaving much more like resistors and less like semiconductor diodes, producing zero current at short circuit conditions. We postulate that this is due to very low doping concentrations in the p-type and n-type layers due to the initial boron and phosphorous doping concentrations of the sputter targets. The material quality from PVD systems for solar cell purposes is also known to be very poor in terms of electron mobility. Any $e^{-} / h^{+}$pairs that were generated were not collected efficiently due to low mobility and low carrier drift from the lack of any substantial built in electric field.

These challenges were overcome by using a nanocrystalline silicon (nc-Si) and 
Table 5.1: Parameters used for deposition of Si and ITO

\begin{tabular}{ccccc}
\hline Process & Temperature & Pressure & RF Power & Deposition Rate \\
\hline \hline a-Si (PVD) & $250^{\circ} \mathrm{C}$ & 5 mTorr & $200 W$ & $67 \AA / \mathrm{min}$ \\
nc-Si (PECVD) & $280^{\circ} \mathrm{C}$ & 900 mTorr & $2 W$ & $20 \AA / \mathrm{min}$ \\
ITO (PVD) & $100^{\circ} \mathrm{C}$ & $8 \mathrm{mTorr}$ & $1000 \mathrm{~W}$ & Scan speed $90 \mathrm{~cm} / \mathrm{min}$ \\
\hline
\end{tabular}

protocrystalline (pc-Si) thin-film in the same configuration as Fig. 5.1, in place of a-Si. nc-Si is composed of tiny regions of crystalline silicon in amongst a matrix of amorphous silicon, thus utilizing the excellent electrical properties of c-Si and the high absorption coefficient of a-Si. In addition, pc-Si is a silicon phase that occurs right at the transition between a-Si and c-Si. The obstacles currently facing commercial nc-Si are low deposition rates and high cost of fabrication; however, for purposes of the current experiment nc-Si should be an excellent candidate as a test platform.

We note here that the optical simulations performed in earlier sections still apply to the experiments at hand and should be an accurate indicator of performance as the refractive indices of a-Si and nc-Si are very similar [56] due to the fact that nc-Si is simply small crystalline portions of silicon embedded in a mostly amorphous material. In fact, nc-Si has a slightly lower refractive index than a-Si and based on our studies (Fig. 4.3) this should result in a higher overall $J_{s c}$ and larger enhancements from the nanoparticles due to an increase in forward scattering.

With this in mind, second generation solar cells were fabricated in collaboration with the Centre for Advanced Photovoltaic Devices and Systems (CAPDS) at the University of Waterloo. CAPDS deposited the nanocrystalline silicon (nc-Si) and protocrystalline silicon (pc-Si) in the substrate n-i-p configuration. Next, arrays of metal nanocubes were formed on the ITO defined active solar cell area via e-beam lithography at the University of Toronto using a Vistec EBPG 5000+ Electron Beam 
Lithography (EBL) System. The EBPG 5000+ is a state-of-the-art Gaussian beam vector scanning EBL system giving extremely accurate results as seen by the regular arrays in Fig. 5.5. Intermediate steps and any other processing was completed in the Micro/Nano Fabrication Laboratory at Carleton University.

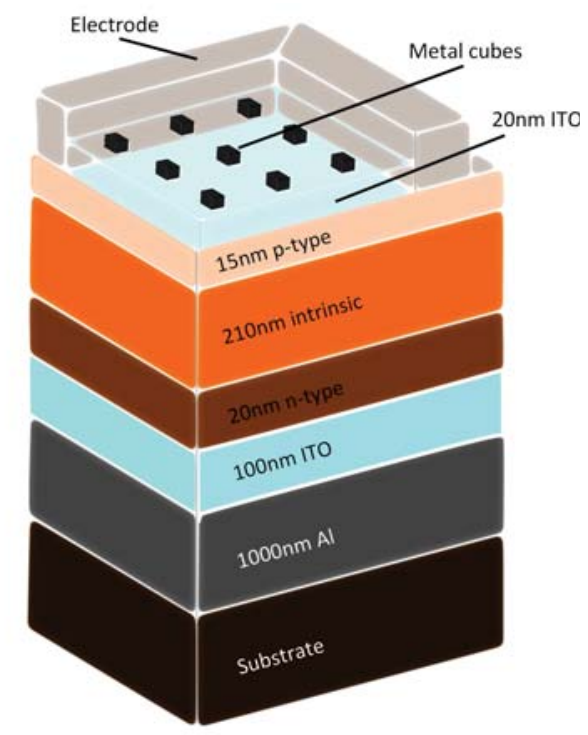

Figure 5.1: Thin-film nc-Si cell cut-out profile

Thin-film solar cell fabrication was completed following the depiction in Fig. 5.1. A 2" 100 n-type crystalline silicon wafer was used as a structural base. We then evaporated $1 \mu \mathrm{m}$ of $\mathrm{Al}$ as a back contact for the cells. Following that a $100 \mathrm{~nm}$ coating of ITO was sputtered on as a recombination barrier at the silicon-metal interface [57]. An n-i-p substrate configuration is now appropriate for this structure. $20 \mathrm{~nm}$ of n-type nc-Si was deposited by PECVD using the parameters of Table 5.1, followed by $210 \mathrm{~nm}$ of intrinsic pc-Si and a further $15 \mathrm{~nm}$ of p-type nc-Si. Next, an etch mask (Fig. 5.2) was applied by photolithography to define the back contacts for the cells. 


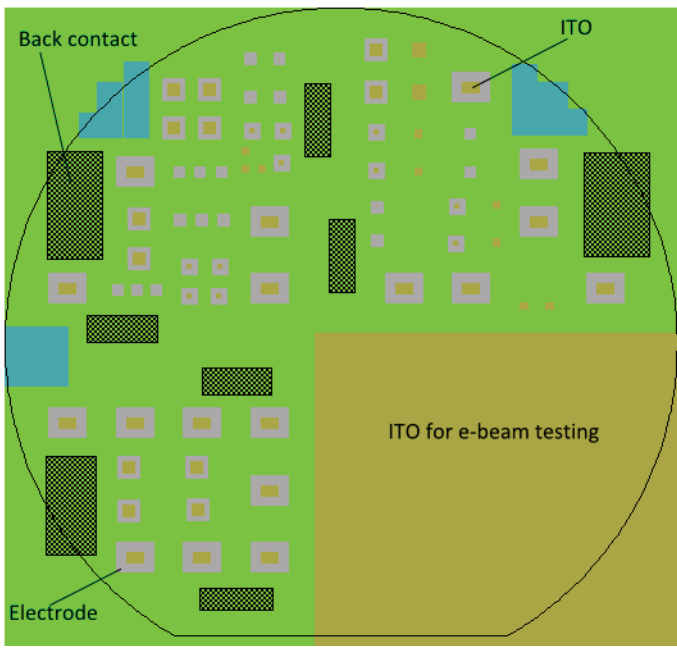

(a)

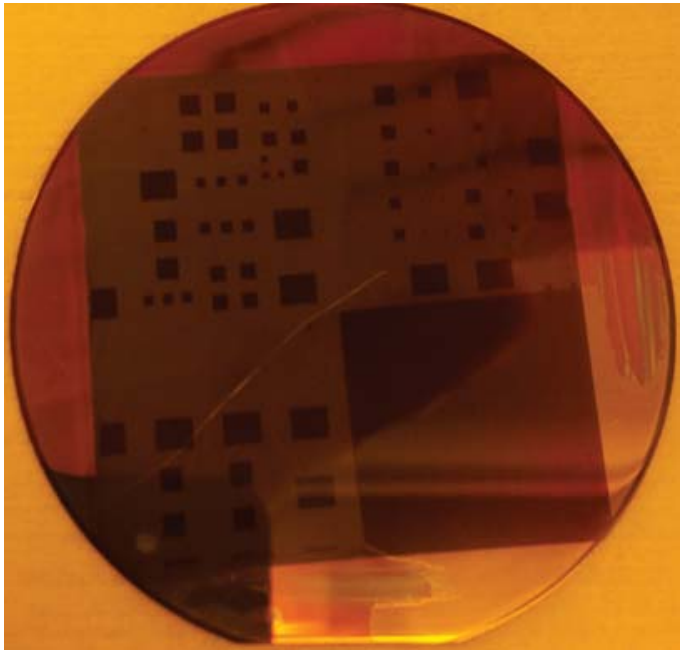

(b)

Figure 5.2: a) L-Edit mask for defining solar cell back Al contacts, active ITO area and top $\mathrm{Al}$ contacts and b) actual processed wafer with ITO top contacts defined.

Wafer masking procedure is as follows:

- $200^{\circ} \mathrm{C}$ hotplate bake for $10 \mathrm{~min}$

- Liquid HMDS spin coat

- Spin coat LOR $10 B$

- Hotplate bake at $190^{\circ} \mathrm{C}$ for 5 min

- Spin coat S1811 positive photoresist at 1000rpm (10s), 4000rpm (30s)

- Prebake at $125^{\circ} \mathrm{C}$ for $1 \mathrm{~min}$

- Mask alignment

- $59 s$ exposure under UV light

- Develop for $50 \mathrm{~s}$

The nc-Si was then etched through using Technic etch with Al acting as the etch-stop and then the remaining resist removed in Microposit Remover $1165^{\circledR}$.

A $20 \mathrm{~nm}$ ITO layer was then sputter coated in a using a Semicore sputter tool. After the ITO deposition the surface of the wafer appears as in Fig. 5.3 under 40kx 
magnification. The surface roughness varies from $172 \mathrm{~nm}$ to $620 \mathrm{~nm}$ after correction by a factor of $\sqrt{2}$ due to the $45^{\circ} \mathrm{C}$ inclination of the image sensor relative to the sample.



Figure 5.3: nc-Si surface with 20nm ITO deposited.

A second photolithographic mask was applied and the ITO subsequently etched to create $500 \mu m \times 500 \mu m, 200 \mu m \times 200 \mu m$ and $100 \mu m \times 100 \mu m$ squares which define the active solar cell areas. The ITO etch is carried out in Aqua regia, a solution composed of $40 \mathrm{~mL} \mathrm{HNO}_{3}$ added to $160 \mathrm{~mL}$ HCL and then $200 \mathrm{~mL} \mathrm{H} \mathrm{H}_{2} \mathrm{O}$ kept at $45^{\circ} \mathrm{C}$. A $20 \mathrm{~s}$ dip is sufficient to etch $20 \mathrm{~nm}$ of ITO. We assume that the relatively low lateral conductivity of nc-Si implies each individual cell is defined by the top square of ITO. E-beam lithography with metal lift-off was then used to create arrays of $80 \mathrm{~nm} \times 80 \mathrm{~nm} \times 80 \mathrm{~nm} \mathrm{Al} \mathrm{nanocubes}$ with pitches (array size) $380 \mathrm{~nm}(500 \mu \mathrm{m} \times 500 \mu \mathrm{m})$, $320 \mathrm{~nm}(500 \mu \mathrm{m} \times 500 \mu \mathrm{m}), 260 \mathrm{~nm}(200 \mu \mathrm{m} \times 200 \mu \mathrm{m})$ and $140 \mathrm{~nm}(100 \mu \mathrm{m} \times 100 \mu \mathrm{m})$. $180 \mathrm{~nm}$ thick PMMA was used as a photoresist for the e-beam lithography process. An array of square holes was defined by the electron beam in the resist. After developing 
the resist a Balzers electron beam evaporator was used to evaporate $80 \mathrm{~nm}$ of $\mathrm{Al}$ onto the samples. Finally the resist was lifted off by soaking overnight in acetone bath plus a 1 min ultrasonic clean. A further $5 \min$ in $80^{\circ} \mathrm{C}$ Microposit Remover $1165^{\circledR}$ ensured all resist was removed. This last removal step was found to have no negative impact on the nc-Si or ITO layers. Finally, a top Al electrode was applied via photolithography around the individual cells. The whole process is detailed pictorially in Appendix B.

The end result is depicted in Fig. 5.4 showing one of the $500 \mu m \times 500 \mu m$ array of nanocubes (outlined in grey) sitting on top of a larger $20 \mathrm{~nm}$ thick ITO spacer layer and surrounded by a 300 $\mathrm{nm}$ thick Ag electrode. The nanocube array is slightly off-center due to multiple arrays being written on the same sample not all of which were centred on their respective ITO areas. The offset is measured at $50 \mu \mathrm{m}$ both vertically and horizontally, only a $10 \%$ error in both directions. This should have minimal impact on the finished final results.

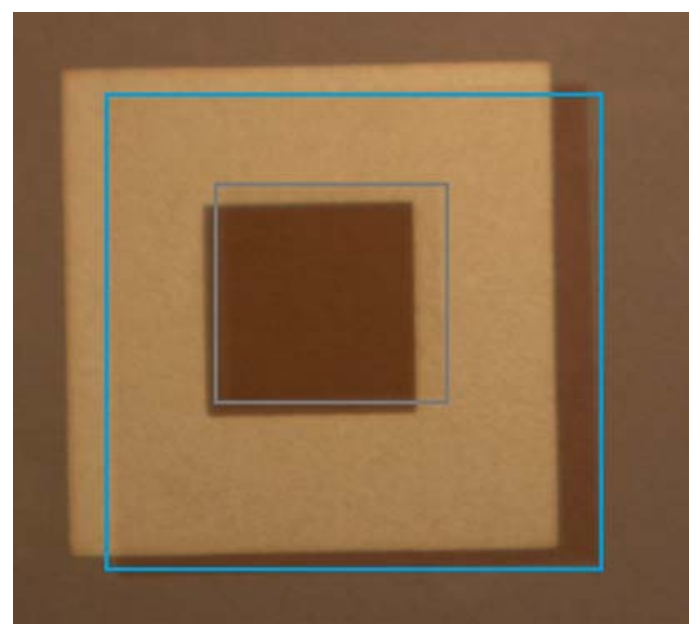

Figure 5.4: Fully processed $500 \mu m \times 500 \mu m$ array after Ag top electrode deposition. The grey box outlines the nanocube array and the larger blue box outlines the ITO active area.

A scanning electron microscope was used to examine the samples after the deposition of the nanocube arrays. Fig. 5.5 shows the sample with several different array 
pitches. The nanocubes are measured to be $80 \pm 3 \mathrm{~nm}$ with extremely regular spacing (see Fig. 5.5a) due to the accuracy of the Vistec e-beam writer. Note the very cubic shape of the Al nanoparticles as well, the edges are slightly rounded but these have distinct flat edges thus distinguishing them from cylindrical shaped nanoparticles.

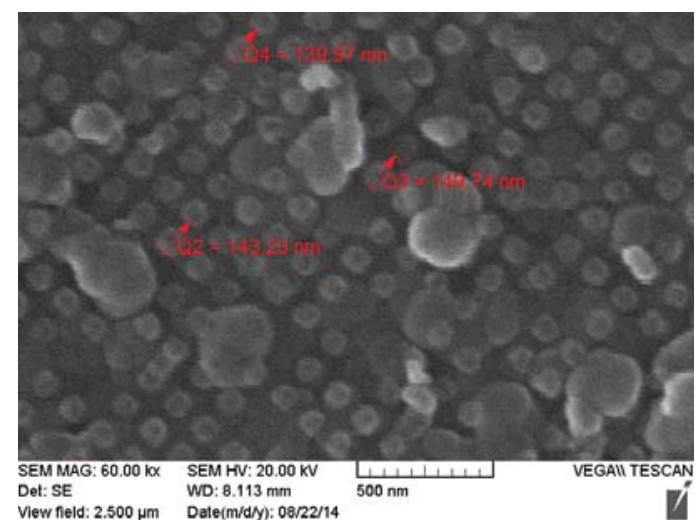

(a)

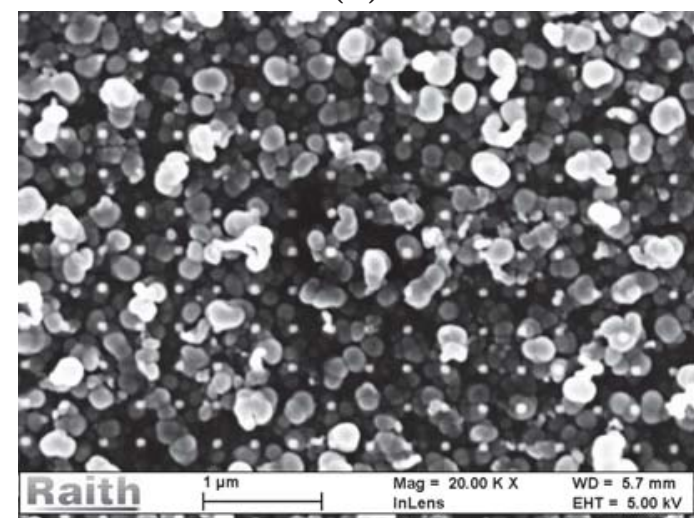

(c)

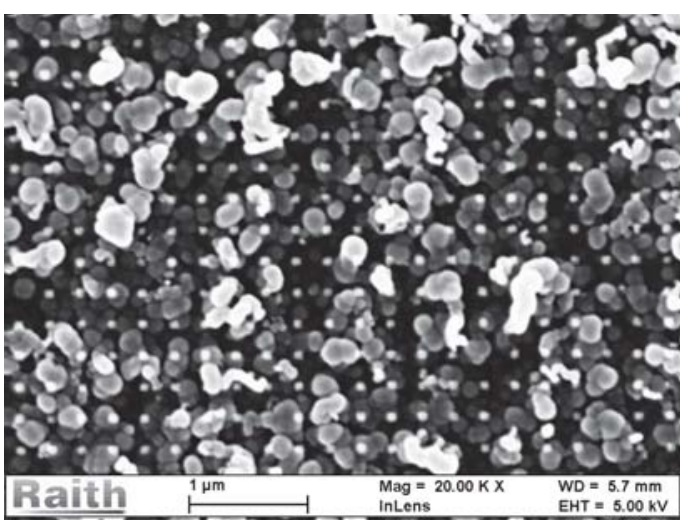

(b)

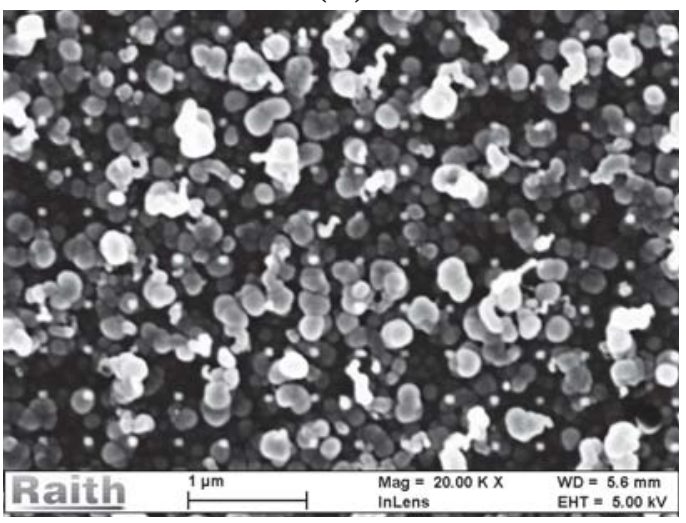

(d)

Figure 5.5: SEM images showing top down view of Al nanocube arrays with pitches a) $140 \mathrm{~nm}$, b) $260 \mathrm{~nm}$, c) $320 \mathrm{~nm}$ and d) $380 \mathrm{~nm}$. 


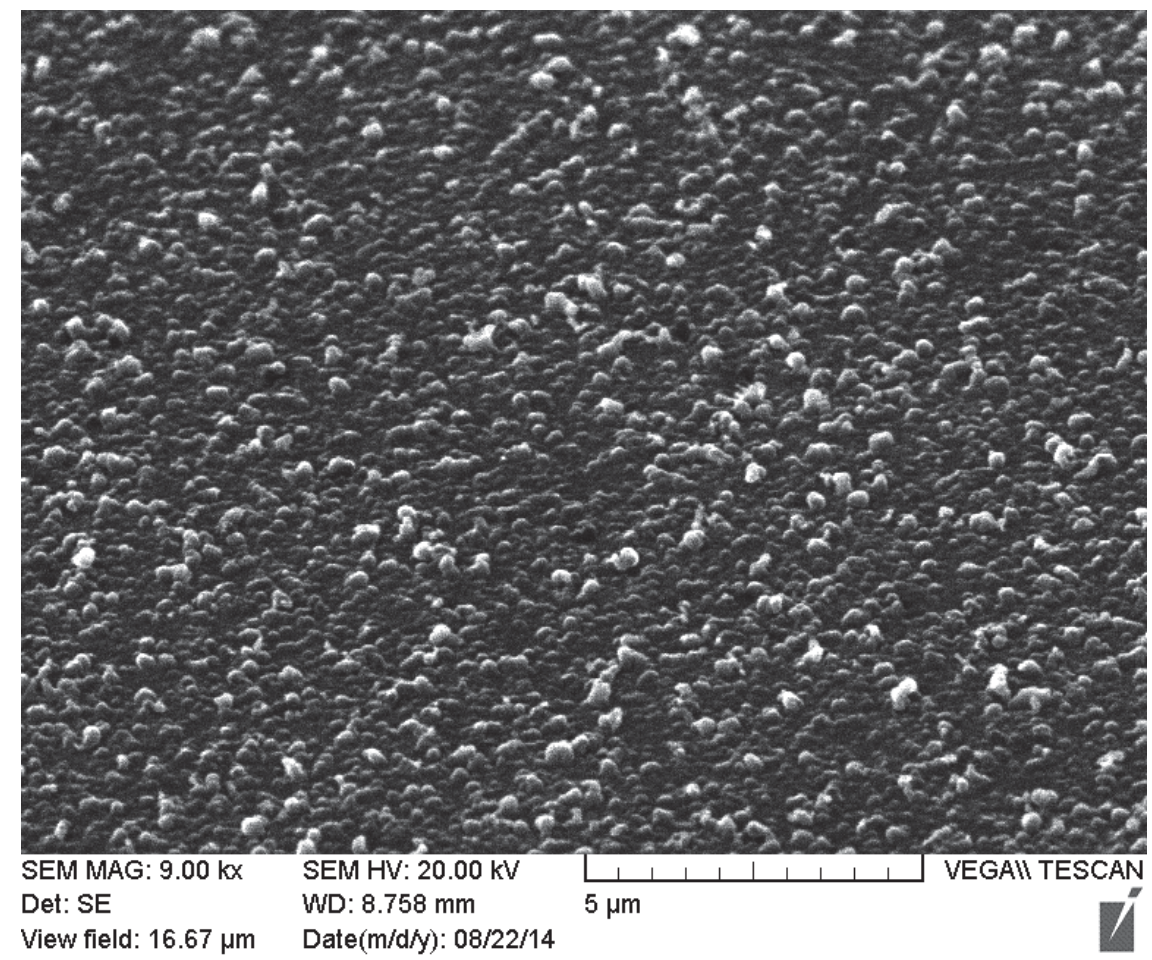

Figure 5.6: SEM image of nc-Si with $20 \mathrm{~nm}$ ITO taken at $9 \mathrm{k} \times$ magnification.

\section{$5.2 \quad$ Testing Results}

Arrays with $80 \mathrm{~nm}$ Al nanocubes were fabricated with pitches of $140 \mathrm{~nm}, 260 \mathrm{~nm}$, $320 \mathrm{~nm}$ and $380 \mathrm{~nm}$ corresponding to surface coverages of $33 \%, 9 \%, 6 \%$ and $4 \%$. We compare our results to that of Villesen et al. [29] who fabricated 100nm diameter $\mathrm{Al}$ discs on a $40 \mathrm{~nm} \mathrm{SiO}_{2}$ spacer with a $400 \mathrm{~nm}$ array pitch (5\% surface coverage) on a Si p-i-n photodiode and achieved a 3\% enhancement in photo-current. They stressed that this was only a proof-of-concept and so here we have attempted to build on that work.

Our simulations showed that the higher $33 \%$ surface coverages (140nm pitch) should provide the best increase in generated photo-current. However Hylton et al. also found, using numerical methods, that a smaller array pitch of $200 \mathrm{~nm}$ for 100nm diameter $\mathrm{Al}$ nanocylinders would provide the best enhancement. In that case, 


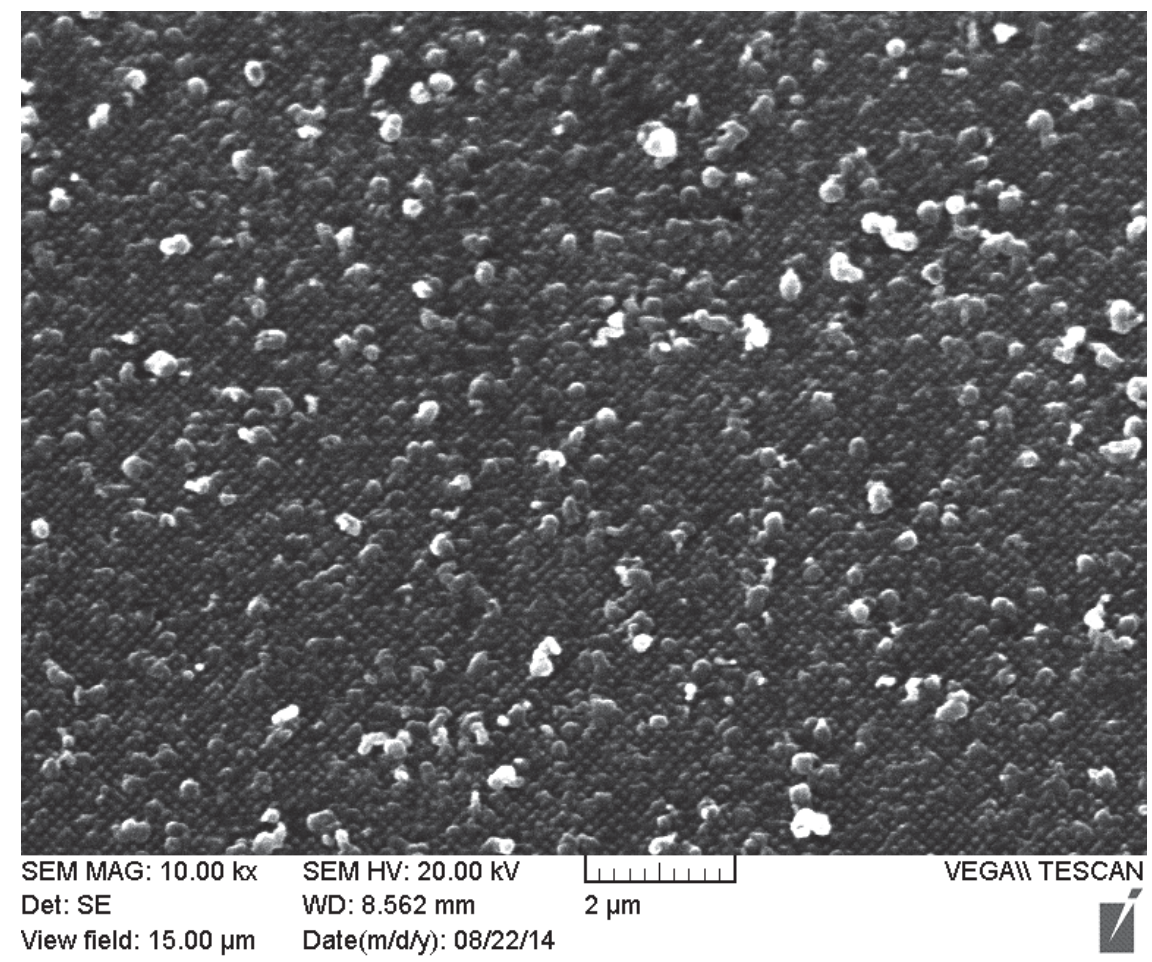

Figure 5.7: Zoomed out SEM image of $140 \mathrm{~nm}$ pitch array taken at $10 \mathrm{k} \times$ magnification. Nanocube array is clearly visible as faint grid structure.

experiments verified that the opposite was true and a $400 \mathrm{~nm}$ pitch provided much better results [30] though the exact reasons were indeterminate the variations from a perfect cylindrical shape was cited as a possible reason for the discrepancy. For this reason we have fabricated arrays with pitches spanning the range from $140 \mathrm{~nm}$ to $380 \mathrm{~nm}$.

After fabrication reflectance measurements were taken on an Oriel IQE 200 machine for cells with MNPs at a 380nm array pitch and identical cells without MNPs. In this case the cells have no top Ag electrode, only $1 \mathrm{~mm} \times 1 \mathrm{~mm}$ ITO pads, one of which has a $500 \mu m \times 500 \mu m$ array of nanocubes in the center. Simulations were also performed via FDTD and the results are compared in Fig. 5.8. The reference cell shows quite good agreement between simulation and experiment simply based on the shape of the curves and the location of the major features. Discrepancies arise from 
two major sources. First, the simulations use the a-Si refractive index which differs from that of the nc-Si in the experiments; however, the two are similar enough to provide insight into the behaviour of the reflectance.

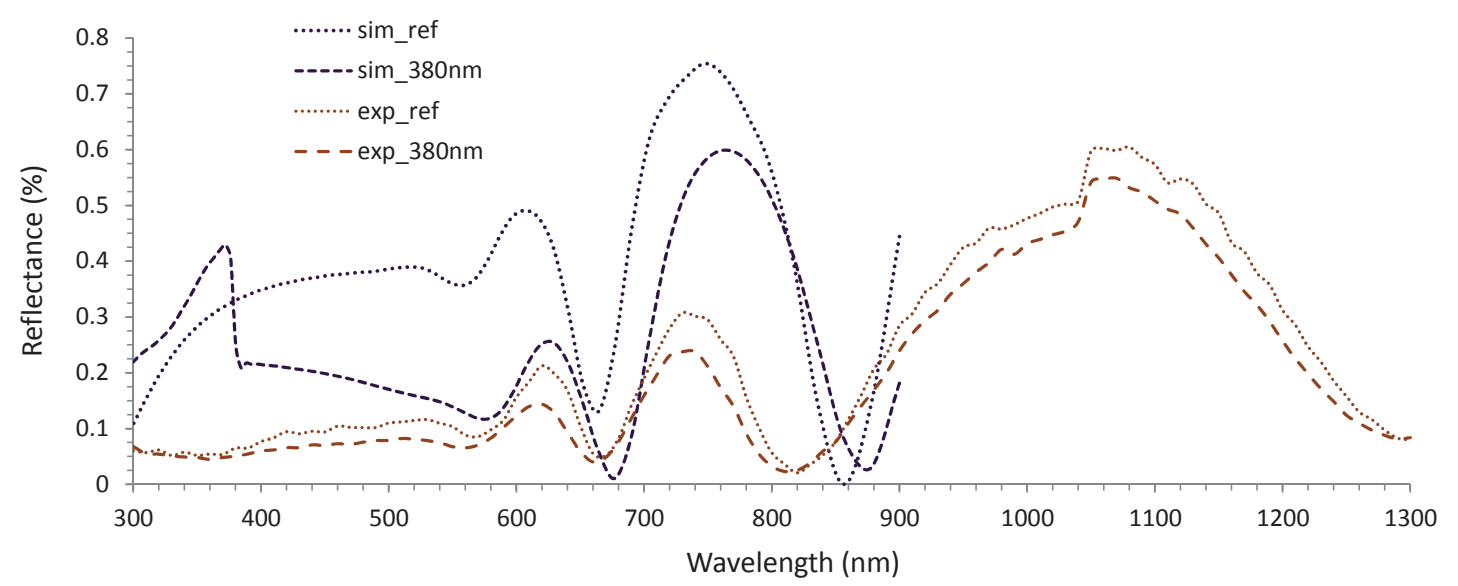

Figure 5.8: Reflectance simulations and measurements for a reference cell with $20 \mathrm{~nm}$ of ITO and a cell with Al nanocube array at $380 \mathrm{~nm}$ pitch.

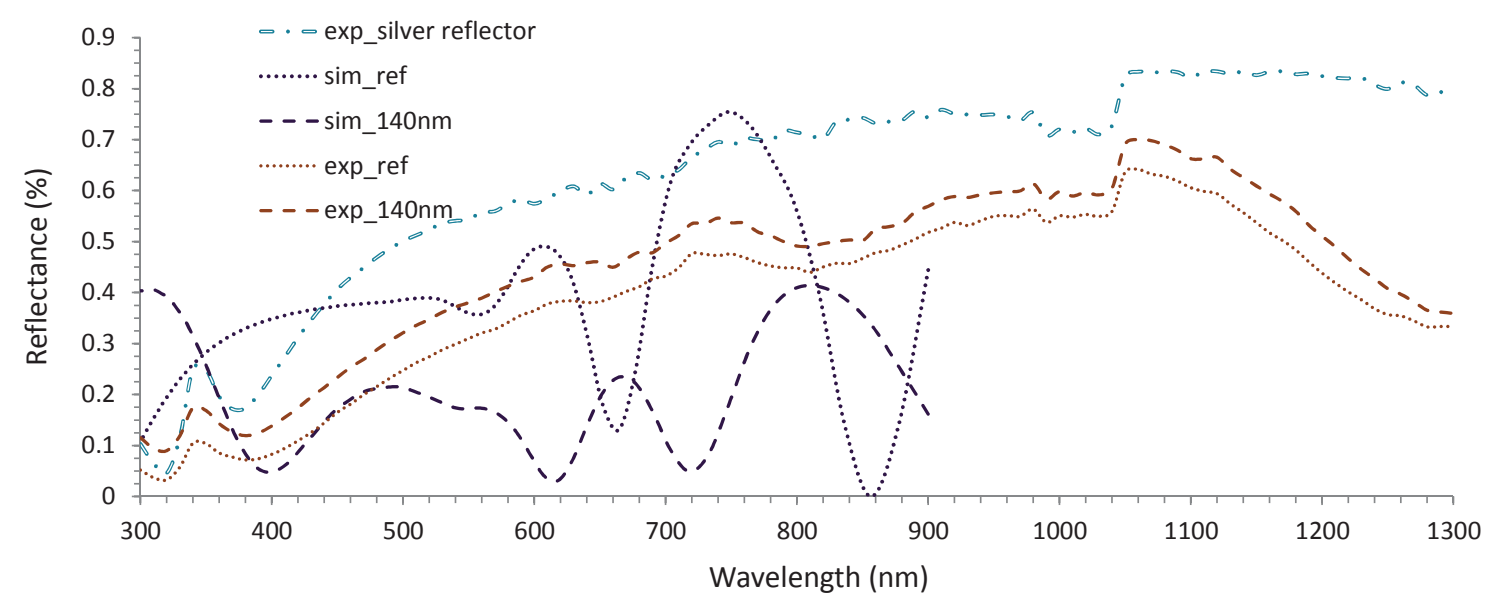

Figure 5.9: Reflectance simulations and measurements for a reference cell with $20 \mathrm{~nm}$ of ITO and a cell with Al nanocube array at $140 \mathrm{~nm}$ pitch. Also shown is the reflectance of $300 \mathrm{~nm}$ thick $\mathrm{Ag}$ surface on nc-Si.

The main reason the reflectance is so much lower in the experiments is found by examining Fig. 5.3. From this we see that the nc-Si surface is very rough with 
features ranging in height from $170 \mathrm{~nm}$ all the way to $620 \mathrm{~nm}$. Figs. 5.6 and $5.7 \mathrm{show}$ zoomed out views of the surface with and without MNPs respectively. The roughness acts as an excellent anti-reflection coating and light-trapping structure. Interestingly the roughness appears to be greater than the thickness of the deposited silicon and ITO layers in some areas $(240+20 \mathrm{~nm})$. This indicates that the deposition created some "towers" of material as these large entities are relatively few in number and show up as bright spots in Figs. 5.6 and 5.7. The smaller surface roughness features can be attributed to the roughness of the underlying silicon wafer or back contact $\mathrm{Al}$ evaporation as well as the nc-Si growth process. The roughness of the surface has a large impact on the results, significantly reducing reflection from expected values in Fig. 5.8 (sim_ref) to the measured values (exp_ref).

A second reflectance measurement is done for arrays of cubic nanoparticles which have a pitch of $140 \mathrm{~nm}$, or approximately a $33 \%$ surface coverage in Fig. 5.9. We cannot compare directly the reflectance values from Figs. 5.8 and 5.9 because the beam spot size of the measurement setup was a semicircle with an area of $392700 \mu m^{2}$ compared to active areas of $250000 \mu \mathrm{m}^{2}$ and $10000 \mu \mathrm{m}^{2}$ for the $380 \mathrm{~nm}$ and $140 \mathrm{~nm}$ pitches respectively. The edges of the arrays are covered by wide silver top contact pads which are very reflective, thus we have used reference cells with identical dimensions but without MNPs on the surface in order to gain a good comparison of the effect of the MNPs on reflectance. The reflectance of a bare 300 $\mathrm{nm}$ thick Ag surface is also shown. Quite obviously the reflectance of the Ag top contact dictates the shape of the reflectance curves as they deviate enormously from the simulated response which does not take into account top contact reflectance. As we move to larger array sizes such as the $500 \times 500 \mu m 320 \mathrm{~nm}$ pitch array (Fig. 5.10) the silicon response starts to match the simulations. 


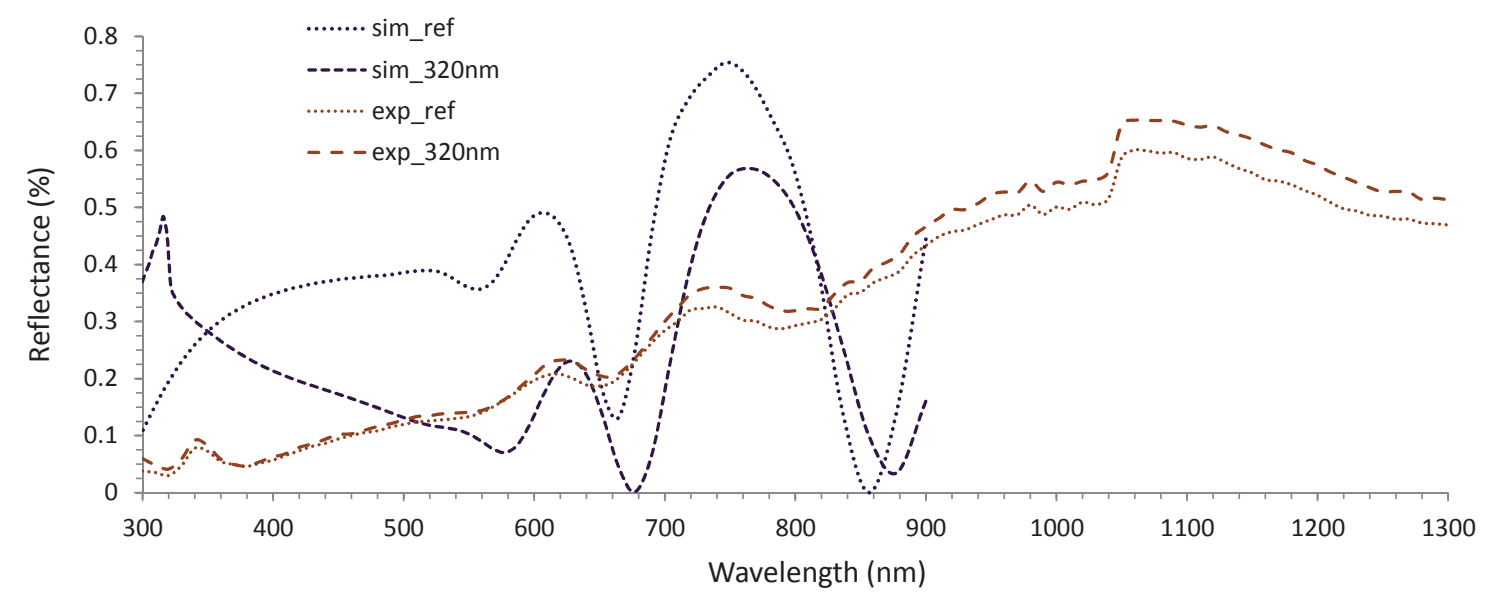

Figure 5.10: Reflectance simulations and measurements for a reference cell with $20 \mathrm{~nm}$ of ITO and a cell with Al nanocube array at $320 \mathrm{~nm}$ pitch.

Comparing the relative reflectance of each nanocube array to the reference cells it is clear that our experimental results are similar to those of Hylton et al. [30]. We can see that increasing the array pitch leads to less and less reflectance from the cells. The $140 \mathrm{~nm}$ pitch increases reflectance by $26.4 \%$, the $320 \mathrm{~nm}$ pitch increases reflectance by $7.45 \%$ and the $380 \mathrm{~nm}$ pitch reduces reflectance by $45 \%$ over the $300-730 \mathrm{~nm}$ wavelength range. This contradicts the simulation results and the reason for this is still under investigation.

A main difference between the $380 \mathrm{~nm}$ array pitch and the $320 \mathrm{~nm}$ array pitch (both $500 \mu m \times 500 \mu m$ ) is the lack of top contact around the latter and this may have an impact on the performance of the array even though the results are compared to identical reference cells. The rounded edges of the deposited nanocubes (Fig. 5.5a) could also play some part in this since ideal cubic nanoparticles were simulated and small variations in particle shape will give rise to important differences in plasmonic response [46].

A third major factor could be the polarization of light used in the simulations versus the measurements. In the simulations light is polarized parallel to the edge 
of the nanoparticles, whereas in the measurements the light is almost certainly not polarized parallel to an edge of the nanoparticle array. This effect could vary the results somewhat; however, based on 2D simulations and subsequent measurements in Fig. 5.8 for large pitch arrays the simulation trends should hold in the experimental results.

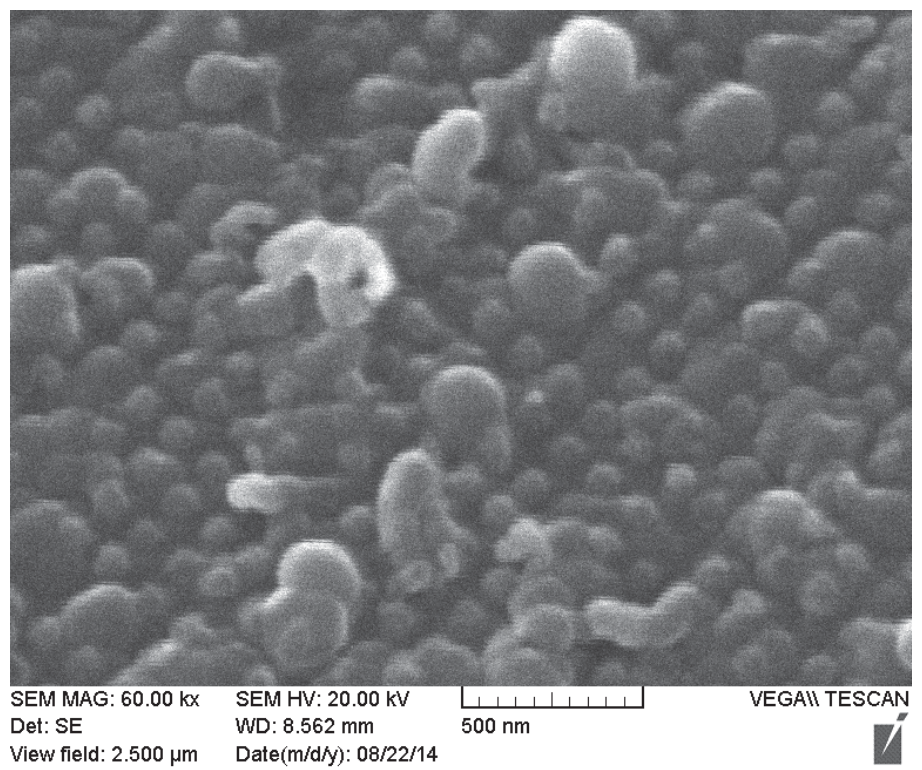

Figure 5.11: $80 \mathrm{~nm} \mathrm{Al}$ nanocube array with $140 \mathrm{~nm}$ pitch. Image taken at $25^{\circ}$ inclination and $60 \mathrm{k} \times$ magnification.

Clearly there is some effect as the arrays become more and more dense which is causing a variation from simulated values. One other possible reason in the current experiment is the surface roughness of the nc-Si. Fig. 5.11 shows a $25^{\circ}$ inclination view of the $140 \mathrm{~nm}$ pitch nanocube array. We can see that the MNPs are nestled in and amongst the larger silicon roughness features. Silicon beside the nanocubes will greatly affect their ability to scatter light in the forward direction as this high index material beside the MNPs will encourage light scattering into that material which is almost definitely p-type or coated in p-type material instead of forward into the rest of the substrate. Recall that most light absorbed in a doped amorphous 
silicon material is recombined very quickly due to the large number of defects. This improves slightly due to the nc-Si used here but still more than $50 \%$ of the material is amorphous and will recombine carriers very quickly. This would reduce the number of carriers available resulting in low voltage produced by the cell (see Fig. 5.13 below for further examination).

We also determined in Chapter 4 that a spacer layer of at least $20 \mathrm{~nm}$ was critical for forward scattering by nanocubes. It is possible that since the surface is so rough the 20nm of ITO deposited by PVD was not deposited evenly on the whole surface and may be missing in some areas. It is especially possible that the ITO is only covering the tops of the surface roughness features and did not settle into the areas in between. If this is the case the more tightly packed arrays would accentuate the problem leading to increased backscatter and an overall decrease in light trapping and photocurrent enhancement as detailed in Fig. 4.13a. If no spacer is present at high surface coverages we can expect to see decreases in cell performance $\left(J_{s c}\right)$ of up to $20 \%$ whereas for a low surface coverage (larger array pitch) no spacer layer would result in minimal decrease or minimal increase of up to $1 \%$ in $J_{s c}$.

The external quantum efficiency (EQE) was tested as well on an Oriel IQE 200 with the same semicircular beam having radius $1000 \mu m$. EQE was measured and then normalized to the solar spectrum and integrated over the $300-900 \mathrm{~nm}$ wavelength range.

Fig. 5.12 shows the experimentally measured EQE for solar cells with several differing $\mathrm{Al}$ nanocube array pitches on the top surface of $20 \mathrm{~nm}$ of ITO. The reason for the low EQE of all of the cells but the reference cell is the actual cell area was smaller than the EQE beam spot size with the surroundings being a Ag contact pad. Thus for all cells but the reference we can only measure relative EQE, the absolute value is most likely higher. The contact pad lead to large reflectivity of the smaller 
cells and hence a much lower EQE. We were unable to measure the EQE of identical reference cells in order to make a direct comparison between arrays. Thus we rely on the reflectance measurements to determine how the nanocubes are affecting the cell. Raw EQE measurements are shown for completeness in Fig. 5.12a, however the beam was non-uniform with an unknown profile. If we assume the beam follows a Gaussian distribution with $\sigma=0.15$ (i.e $f(x)=\exp \left(-x^{2} / 2 \sigma^{2}\right)$ and normalize the results to cell area based on this distribution we achieve Fig. 5.12b. These results follow roughly what we would expect from the reflectance measurements with the $380 \mathrm{~nm}$ array pitch being the only one to show enhancement over the reference cell. Looking at the $400-700 \mathrm{~nm}$ wavelength range the $380 \mathrm{~nm}$ pitch nanocube array is able to provide an increase of $7 \%$ in integrated EQE over the reference cell while the other two smaller array pitches showed decreases in performance. Combined with the reflectance measurements this confirms the results found by Hylton et al. [30] as noted earlier that the larger array pitch leads to a larger enhancement in contradiction with our simulation results.

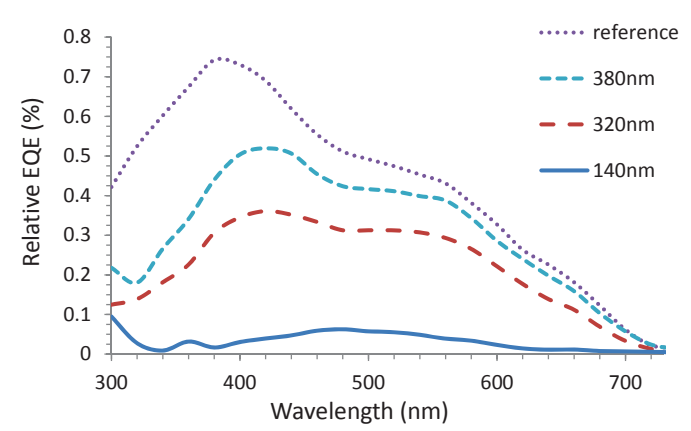

(a)

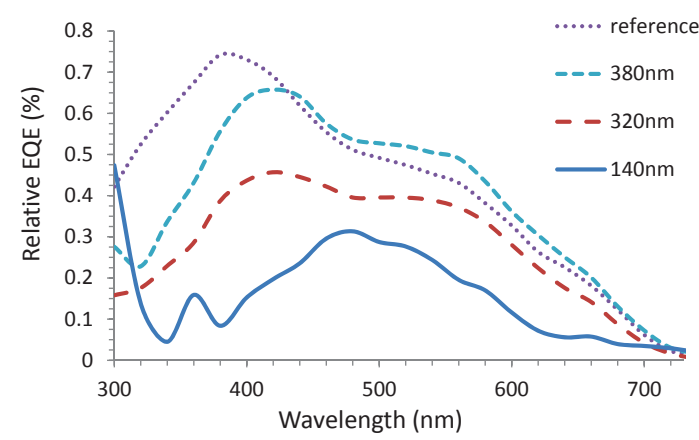

(b)

Figure 5.12: a) Raw EQE data for cells with varying array pitch b) Normalized EQE assuming beam is non-uniform with a Gaussian distribution $(\sigma=0.15)$

Cell performance was also evaluated under the AM1.5G solar spectrum at 1-sun concentration using an Oriel 92191 solar simulator incorporating a 1600W Xe lamp 
and $25^{\circ} \mathrm{C}$ cell temperature. This allowed measurement of the cell I-V curves and subsequently overall efficiency. Unfortunately we were unable to obtain an I-V curves for any cells with nanocube arrays due to extreme series and shunt resistances which prevented any sort of measurement. The resistances improved slightly on another sample which had several reference cells and an I-V curve was taken and analyzed below for a $1 \mathrm{~mm} \times 1 \mathrm{~mm}$ active area cell.

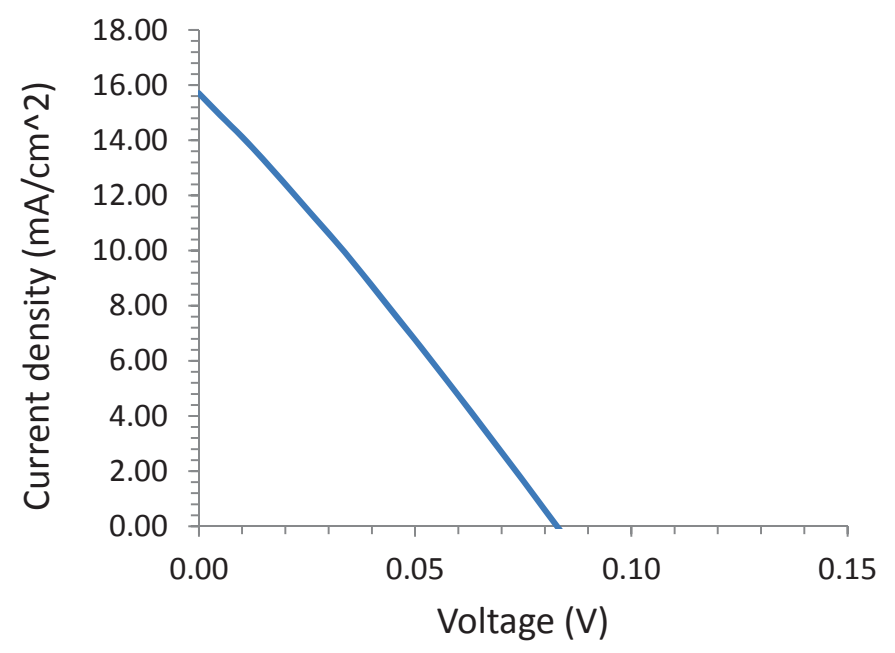

Figure 5.13: Experimental I-V curve for a $1 \mathrm{~mm} \times 1 \mathrm{~mm}$ defined active area nc-Si solar cell with no MNPs integrated.

As shown in Fig. 5.13 the solar cells as fabricated have an extremely low shunt resistance indicating that they have been shorted somewhere in the fabrication process. Shunt resistance normally occurs due to manufacturing defects and not poor cell design. We can calculate the shunt resistance approximately from the obtained I-V curve in Fig. 5.13 for a $1 \mathrm{~mm}^{2}$ cell with no MNPs. The shunt resistance is found from the slope of the curve near the short circuit point. Ideally shunt resistance is infinite, however for an almost ideal cell only values greater than $1000 \Omega \mathrm{cm}^{2}$ are required. On the other hand, a low shunt resistance drastically reduces the voltage produced by the cell since the current has a path alternative to the external circuit. We find here 
that the shunt resistance is approximately:

$$
\begin{aligned}
R_{s h} & =\frac{(0.06-0 \mathrm{~V})}{\left(15.7-4.8 \frac{\mathrm{mA}}{\mathrm{cm}^{2}}\right)} \times \frac{1000 \mathrm{~mA}}{1 \mathrm{~A}} \\
& =5.48 \Omega \mathrm{cm}^{2}
\end{aligned}
$$

The 20nm ITO layer could also play a part in reducing performance. At 100nm thickness, ITO exhibits a measured resistivity of $75 \Omega / \square$ when fabricated in a Semicore PVD. However, at 20nm ITO exhibits a $1000 \Omega / \square$ resistivity. In the current experiment with nc-Si as the active layer a TCO is not necessarily needed and thus this should not drastically affect the results. If an a-Si cell was used a much better or thicker TCO should be found.

Due to the use of nc-Si our original assertion of the active area only being defined by the ITO pads is not entirely correct. If this was true the $1 \mathrm{~mm}^{2}$ cell would have been producing $60 \mathrm{~mA} / \mathrm{cm}^{2}$, higher than the best reported c-Si solar cell. That assumption is correct based on a fully amorphous silicon material [16], however the nc-Si has better electrical properties and a 4-point probe measured sheet resistance of only $14 \Omega / s q$, which is similar to, or better than, a high quality crystalline silicon wafer. This means lateral conductivity of the nc-Si is much better than the a-Si so the cell will collect current from areas outside of the defined ITO region. If we instead assume that the active area collecting current is actually $2 m m \times 2 m m$ square we get the current densities of up to $16 \mathrm{~mA} / \mathrm{cm}^{2}$ depicted in Fig. 5.13 which are much closer to what would be expected for a nc-Si solar cell.

We can also calculate the sheet resistance based on the slope of the I-V curve near 
the open circuit voltage point.

$$
\begin{aligned}
R_{\text {ser }} & =\frac{(0.085-0.06 \mathrm{~V})}{\left.(2.11-(-0.2)) \frac{\mathrm{mA}}{\mathrm{cm}^{2}}\right)} \times \frac{1000 \mathrm{~mA}}{1 \mathrm{~A}} \\
& =4.80 \Omega \mathrm{cm}^{2}
\end{aligned}
$$

The poor $R_{\text {series }}$ and $R_{\text {shunt }}$ are the reason the I-V curve has a fill factor of only 0.27 under 1-sun illumination. Our test samples did not even allow a measurement above noise levels for the samples with nanocubes however the reflectance measurements are an excellent source of information regarding performance. If the nanoparticles are indeed sitting directly on the nc-Si substrate then our simulations match very well with the trends observed experimentally and the proposed enhancement method should be able to produce the desired $20 \%$ enhancement on ITO and $40 \%$ enhancement on $\mathrm{SiO}_{2}$. 


\section{Chapter 6}

\section{Conclusions}

This thesis covers the detailed design, simulation and optimization of arrays of plasmonic metal nanoparticles (MNPs) on the surface of thin film silicon solar cells. The MNPs exhibit resonant behaviour known as localized surface plasmon resonances. This resonant behaviour is exploited for improved light trapping and photocurrent generation in the active absorber layer of the thin-film solar cells without introducing extra surface recombination. We ultimately show that $80 \mathrm{~nm} \times 80 \mathrm{~nm} \times 80 \mathrm{~nm}$ aluminium (Al) nanocubes in periodic arrays on a thin indium-doped tin oxide (ITO), or ideally $\mathrm{SiO}_{2}$, spacer layer are optimal in terms of particle shape, size and metal choice for photocurrent enhancement in thin-film amorphous silicon (a-Si) solar cells. Additional benefits of $\mathrm{Al}$ include the ability to form $\mathrm{Al}_{2} \mathrm{O}_{3}$ meaning that oxidation occurs for only a few $n m$ before forming a stable compound. The cubic shaped nanoparticle also lends itself to accurate fabrication methods such as electron-beam lithography as opposed to the usually studied hemisphere and spherical particles which are extremely difficult to fabricate consistently, especially at high surface coverages.

The use of $\mathrm{Al}$ nanoparticles has only recently been examined in any detail for application to light trapping in solar cells via plasmonic MNPs. The use of Al represents a departure from previous works in that most have made use of silver (Ag) 
nanoparticles which have surface plasmon resonances in the VIS-near IR range. Al has resonances in the UV region which allows detrimental parasitic absorption to be shifted outside of the peak solar spectrum range, ensuring that enhancement rather than suppression of the photocurrent response occurs over the entire solar spectrum at energies above the band-gap for a-Si, $1.7 \mathrm{eV}$. Al nanocubes enable broadband photocurrent enhancement which is a major step towards viable use of plasmonic MNPs for light trapping in commercial thin-film solar cell applications.

The finite-difference time-domain method was used to perform all optical 3D simulations of the MNP properties. Once the behaviour of all the different shape/material combinations was determined the simulations were switched to $2 \mathrm{D}$ for analysis of a physical thin-film solar cell geometry. From this platform extensive optimizations were performed to determine the best materials and configurations for photocurrent enhancement. Increases in short circuit current $\left(J_{s c}\right)$ of up to $39 \%$ were shown for $80 \mathrm{~nm} \mathrm{Al}$ nanocubes on a $25 \mathrm{~nm} \mathrm{SiO}_{2}$ spacer layer compared with just $28 \%$ for hemispherical Al particles. Spherical MNPs were also examined and showed a $37 \%$ increase in $J_{s c}$, however the conditions for reaching that level of enhancement are not conducive to repeatable fabrication. In particular perfectly spherical particles cannot be accurately formed with lithographic methods and colloidal deposition or self-assembly methods are unsuitable for achieving regular particle spacing, especially at small array pitches (high surface coverage).

Investigating nanoparticle performance further on an ITO spacer layer (required to compensate for low lateral conductivity of a-Si), we discovered that $\mathrm{Al}$ nanocubes enhanced $J_{s c}$ by $20.4 \%$ at $35 \%$ surface coverage on a $20 \mathrm{~nm}$ ITO layer. This is in contrast to $\mathrm{Ag}$ nanocubes which only provide a 5.8\% increase and Ag hemispheres which actually reduce solar cell performance.

Finally, fabrication of a thin-film solar cell was carried out with arrays of $\mathrm{Al}$ 
nanocubes on the top surface to verify the simulation results. A 240nm nanocrystalline (nc-Si) active layer was used for the experiments as it was difficult to find an a-Si fabrication facility. After deposition of a $20 \mathrm{~nm}$ ITO layer and then the nanocube arrays it was discovered that the reference cells performed very poorly and efficiency measurements were unobtainable for most cells due to a small $R_{\text {shunt }}$ and large $R_{\text {series }}$. Using instead reflectance measurements it was determined that the nanocube arrays actually increased the reflectance of the device and decreased EQE for all except the $380 \mathrm{~nm}$ array pitch. It was postulated that this decrease was due to the surface roughness of the nc-Si and subsequent lack of ITO on the surface where nanocubes were formed. If the nanoparticles are indeed sitting directly on the nc-Si substrate then our simulations match very well with the trends observed experimentally. The proposed enhancement method should be able to produce the desired $20 \%$ enhancement on ITO and $40 \%$ enhancement on $\mathrm{SiO}_{2}$ with better fabrication and usage of a smoother a-Si active layer instead of nc-Si.

This thesis focused on optimizing and improving thin-film a-Si solar cells by increasing light trapping and photocurrent generation. We determined via simulations that using Al nanoparticles, with plasmon resonances outside of the solar spectrum, enabled broadband increases in light absorption in a-Si cells. In particular we utilized a cubic particle geometry which has not been looked at in the literature. We showed that the novel Al nanocube combination unlocked a $20.4 \%$ increase in short circuit current on a thin ITO spacer layer and if the refractive index of the spacer layer is lowered to that of $\mathrm{SiO}_{2}$ the inclusion of an array of $\mathrm{Al}$ nanocubes at $25 \%$ surface coverage on a $20 \mathrm{~nm}$ spacer layer improves short circuit current by $40 \%$. The proposed enhancement method uses inexpensive materials and straightforward fabrication techniques to provide large increases in short circuit current in thin film a-Si solar cells while not degrading open circuit voltage or increasing recombination in 
the cells. We have shown that surface plasmons are indeed a feasible method of light trapping in thin-film solar cell architectures.

\subsection{Future Work}

We demonstrated in this thesis the significant performance gains possible using $\mathrm{Al}$ nanocubes as plasmonic scatterers for light trapping in thin-film amorphous and nanocrystalline solar cells. Further simulations must be done to examine the effect of the shape factor, as described in Bohren and Huffman [40]. This is essentially the particle height to width ratio (aspect ratio) and further tuning could provide even better results. This is evident based on the large differences in enhancement factors reported by Villesen et al. and Hylton et al. for $\mathrm{Al}$ nanocylinders with aspect ratios of 1 and 0.5 respectively. Higher aspect ratio particles would lend themselves to nanoimprint lithography plus RIE etching and would be an natural extension to the current work. Further experimental verification should be carried out and a low-cost, high-throughput nanoimprint lithography mask could also be developed [58] in order to test the viability of the proposed light trapping scheme on commercially sized wafers.

The parameter space in which MNPs can be optimized for light trapping is highly complex. Further work must also be done to fully understand how MNPs operate in conjunction with a traditional anti-reflection coating for maximum absolute $J_{s c}$. The concept has been demonstrated, but not optimized, on commercial multicrystalline solar cells with small enhancements of $0.4 \mathrm{~mA} / \mathrm{cm}^{2}$ found using a sparse array of $100 \mathrm{~nm}$ nanospheres on a $75 \mathrm{~nm}$ silicon nitride AR coating [31]. We found that enhancements to cell performance were possible using nanocubes inside an ITO AR 
coating, though we did not elaborate on it. The results of Fig. 4.18 show that increasing the spacer thickness does not result in a continued increase in absolute $J_{s c}$. The combined system has trade-offs as well which must be balanced against one another and can contribute to thinner ITO coatings which will reduce costs as ITO is an expensive material. As the spacer layer (acting as an ARC) increases in thickness its anti-reflective properties increase; however, lifting the MNP off the substrate past the optimal point hinders it's ability to forward scatter. ARCs are better at reducing reflection than MNPs, however as we discovered the MNPs are superior in delivering large angle scattering and path length enhancement necessary for high performance thin-film solar cells. This could be a source of possible future optimizations and placement of the MNPs within the ARC would open up a whole new space for optimizations. This could easily be implemented on the current experimental platform and would be an interesting way to build off of the current work.

A continuation of the experimental work should be carried out with an a-Si active layer in order to obtain a smoother surface on which to deposit nanoparticles. Work needs to be done to determine if a $20 \mathrm{~nm}$ ITO layer is indeed feasible and if not the nanoparticles should be examined inside of a much thicker ITO layer in order to provide the requisite ITO resistivity as well as a spacer layer for optimal plasmonic performance of the nanocubes. The current work focuses on proving that Al nanoparticles, in particular nanocubes, are an excellent means of fabricating high performance thin-film solar cells. In that spirit further physical experiments are necessary using the spacer layer thickness detailed in Table 4.2 to determine if these do in fact provide superior performing cells in terms of best efficiency. Continuing to optimize the system in terms of the parameters described above could well lead to a commercially feasible process for the light absorption enhancement of thin-film solar cells. 


\subsection{Achievements and Publications}

- Veenkamp, R. J and Ye, W. N, Plasmonic metal nanocubes for broadband light absorption enhancement in thin-film a-Si solar cells, in Journal of Applied Physics, 115, 124317 (2014). (Impact factor: 2.21)

- Ryan J. Veenkamp, Shuyu Y. Ding, Ian J. Smith, Winnie N. Ye, Silicon solar cell enhancement by plasmonic silver nanocubes, in Physics, Simulation, and Photonic Engineering of Photovoltaic Devices III, Photonics West, Proceedings of SPIE Vol. 8981 (2014).

- Veenkamp, R. J and Ye, W. N, Efficiency enhancement of a-Si solar cells by nonresonant plasmonic aluminium nanocubes, IEEE-Nano (2014).

- R. Veenkamp and W. Ye, ITO Spacer for Optimized Plasmonic Enhancement of Aluminium Nanocubes on a-Si Solar Cells, Poster Presentation at IEEE Group IV Photonics (2014).

- Oral presentation at Photonics North, Investigation of aluminium and silver nanocubes for plasmonic enhancement of amorphous silicon solar cells (2014).

- Elected Vice-president, SPIE Carleton University Student Chapter.

- Awarded SPIE Officer Travel Grant to attend the SPIE Student Leadership Workshop at Photonics Europe. 


\section{List of References}

[1] J. Nelson, The Physics of Solar Cells. Series on Properties of Semiconductor Materials, Imperial College Press, 2003.

[2] J. Tsao, N. Lewis, and G. Crabtree, "Solar FAQs," US Dep. Energy, pp. 1-24, 2006.

[3] UNDP/WEC, "World Energy Assessment," tech. rep., New York, NY, 2000.

[4] D. Feldman, G. Barbose, and R. Margolis, "Photovoltaic (PV) Pricing Trends: Historical, Recent, and Near-Term Projections," No. November, (Golden, CO), NREL, U.S Department of Energy, 2012.

[5] J. Szlufcik and S. Sivoththaman, "Low-cost industrial technologies of crystalline silicon solar cells," Proc. IEEE, vol. 85, no. 5, pp. 711-730, 1997.

[6] D. Mulvaney, "Solar's green dilemma," Spectrum, IEEE, 2014.

[7] L. Ji and V. V. Varadan, "Competing effects of field localization and absorption enhancement using plasmonic nanostructures in thin film a-Si solar cells," in 2011 37th IEEE Photovolt. Spec. Conf., pp. 003353-003356, IEEE, June 2011.

[8] B. V. Aken, "Roll to roll fabrication process of thin film silicon solar cells on steel foil," in Photovolt. Spec. Conf., no. September, pp. 2-5, 2009.

[9] X. Deng and E. Schiff, "Amorphous silicon based solar cells," in Handb. Photovolt. Sci. Eng. (A. Luque and S. Hegedus, eds.), ch. 12, pp. 505-565, Chinchester: John Wiley \& Sons, 2003.

[10] W. Wang, L. Wang, and F. Liu, "Grain boundary passivation with small polar molecules for photovoltaics," in Photovolt. Spec. Conf., pp. 1486-1489, 2011.

[11] S. Pillai, K. R. Catchpole, T. Trupke, and M. A. Green, "Surface plasmon enhanced silicon solar cells," J. Appl. Phys., vol. 101, p. 093105, May 2007. 
[12] H. Stuart and D. Hall, "Island size effects in nanoparticle-enhanced photodetectors," Appl. Phys. Lett., vol. 73, no. 26, p. 3815, 1998.

[13] V. E. Ferry, M. A. Verschuuren, H. B. T. Li, E. Verhagen, R. J. Walters, R. E. I. Schropp, H. A. Atwater, and A. Polman, "Plasmonic light trapping for thin film A-SI:H solar cells," in 2010 35th IEEE Photovolt. Spec. Conf., pp. 000760000765, IEEE, June 2010.

[14] K. Kelly and E. Coronado, "The optical properties of metal nanoparticles: the influence of size, shape, and dielectric environment," J. Phys. Chem. B, vol. 107, pp. 668-677, 2003.

[15] K. R. Catchpole, S. Pillai, and K. L. Lin, "Novel applications for surface plasmons in photovoltaics," in 3rd World Conf. Photovolt. energy Convers., (Osaka), 2003.

[16] D. Derkacs, S. H. Lim, P. Matheu, W. Mar, and E. T. Yu, "Improved performance of amorphous silicon solar cells via scattering from surface plasmon polaritons in nearby metallic nanoparticles," Appl. Phys. Lett., vol. 89, p. 093103, Aug. 2006.

[17] K. Nakayama, K. Tanabe, and H. A. Atwater, "Plasmonic nanoparticle enhanced light absorption in GaAs solar cells," Appl. Phys. Lett., vol. 93, p. 121904, Sept. 2008.

[18] S. H. Lim, W. Mar, P. Matheu, D. Derkacs, and E. T. Yu, "Photocurrent spectroscopy of optical absorption enhancement in silicon photodiodes via scattering from surface plasmon polaritons in gold nanoparticles," J. Appl. Phys., vol. 101, no. 10, p. 104309, 2007.

[19] C. Hagglund, M. Zach, G. Petersson, and B. Kasemo, "Electromagnetic coupling of light into a silicon solar cell by nanodisk plasmons," Appl. Phys. Lett., vol. 92, p. 053110, Feb. 2008.

[20] K. R. Catchpole and A. Polman, "Design principles for particle plasmon enhanced solar cells," Appl. Phys. Lett., vol. 93, no. 19, p. 191113, 2008.

[21] F. J. Beck, A. Polman, and K. R. Catchpole, "Tunable light trapping for solar cells using localized surface plasmons," J. Appl. Phys., vol. 105, p. 114310, June 2009.

[22] H. A. Atwater and A. Polman, "Plasmonics for improved photovoltaic devices.," Nat. Mater., vol. 9, pp. 205-13, Mar. 2010. 
[23] D. M. Schaadt, B. Feng, and E. T. Yu, "Enhanced semiconductor optical absorption via surface plasmon excitation in metal nanoparticles," Appl. Phys. Lett., vol. 86, no. 6, p. 063106, 2005.

[24] T. Temple, G. Mahanama, H. Reehal, and D. Bagnall, "Influence of localized surface plasmon excitation in silver nanoparticles on the performance of silicon solar cells," Sol. Energy Mater. Sol. Cells, vol. 93, pp. 1978-1985, Nov. 2009.

[25] Y. Liu and J. Kim, "Absorption enhancement in thin film photovoltaic devices based on surface plasmon-polariton," in 2009 34th IEEE Photovolt. Spec. Conf., pp. 001471-001474, IEEE, June 2009.

[26] J. B. Lassiter, H. Sobhani, J. a. Fan, J. Kundu, F. Capasso, P. Nordlander, and N. J. Halas, "Fano resonances in plasmonic nanoclusters: geometrical and chemical tunability.," Nano Lett., vol. 10, pp. 3184-9, Aug. 2010.

[27] Z. Ouyang, S. Pillai, F. Beck, O. Kunz, S. Varlamov, K. R. Catchpole, P. Campbell, and M. A. Green, "Effective light trapping in polycrystalline silicon thin-film solar cells by means of rear localized surface plasmons," Appl. Phys. Lett., vol. 96, no. 26, p. 261109, 2010.

[28] F. J. Beck, S. Mokkapati, A. Polman, and K. R. Catchpole, "Asymmetry in photocurrent enhancement by plasmonic nanoparticle arrays located on the front or on the rear of solar cells," Appl. Phys. Lett., vol. 96, p. 033113, Jan. 2010.

[29] T. F. Villesen, C. Uhrenfeldt, B. Johansen, J. L. Hansen, H. U. Ulriksen, and A. N. Larsen, "Aluminum nanoparticles for plasmon-improved coupling of light into silicon.," Nanotechnology, vol. 23, p. 085202, Mar. 2012.

[30] N. P. Hylton, X. F. Li, V. Giannini, K.-H. Lee, N. J. Ekins-Daukes, J. Loo, D. Vercruysse, P. Van Dorpe, H. Sodabanlu, M. Sugiyama, and S. A. Maier, "Loss mitigation in plasmonic solar cells: aluminium nanoparticles for broadband photocurrent enhancements in GaAs photodiodes.," Sci. Rep., vol. 3, p. 2874 , Jan. 2013.

[31] Y. Zhang, X. Chen, Z. Ouyang, H. Lu, B. Jia, Z. Shi, and M. Gu, "Improved multicrystalline Si solar cells by light trapping from Al nanoparticle enhanced antireflection coating," Opt. Mater. Express, vol. 3, p. 489, Mar. 2013. 
[32] Y. Akimov, W. Koh, and K. Ostrikov, "Enhancement of optical absorption in thin-film solar cells through the excitation of higher-order nanoparticle plasmon modes," Opt. Express, vol. 17, p. 10195, June 2009.

[33] Y. A. Akimov and W. S. Koh, "Resonant and nonresonant plasmonic nanoparticle enhancement for thin-film silicon solar cells.," Nanotechnology, vol. 21, p. 235201, June 2010.

[34] Y. A. Akimov and W. S. Koh, "Design of Plasmonic Nanoparticles for Efficient Subwavelength Light Trapping in Thin-Film Solar Cells," Plasmonics, vol. 6, pp. 155-161, Oct. 2010.

[35] R. Brendel and H. Queisser, "On the thickness dependence of open circuit voltages of pn junction solar cells," Sol. energy Mater. Sol. cells, vol. 29, pp. 397-401, 1993.

[36] C. Wronski, "Amorphous silicon photovoltaics: order from disorder," Photovolt. Spec. Conf., pp. 1-6, 2000.

[37] M. Quinten, Optical Properties of Nanoparticle Systems. Weinheim, Germany: Wiley-VCH Verlag GmbH \& Co. KGaA, Jan. 2011.

[38] S. Zhang, K. Bao, N. J. Halas, H. Xu, and P. Nordlander, "Substrate-induced Fano resonances of a plasmonic nanocube: a route to increased-sensitivity localized surface plasmon resonance sensors revealed.," Nano Lett., vol. 11, pp. 165763, Apr. 2011.

[39] W. Warrick, R. Kappera, and M. Tayahi, "Enhanced optical absorption in thin film solar cells by surface plasmons," in Int. Conf. Photonics 2010, pp. 1-5, IEEE, July 2010.

[40] C. F. Bohren and D. R. Huffman, Absorption and Scattering of Light by Small Particles. Wiley Science Series, Wiley, 2008.

[41] N. Grigorchuk, "Radiative damping of surface plasmon resonance in spheroidal metallic nanoparticle embedded in a dielectric medium," JOSA B, vol. 29, p. 3404, Nov. 2012.

[42] A. W. Powell, M. B. Wincott, A. A. R. Watt, H. E. Assender, and J. M. Smith, "Controlling the optical scattering of plasmonic nanoparticles using a thin dielectric layer," J. Appl. Phys., vol. 113, no. 18, p. 184311, 2013. 
[43] M. A. Garcia, "Surface plasmons in metallic nanoparticles: fundamentals and applications," J. Phys. D. Appl. Phys., vol. 44, p. 283001, July 2011.

[44] SOPRA, "RefractiveIndex.INFO - Refractive index database," 2014.

[45] R. Krahne, G. Morello, A. Figuerola, C. George, S. Deka, and L. Manna, "Physical properties of elongated inorganic nanoparticles," Phys. Rep., vol. 501, pp. 75221, Apr. 2011.

[46] S. A. Maier, Plasmonics: Fundamentals and Applications. Springer, 2010.

[47] Lumerical, "Overcoming the Multi-wavelength FDTD Challenge — Photonic Design, Analysis, Optimization Solutions," 2014.

[48] Y. A. Akimov, W. S. Koh, S. Y. Sian, and S. Ren, "Nanoparticle-enhanced thin film solar cells: Metallic or dielectric nanoparticles?," Appl. Phys. Lett., vol. 96, no. 7, p. 073111, 2010.

[49] J. B. Seung and S. L. Koeng, "High-efficiency pin-type Amorphous Si Solar Cells Fabricated with a Low-electron-affinity Buffer Layer on the Front Electrode," J. Korean Phys. Soc., vol. 59, p. 443, Aug. 2011.

[50] S. Pillai, F. J. Beck, K. R. Catchpole, Z. Ouyang, and M. A. Green, "The effect of dielectric spacer thickness on surface plasmon enhanced solar cells for front and rear side depositions," J. Appl. Phys., vol. 109, no. 7, p. 073105, 2011.

[51] A. Centeno, J. Breeze, B. Ahmed, H. Reehal, and N. Alford, "Scattering of light into silicon by spherical and hemispherical silver nanoparticles," Opt. Lett., vol. 35, p. 76, Dec. 2010.

[52] Y. Zhang, Z. Ouyang, N. Stokes, B. Jia, Z. Shi, and M. Gu, "Low cost and high performance Al nanoparticles for broadband light trapping in Si wafer solar cells," Appl. Phys. Lett., vol. 100, no. 15, p. 151101, 2012.

[53] F.-J. Tsai, J.-Y. Wang, J.-J. Huang, Y.-W. Kiang, and C. C. Yang, "Absorption enhancement of an amorphous Si solar cell through surface plasmon-induced scattering with metal nanoparticles.," Opt. Express, vol. 18 Suppl 2, pp. A20720, June 2010.

[54] J. Jung and T. G. Pedersen, "Polarizability of supported metal nanoparticles: Mehler-Fock approach," J. Appl. Phys., vol. 112, no. 6, p. 064312, 2012. 
[55] T. F. Villesen, C. Uhrenfeldt, B. Johansen, and A. Nylandsted Larsen, "Selfassembled Al nanoparticles on $\mathrm{Si}$ and fused silica, and their application for $\mathrm{Si}$ solar cells.," Nanotechnology, vol. 24, p. 275606, 2013.

[56] H. Lee, S. Lee, H. Lee, and S. Choi, "Dielectric function of nano-crystalline silicon: Effect of hydrogen," J. Korean Phys. Soc., vol. 39, no. December, pp. 30$34,2001$.

[57] M. I. Kabir, S. a. Shahahmadi, V. Lim, S. Zaidi, K. Sopian, and N. Amin, "Amorphous Silicon Single-Junction Thin-Film Solar Cell Exceeding 10\% Efficiency by Design Optimization," Int. J. Photoenergy, vol. 2012, pp. 1-7, 2012.

[58] L. Guo, "Nanoimprint Lithography: Methods and Material Requirements," Adv. Mater., vol. 19, pp. 495-513, Feb. 2007. 


\section{Appendix A}

\section{Lumerical Post-processing Scripts}

\section{A.1 Scattering and Absorption Cross-sections (FDTD)}

This script processes the 3D simulations done in Chapter 4 in order to determine the Mie scattering and absorption efficiencies of various MNPs. The script determines the optical cross-section of Chapter $3, \sigma$, and divides by the geometric cross-section of the particular MNP in order to disciver the scattering and absorption efficiencies $Q_{\text {scat }}$ and $Q_{a b s}$ as in Equation 3.21. The script then writes all data to a text file for further plotting and analysis.

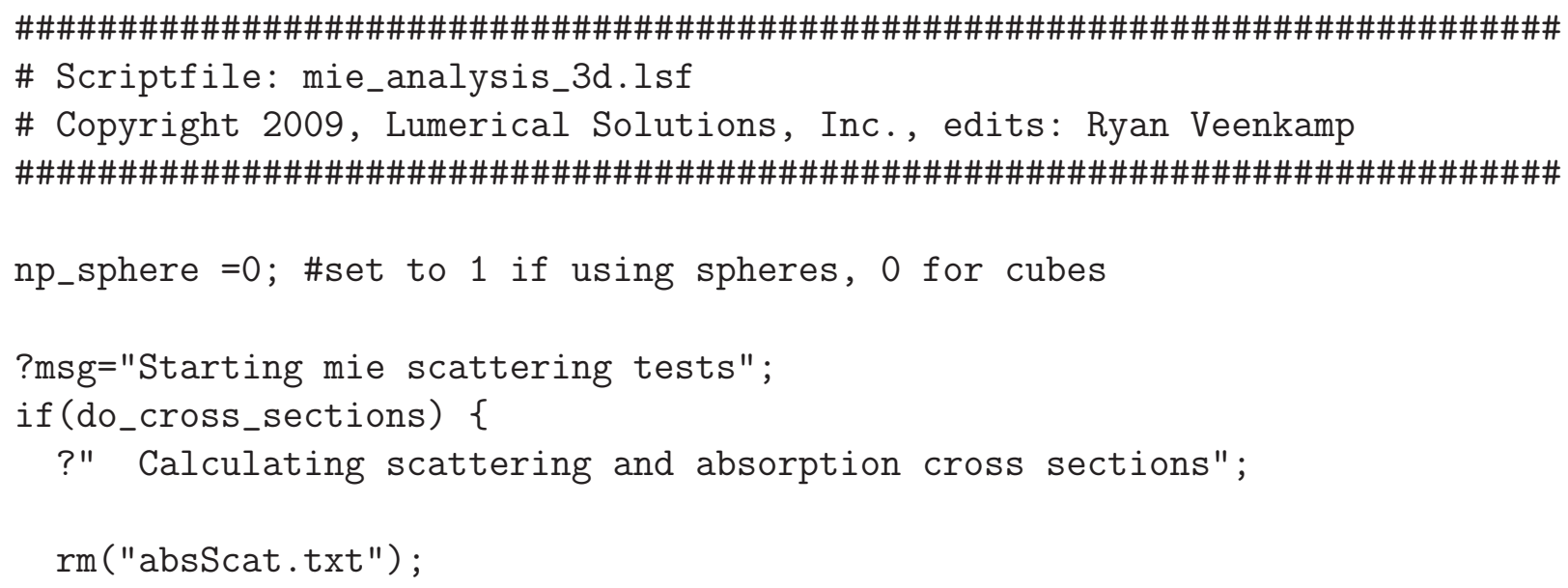




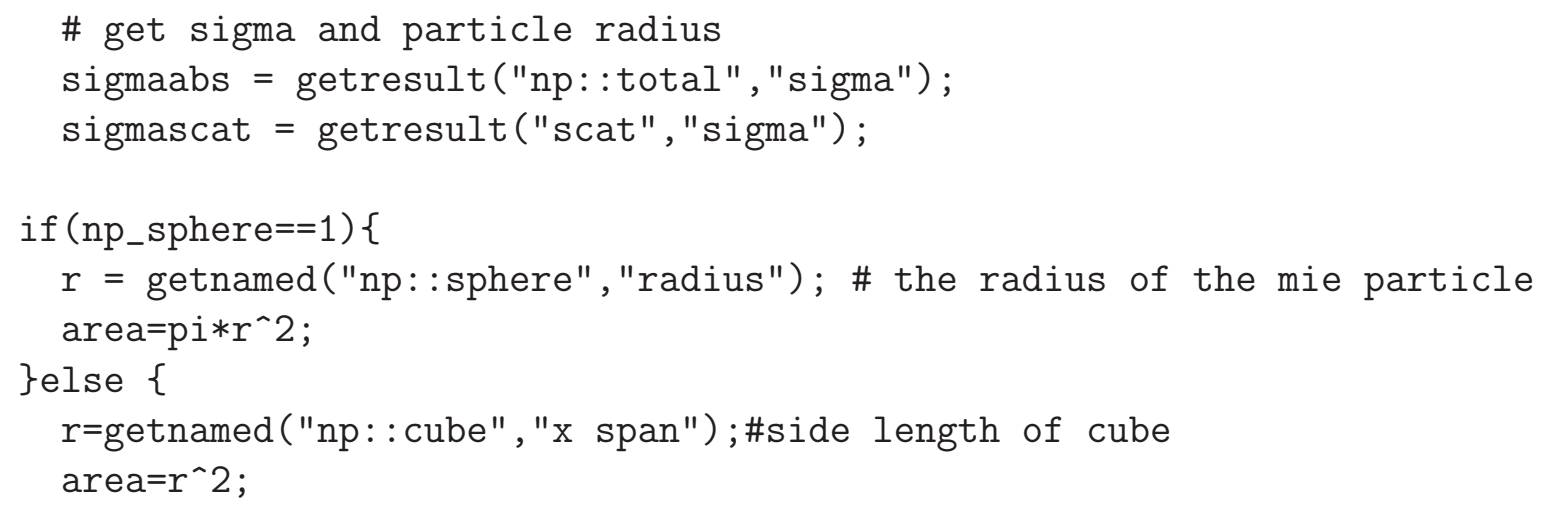




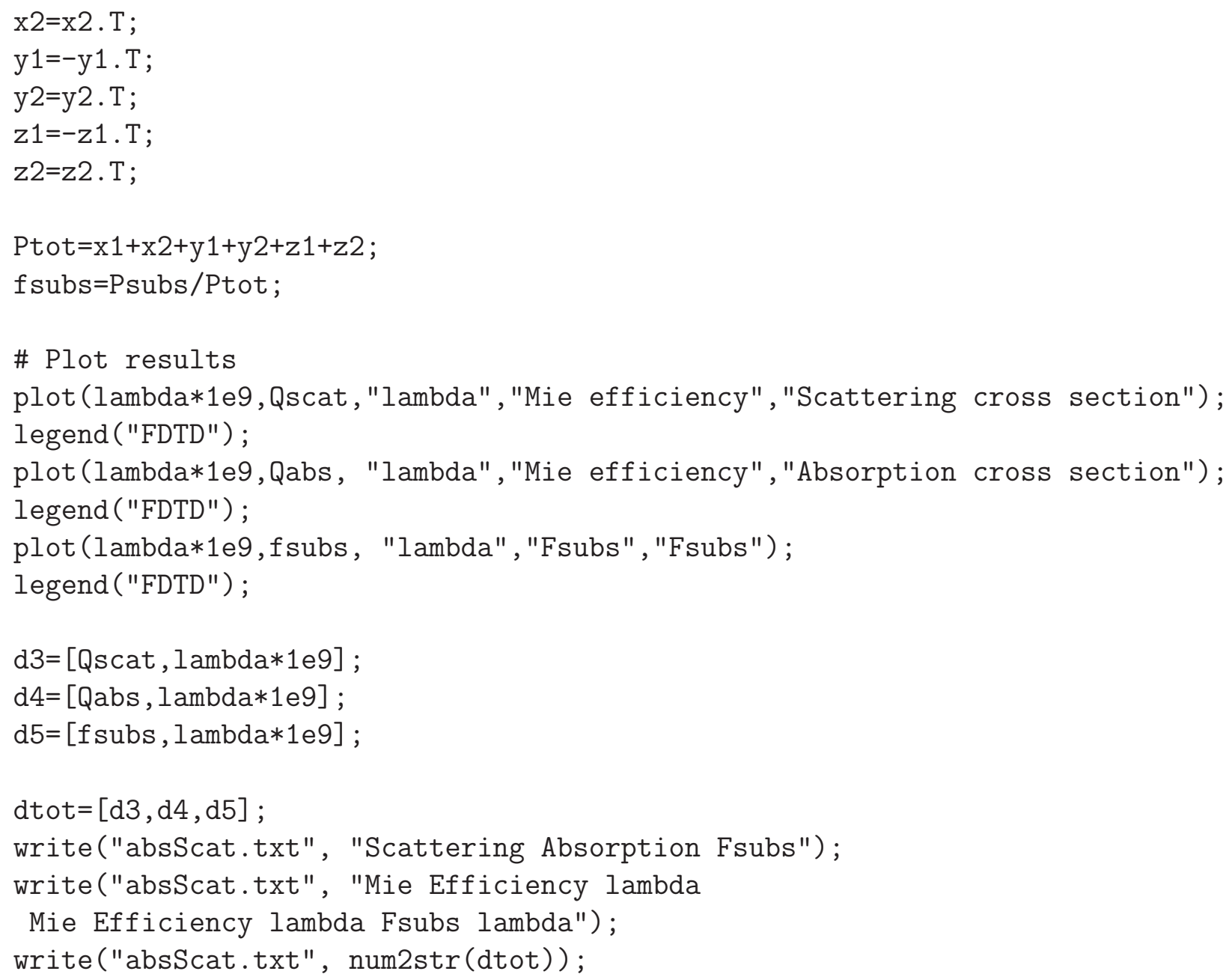




\section{A.2 Data analysis using solar spectrum data (FDTD)}

The following script is used to determine the power absorbed in the active silicon layer of a 2D solar cell as well as the power absorbed in the MNPs. The script calculates the absorption rate and then is able to interpolate the data to match the frequency vector of the AM1.5G solar spectrum. The absorption rate is then normalized to the power available in the solar spectrum at each wavelength to discover the total power absorbed in $W / \mathrm{m}^{2} / \mathrm{nm}$. Data is written to a text file for further processing.

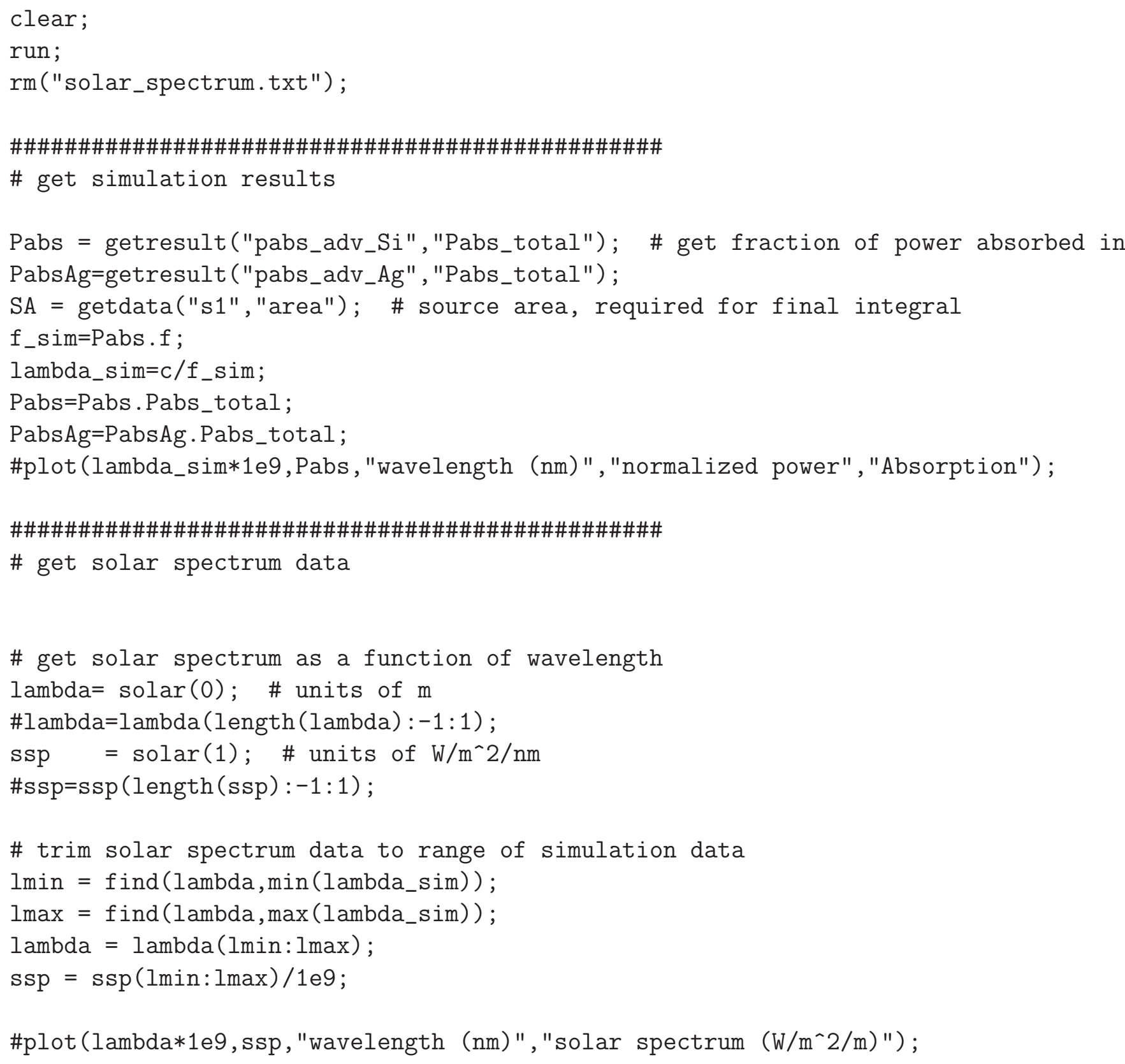









\section{A.3 I-V curve Calculation (DEVICE)}

This script performs an electrical simulation using the finite-element method to determine the relevant solar cell parameters using electron transport equations. The simulation takes into account surface and bulk recombination as well as electron and hole velocities in the amorphous silicon material. We have used commonly cited values for a-Si as follows:

- $e^{-}$mobility $10 \mathrm{~cm}^{2} / V-s$

- $h^{+}$mobility $2 \mathrm{~cm}^{2} / V-s$

- SRH recombination: $e^{-}$lifetime $5.6 \mu s, h^{+}$lifetime $.4 \mu s$

- Radiative and Auger recombination included as well

- Surface recombination velocities $(\mathrm{cm} / \mathrm{s}): \mathrm{Al} 1 e^{+7}, \mathrm{Ag} 1 e^{+5}, \mathrm{ITO} 1 e^{+2}, \mathrm{SiO}_{2}$ $1 e^{+2}$

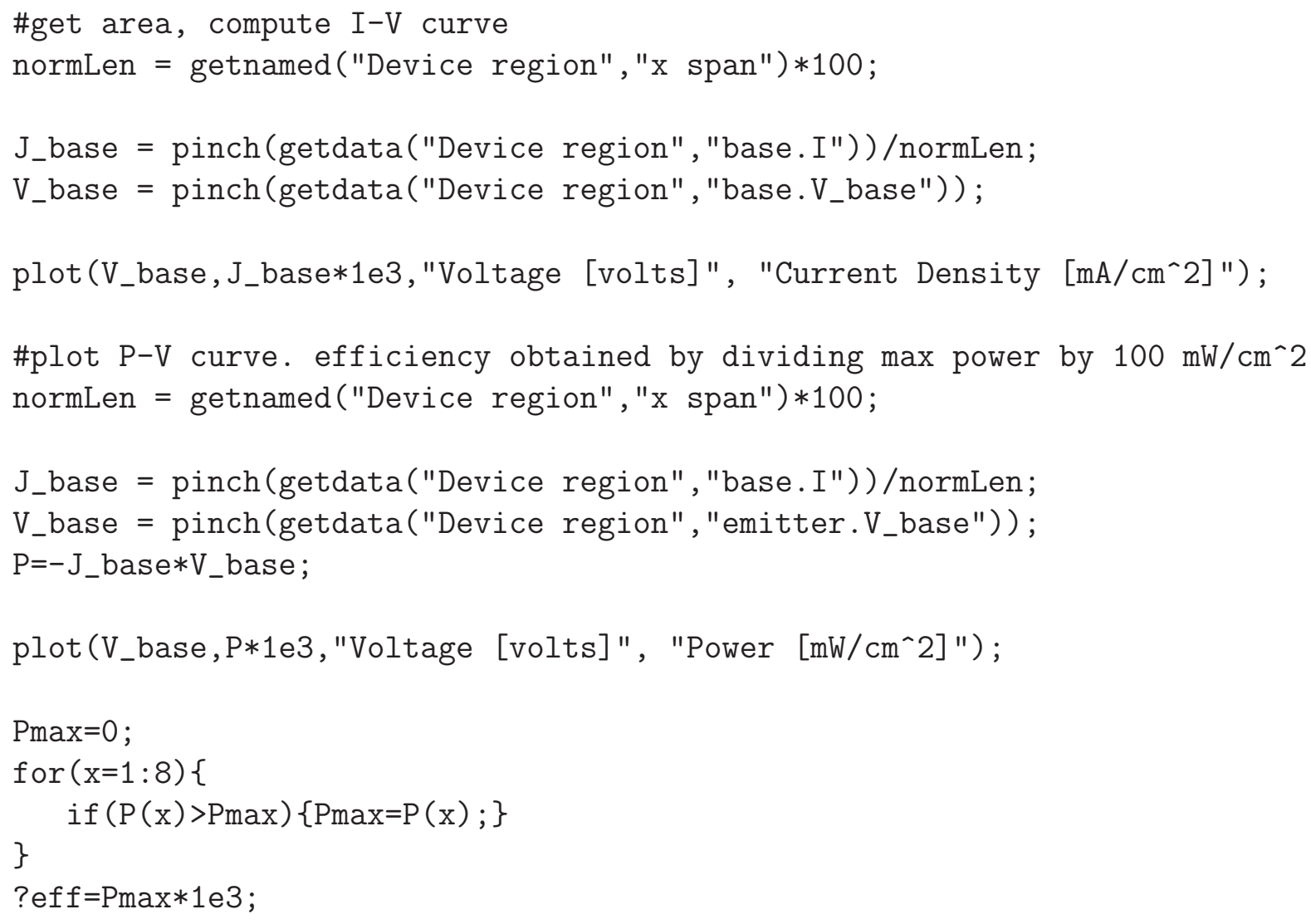




\section{Appendix B}

\section{Fabrication Process Flow Sheet}

Below is a brief outline of the procedure followed for fabricating the test cells with nanocubes on a top layer of 20nm ITO. The layout is shown in Fig. B.1. Some important fabrication notes are also included here.

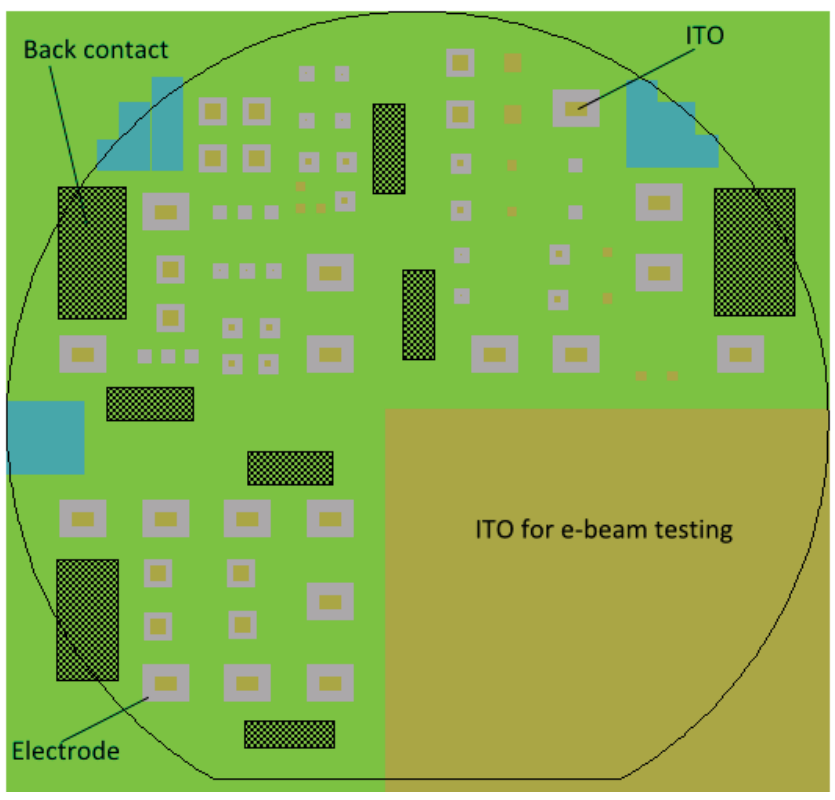

Figure B.1: L-Edit wafer mask layout 
The following is a step by step process for fabricating the thin-film solar cells on a 2" c-Si wafer:

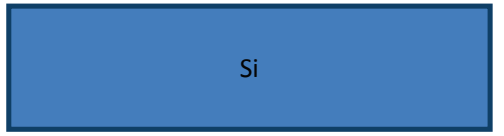

1. n-type Si wafer with RCA clean

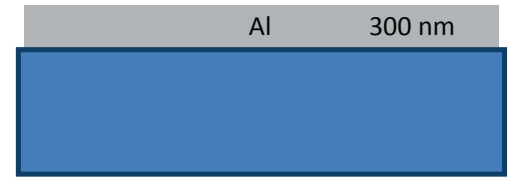

2. Deposit $300 \mathrm{~nm} \mathrm{Al}$ as back contact: Balzers evaporator

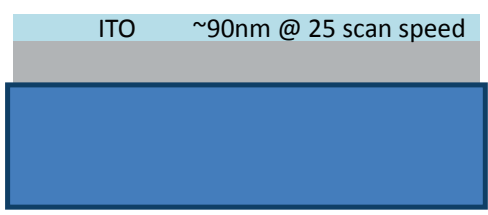

3. Deposit 100nm ITO as recombination barrier: Semicore PVD

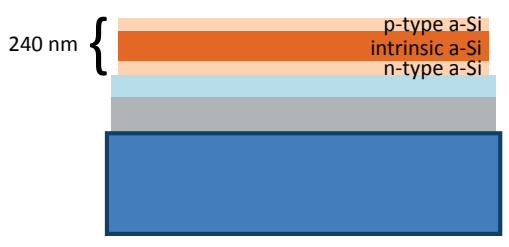

4. Deposit 20nm n-type, $210 \mathrm{~nm}$ i-type, $15 \mathrm{~nm}$ ptype nc-Si: PECVD

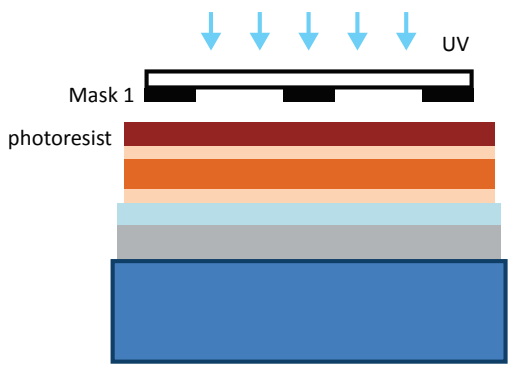

5. Liquid HMDS coat then photoresist (LOR10B and S1811) deposition, expose $(50 s)$



6. Develop photoresist

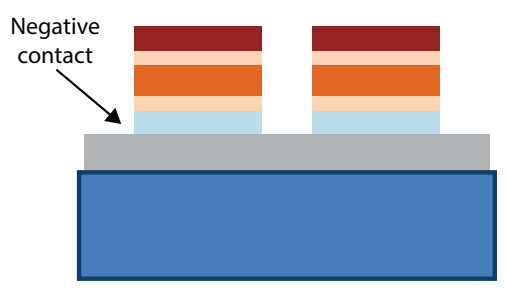

7. Etch nc-Si for back contacts: Technics etcher

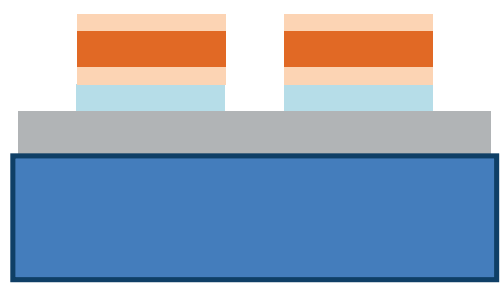

8. Remove resist 


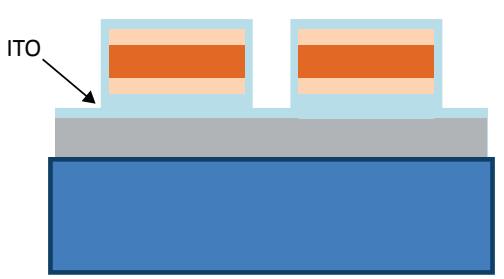

9. Deposit 20nm ITO: Semicore PVD

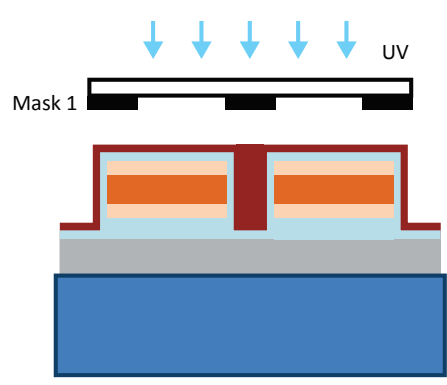

10. Liquid HMDS then positive photoresist (LOR10B and S1811) spin coat to define active cell areas

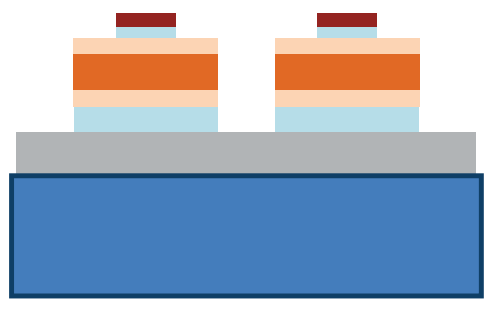

11. Develop photoresist

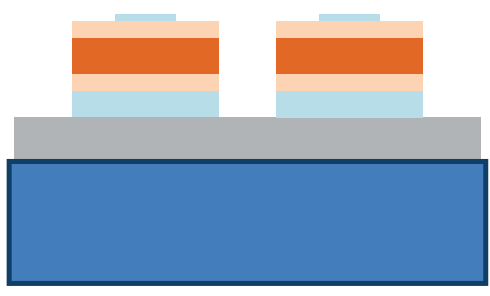

12. Etch ITO in Aqua regia $(20 s)$ then remove resist in acetone plus Microposit Remover 1165

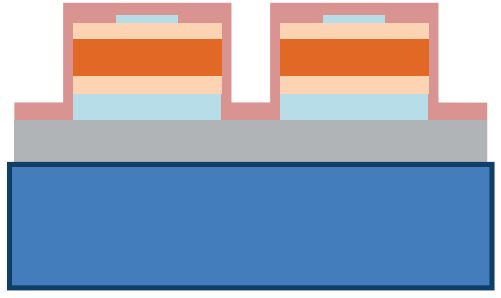

13. Spin coat $180 \mathrm{~nm}$ PMMA for e-beam, write nanocube array then deposit $80 \mathrm{~nm} \mathrm{Al}$ and metal lift-off



14. Spin coat photoresist (LOR10B and S1811) for top contact mask, expose

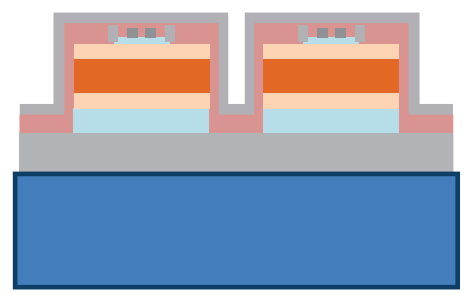

15. Develop resist then deposit $30 \mathrm{~nm}$ $\mathrm{Ti} / 300 \mathrm{~nm} \mathrm{Ag}$ 


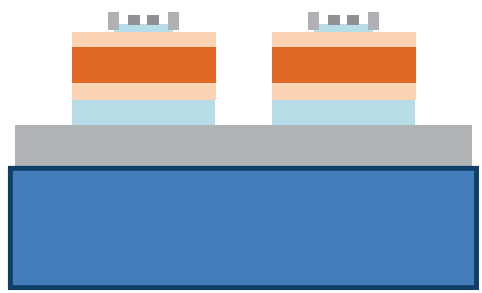

16. Lift-off resist 